\title{
On the Equations Defining Abelian Varieties. I
}

\section{Citation}

Mumford, David B. 1966. On the equations defining abelian varieties. I. Inventiones

Mathematicae 1(4): 287-354.

\section{Published Version}

doi:10.1007/BF01389737

\section{Permanent link}

http://nrs.harvard.edu/urn-3:HUL.InstRepos:3597241

\section{Terms of Use}

This article was downloaded from Harvard University's DASH repository, and is made available under the terms and conditions applicable to Other Posted Material, as set forth at http:// nrs.harvard.edu/urn-3:HUL.InstRepos:dash.current.terms-of-use\#LAA

\section{Share Your Story}

The Harvard community has made this article openly available.

Please share how this access benefits you. Submit a story.

Accessibility 


\section{On the Equations Defining Abelian Varieties. I*}

D. MuMford (Cambridge, Mass.)

\section{Contents}

$\S 1$. The Basic Groups Acting on Linear Systems . . . . . . . . . . . . . . 288

§. Symmetric Invertible Sheaves . . . . . . . . . . . . . . . . 303

§3. The Addition Formula . . . . . . . . . . . . . . . . . . . . 320

§. Structure of the Homogeneous Coordinate Ring . . . . . . . . . . . 336

§5. Examples . . . . . . . . . . . . . . . . . . . . . 349

References . . . . . . . . . . . . . . . . . 354

My aim is to set up a purely algebraic theory of theta-functions. Actually, since my methods are algebraic and not analytic, the functions themselves will not dominate the picture - although they are there. The basic idea is to construct canonical bases of all linear systems on all abelian varieties. The result is that one gets a very complete description first of the homogeneous coordinate ring of a single abelian variety, and second of the moduli space of all abelian varieties. We shall obtain explicit generators and relations for this moduli space. The homogeneous coordinate rings of abelian varieties are very remarkable rings. Although the abelian variety is a commutative group, these rings are acted upon (with some restriction on degrees) by a 2-step nilpotent group. Unlike the affine coordinate rings of linear algebraic groups, they are not Hopf algebras. Their structure is dominated by a symmetry of a higher order embodied in the theta relation of Riemann (a quartic relation). One might say that this is the only class of rings not "essentially" isomorphic to polynomial rings which we can describe so closely.

There are several interesting topics which I have not gone into in this paper, but which can be investigated in the same spirit: for example, the extension to inseparably polarized abelian varieties; a discussion of the transformation theory of theta-functions, especially in connection with the tower of moduli schemes; a discussion of the various standard models of the irreducible representation space for the Heisenberg commutation relations [and the adelic generalization] and the various ways in which RIEMANN's theta-function can be singled out in each of them; an analysis of special theta-functions and special abelian varieties; an analysis of degenerate theta functions and SATAKE's compactification; a tie-up between the global theory of moduli that we give, and the

\footnotetext{
* This work was partially supported by NSF grant GP 3512 and a grant from the Sloan Foundation.
}

20 Invent. math., Bd. 1 
infinitesimal theory of KoDAIRA-SPENCER-GROTHENDIECK. All of these look like very fruitful topics. Incidentally, in $\S 6$ there is a very annoying 8 in the main result which by all rights ought to be replaced by 4 [but I nearly despaired of getting the 8 in the course of proving what I named the "Hardest lemma" in §6].

This paper is heavily indebted to the influence and ideas of BAILY, CARTIER, IguSA, MAYer, Siegel, and Weil. As an algebraist, I was naturally not attracted to anything called a function, and it was only because these six people all realized so clearly the significance of thetafunctions that the idea got across to me. More than that, many of the key ideas are due to these people and especially to IGUSA: the reader is referred especially to the important papers $[1,2,6,11$ and 12]. In particular, the beautiful and far-reaching fact that the theta-null values give almost exactly the moduli space of a carefully chosen level is IGUSA's idea.

In most of this paper, we will work over a fixed algebraically closed ground field $k$. At first, $k$ will have any characteristic; later we will exclude characteristic 2 . Of course we use the language of schemes. Also, if $S$ is a finite set, \# $(S)$ denotes the cardinality of $S$.

A word of warning - and apology. There are several thousand formulas in this paper which allow one or more "sign-like ambiguities": i.e., alternate and symmetric but non-equivalent reformulations. These occur in definitions and theorems. I have made a superhuman effort to achieve consistency and even to make correct statements: but I still cannot guarantee the result.

\section{§1. The Basic Groups Acting on Linear Systems}

$X$ will denote an abelian variety for all of this paper. All varieties that we will talk about will be abelian varieties; this will be mentioned from time to time but not invariably. When we talk of an abelian variety $X$, we always assume that a definite identity point $e \in X$ has been chosen: hence a definite group law on $X$ has been chosen. Moreover, the endomorphism of an abelian variety given by multiplication by $n$ will be denoted by $n \delta$. The inverse $-\delta$ will be denoted $t$. The kernel of $n \delta$ will be denoted $X_{n}$.

Definition. If $L$ is an invertible sheaf on $X$, then $H(L)$ is the subgroup of closed points $x \in X$ such that if $T_{x}: X \rightarrow X$ denotes translation by $x$, then $T_{x}^{*} L \cong L$.

We recall the basic facts about invertible sheaves on an abelian variety, and their sections (cf. [9], Ch. 6, § 2):

(I) $L$ is ample if and only if $H(L)$ is finite and $\Gamma\left(X, L^{n}\right)$ is not (0) for all $n>0$. 
(II) If $L$ is ample and $\operatorname{dim} X=g$, then there is a positive integer $d$ such that

$$
\begin{aligned}
& \operatorname{dim} H^{0}\left(X, L^{n}\right)=d \cdot n^{g}, \quad \text { all } n \geqq 1 \\
& \operatorname{dim} H^{i}\left(X, L^{n}\right)=0, \quad \text { all } \quad n \geqq 1, i \geqq 1 \text {. }
\end{aligned}
$$

(III) The integer $d$ of (II) is called the degree of $L$, and if $D$ is a divisor on $X$ defining $L$, i.e., $L \cong \boldsymbol{o}_{X}(D)$, then

$$
\left(D^{g}\right)=d \cdot g \text { ! }
$$

(IV) Let $\hat{X}$ be the dual of $X$. Let $\Lambda(L): X \rightarrow \hat{X}$ be the usual homomorphism associated to $L$, i.e., if $x \in X_{k}$ then $\Lambda(L)(x)$ is the point of $\hat{X}$ corresponding to the sheaf $T_{x}^{*}(L) \otimes L^{-1}$ on $X$. Then

$$
\text { degree } \Lambda(L)=d^{2} \text {. }
$$

(V) Let $L^{\prime}=l^{*} L$ be the reflection of $L$ in the origin. Then for all integers $n$,

$$
(n \delta)^{*} L \cong(L)^{\frac{n^{2}+n}{2}} \otimes\left(L^{\prime}\right)^{\frac{n^{2}-n}{2}} .
$$

In this paper we shall be exclusively interested in invertible sheaves $L$ such that

a) $L$ is ample.

b) If $p=\operatorname{char}(k), p \nmid$ degree $(L)$.

We shall refer to such sheaves as ample sheaves of separable type. Note that for such sheaves, by (IV), $\operatorname{char}(k) \chi$ degree $\Lambda(L)$, so $\Lambda(L)$ is separable. Since, by definition $H(L)$ is the kernel of $\Lambda(L)$, it follows that:

$$
d^{2}=\text { cardinality } H(L) \text {. }
$$

At this point, I can define the most central concept of the entire development:

Definition. Let $L$ be an ample invertible sheaf of separable type. Then $\mathscr{G}(L)$ is the set of pairs $(x, \varphi)$, where $x$ is a closed point of $X$ and $\varphi$ is an isomorphism:

$$
L \underset{\varphi}{\stackrel{\sim}{\longrightarrow}} T_{x}^{*} L .
$$

First of all: $\mathscr{G}(L)$ is a group. Let $(x, \varphi),(y, \psi) \in \mathscr{G}(L)$. Then the composition $T_{x}^{*} \psi \circ \varphi$ :

$$
L \stackrel{\varphi}{\longrightarrow} T_{x}^{*} L \stackrel{T_{x}^{*} \psi}{\longrightarrow} T_{x}^{*}\left(T_{y}^{*} L\right)=T_{x+y}^{*} L
$$

is an isomorphism of $L$ and $T_{x+y}^{*} L$. Define

$$
(y, \psi) \circ(x, \varphi)=\left(x+y, T_{x}^{*} \psi \circ \varphi\right) .
$$

20* 
One checks immediately that this makes $\mathscr{G}(L)$ into a group. Secondly, the map taking $(x, \varphi)$ to $x$ puts $\mathscr{G}(L)$ into the exact sequence:

$$
0 \rightarrow k^{*} \rightarrow \mathscr{G}(L) \rightarrow H(L) \rightarrow 0 \text {. }
$$

Here $\mathscr{G}(L) \rightarrow H(L)$ is surjective by the very definition of $H(L)$, and the kernel is the group of isomorphisms of $L$ with itself: i.e., of multiplications by non-zero constants in the ground field $k$.

Our first objective in this section is to give a complete structure theorem for $H(L)$ and $\mathscr{G}(L)$ vis-a-vis this exact sequence. To this end, we must first examine the situation:

$$
\begin{aligned}
& X \stackrel{\pi}{\longrightarrow} Y \\
& L \stackrel{\alpha}{\longrightarrow} M .
\end{aligned}
$$

Here $\pi$ stands for a separable isogeny of the abelian varieties $X$ and $Y$, $M$ stands for an invertible sheaf on $Y$ and $\alpha$ stands for an isomorphism:

$$
\alpha: \quad \pi^{*} M \stackrel{\sim}{\longrightarrow} L .
$$

Let $K$ be the kernel of $\pi: K$ is a finite subgroup of closed points of $X$. Moreover, if $x \in K$, then

$$
L \underset{\alpha}{\stackrel{\sim}{\sim}} \pi^{*} M=\left(\pi \circ T_{x}\right)^{*} M=T_{x}^{*}\left(\pi^{*} M\right) \underset{T_{x}^{*}(\alpha)}{\sim} T_{x}^{*} L
$$

defines an isomorphism of $T_{x}^{*} L$ and $L$, so $x \in H(L)$. Thus $K \subset H(L)$. But let the reader beware - a key point is that if $K \subset H(L)$ is an arbitrary subgroup, then there does not necessarily exist an invertible sheaf $M$ on $Y=X / K$ such that $\pi^{*} M \cong L$. In fact, as GROTHENDIECK's theory of descent teaches us, it is absolutely essential to consider the pair

$$
\left(x, T_{x}^{*}(\alpha) \circ \alpha^{-1}\right)
$$

as an element of $\mathscr{G}(L)$. If the set of these is denoted $\tilde{K}$, then one checks immediately that they form a subgroup of $\mathscr{G}(L)$. Namely:

$$
\begin{aligned}
& \left(x, T_{x}^{*}(\alpha) \circ \alpha^{-1}\right) \circ\left(y, T_{y}^{*}(\alpha) \circ \alpha^{-1}\right) \\
& \quad=\left(x+y, T_{y}^{*}\left\{T_{x}^{*}(\alpha) \circ \alpha^{-1}\right\} \circ\left\{T_{y}^{*}(\alpha) \circ \alpha^{-1}\right\}\right) \\
& \quad=\left(x+y, T_{x+y}^{*}(\alpha) \circ \alpha^{-1}\right) .
\end{aligned}
$$

In other words, we have split the extension $\mathscr{G}(L) \rightarrow H(L)$ over $K$ :

$$
\begin{gathered}
\mathscr{G}(L) \longrightarrow H(L) \longrightarrow 0 \\
\cup \\
\tilde{K} \stackrel{\cup}{\longrightarrow} K .
\end{gathered}
$$

Conversely, given a subgroup $K \subset H(L)$, and a lifting $K$ of $K$ into $\mathscr{G}(L)$, we have exactly the situation referred to by GROTHENDIECK as descent 
data for $L$ with respect to the morphism

$$
X \stackrel{\pi}{\longrightarrow} Y=X / K \text {. }
$$

His main theorem (Theorem $1.1, \S 8,[3]$ ) says that there is a $1-1$ correspondence between the set of invertible sheaves $M$ on $Y$ and isomorphisms $\alpha: \pi^{*} M \stackrel{\sim}{\longrightarrow} L$ and the set of descent data for $L$ with respect to $\pi$. We make the definition:

Definition. A level subgroup $\tilde{K}$ of $\mathscr{G}(L)$ is a subgroup such that $k^{*} \cap \widetilde{K}=(0)$, i.e., $\widetilde{K}$ is isomorphic to its image $K$ in $H(L)$.

Thus, our conclusion may be rephrased:

Proposition 1. There is a 1-1 correspondence between level subgroups $\tilde{K}$ in $\mathscr{G}(L)$ and pairs $(\pi, \alpha)$ as in (*) above.

What happens to the degrees in this situation? Note first that $M$ is certainly ample (cf. Theorem 2.6.2, Ch. 3, [5]). Say

$$
\begin{aligned}
& d=\text { degree } L \\
& r=\operatorname{degree}(\pi)=\text { cardinality }(K) .
\end{aligned}
$$

Then if $E$ is a Cartier divisor on $Y$ representing $M, \pi^{-1}(E)$ is a Cartier divisor on $X$ representing $L$ and

$$
\begin{aligned}
d \cdot g ! & =\left(\pi^{-1}(E)^{g}\right) \\
& =\pi^{-1}\left(E^{g}\right) \\
& =r\left(E^{g}\right) \\
& =r \cdot \text { degree } M \cdot g ! .
\end{aligned}
$$

In particular, char $(k) \chi$ degree $(M)$, so $M$ is of separable type too. And degree $(M)=d / r$. Moreover, it is possible to relate $\mathscr{G}(M)$ and $\mathscr{G}(L)$. If the map assigning to $X$ and $L$ the group $\mathscr{G}(L)$ was part of a functor, we would expect there to exist a homomorphism from $\mathscr{G}(L)$ to $\mathscr{G}(M)$ or vice versa. Actually, the situation is more complicated than that. In fact:

Proposition 2. Given the situation (*) above, let $K=\operatorname{ker}(\pi)$ and let $\tilde{K}$ be the corresponding level subgroup over $K$.

i) $\pi^{-1}[H(M)] \subset H(L)$.

ii) $\left\{\begin{array}{l}\text { centralizer } \\ \text { of } \tilde{K} \text { in } \mathscr{G}(L)\end{array}\right\}=\left\{z \in \mathscr{G}(L) \mid \begin{array}{l}\text { the image of } z \text { in } H(L) \\ \text { is in } \pi^{-1}[H(M)]\end{array}\right\}$.

Call this group $\mathscr{G}(L)^{*}$.

iii) $\mathscr{G}(M) \underset{\text { canonically }}{\cong} \mathscr{G}(L)^{*} / \tilde{K}$.

(In particular, $\mathscr{G}(M)$ is a quotient of a subgroup of $\mathscr{G}(L)$ ). 
Proof. Suppose $y \in Y_{k}$ and $M \cong T_{y}^{*} M$. Then if $x \in X_{k}$ and $\pi(x)=y$,

$$
\begin{aligned}
T_{x}^{*} L & \cong T_{x}^{*} \pi^{*} M \\
& \cong \pi^{*} T_{y}^{*} M \\
& \cong \pi^{*} M \\
& \cong L .
\end{aligned}
$$

This proves (i). More precisely, if $(y, \psi) \in \mathscr{G}(M)$, we see that

$$
\left(x, T_{x}^{*}(\alpha) \circ \pi^{*} \psi \circ \alpha^{-1}\right) \in \mathscr{G}(L) .
$$

But $x$ determines $y$, and the homomorphism $T_{x}^{*}(\alpha) \circ \pi^{*} \psi \circ \alpha^{-1}$ determines $\psi$. Therefore, this relation between elements of $\mathscr{G}(M)$ and $\mathscr{G}(L)$ is actually a map:

$$
\mathscr{G}(L) \supset\{(x, \varphi) \in \mathscr{G}(L) \mid \pi(x) \in H(M)\} \rightarrow \mathscr{G}(M) .
$$

Call this subgroup $\mathscr{G}(L)^{*}$. This map is readily checked to be a homomorphism. Moreover, every $y \in H(M)$ is of the form $\pi(x)$, some $x \in H(L)$; and if some element $\left(y, \varphi_{1}\right)$ is in its image, then all other elements $\left(y, \varphi_{2}\right)$ are in the image since $\varphi_{2}$ is always a scalar multiple of $\varphi_{1}$. Therefore $\mathscr{G}(M)$ is a quotient of $\mathscr{G}(L)^{*}$. One checks easily that the kernel is $\tilde{K}$, and this proves (iii). Finally, (ii) is a consequence of descent theory. According to this theory, suppose $M_{1}$ and $M_{2}$ are obtained by descending (over a faithfully flat morphism $\pi$ ) sheaves $L_{1}=\pi^{*} M_{1}$ and $L_{2}=\pi^{*} M_{2}$. Then the set of homomorphisms from $M_{1}$ to $M_{2}$ is the same as the set of homomorphisms from $L_{1}$ to $L_{2}$ that "commute" with the descent data. In our case, $M$ is obtained by descending $L$ via the identifications:

$$
L \stackrel{\varphi}{\sim} T_{w}^{*} L, \text { all }(w, \varphi) \in \tilde{K},
$$

and if $y=\pi(x) \in Y_{k}$, then $T_{y}^{*} M$ is obtained by descending $T_{x}^{*} L$ via the identifications

$$
T_{x}^{*} L \stackrel{T_{x}^{*} \varphi}{\sim} T_{x+w}^{*} L, \quad \text { all }(w, \varphi) \in \tilde{K} .
$$

Therefore, an isomorphism $\psi: L \rightarrow T_{x}^{*} L$ "descends" if and only if the diagram:

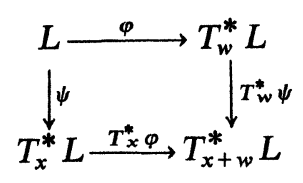

commutes, for all $(w, \varphi) \in \tilde{K}$. On the one hand, this means exactly that $(x, \psi)$ is in the centralizer of $\tilde{K}$; on the other hand, $\psi$ descends to an isomorphism of $M$ and $T_{y}^{*} M$ if and only if $y \in H(M)$, i.e., $(x, \psi) \in \mathscr{G}(L)^{*}$. This proves (ii). Q.E.D. 
Now consider the extension $\mathscr{G}(L)$ of $H(L)$ by $k^{*}$. Note first that $k^{*}$ is contained in the center of $\mathscr{G}(L)$. This follows immediately from the definition of multiplication in $\mathscr{G}(L)$. The extension defines the following invariant:

$$
\left\{\begin{array}{l}
\text { Given } x, y \in H(L), \text { let } \tilde{x}, \tilde{y} \in \mathscr{G}(L) \text { lie over } x, y . \\
\text { Set } e^{L}(x, y)=\tilde{x} \cdot \tilde{y} \cdot \tilde{x}^{-1} \cdot \tilde{y}^{-1}
\end{array}\right.
$$

It is immediate that this is well-defined, that $e^{L}(x, y)$ is an element of $k^{*}$, and that $e^{L}$ is a skew-symmetric bilinear pairing from $H(L)$ to $k^{*}$ : i.e.,

(a) $e^{L}\left(x_{1}+x_{2}, y\right)=e^{L}\left(x_{1}, y\right) \cdot e^{L}\left(x_{2}, y\right)$,

(b) $e^{L}(x, x)=1$, hence $e^{L}(x, y)=e^{L}(y, x)^{-1}$.

Moreover, because $k^{*}$ is divisible, for all subgroups $K \subset H(L)$, there exists a level subgroup $\tilde{K}$ over $K$ if and only if $e^{L} \equiv 1$ on $K$. To see this, first start with a single element $x \in K$. Let $\tilde{x}^{\prime} \in \mathscr{G}(L)$ lie over $x$. If $l$ is the order of $x$, then $\left(\tilde{x}^{\prime}\right)^{l}$ must be in $k^{*}$. Let $\alpha \in k^{*}$ be an $l$-th root of $\left(\tilde{x}^{\prime}\right)^{l}$, and let $\tilde{x}=\tilde{x}^{\prime} \cdot \alpha^{-1}$. Then $\tilde{x}$ lies over $x$ and also has order $l$. Now writing $K$ as a direct sum of cyclic groups, and lifting its generators $x_{i}$ by this procedure, we see that since $e^{L} \equiv 1$ on $K$, the elements $\tilde{x}_{i}$ generate a level subgroup $\tilde{K}$ over $K$. Conversely, if $\tilde{K}$ exists, then $\tilde{K}$ is commutative, so $e^{L} \equiv 1$ on $K$.

The following conditions are readily seen to be equivalent:

i) $k^{*}=$ center $[\mathscr{G}(L)]$.

ii) $e^{L}$ is non-degenerate: i.e., $\forall x \in H(L)$, there exists a $y \in H(L)$ such that $e^{L}(x, y) \neq 1$,

iii) there are subgroups $K_{1}, K_{2}$ in $H(L)$ such that $H(L)=K_{1} \oplus K_{2}$, $e^{L}(x, y)=1$ if $x, y \in K_{1}$ or if $x, y \in K_{2}$ and $e^{L}$ is a non-degenerate pairing of $K_{1}$ and $K_{2}-$ i.e., makes

$$
K_{2} \cong \operatorname{Hom}\left(K_{1}, k^{*}\right) \text {. }
$$

In fact, i) and ii) are trivially equivalent and are implied by iii). To go from ii) to iii), one just mimics the standard procedure for putting skew-symmetric pairings in canonical form.

Theorem 1. The above condition on $\mathscr{G}(L)$ is, in fact, satisfied.

Proof. Let $\tilde{K}$ be a maximal level subgroup in $\mathscr{G}(L)$ and let $K$ be its image in $H(L)$. Then $K$ is a maximal subgroup of $H(L)$ on which $e^{L} \equiv 1$, and the centralizer of $\tilde{K}$ in $\mathscr{G}(L)$ is just $k^{*} \cdot \tilde{K}$. Let $Y=X \mid K$ and let $M$ be the sheaf on $Y$ obtained by descending $L$. Then according to our description of $H(M)$ given above, $H(M)=\{0\}$ : in other words, $M$ has degree 1 . But since degree $M=d / r$, where $r=$ cardinality of $K$, this means that the cardinality of $K$ is $d$. Now suppose $H_{0} \subset H(L)$ is the 
degenerate space of $e^{L}$ - the set of elements $x \in H(L)$ such that $e^{L}(x, y)=1$, all $y \in H(L)$. Then $H(L) / H_{0}$ satisfies conditions ii) and iii); therefore

$$
\#\left(H(L) / H_{0}\right)=l^{2}
$$

and there exist maximal subgroups $K^{\prime} \subset H(L) / H_{0}$ on which $e^{L} \equiv 1$ of cardinality $l$. Now if $K$ is the inverse image of $K^{\prime}$ in $H(L)$, we see:

$$
\begin{aligned}
d^{2} & =\#(K)^{2} \\
& =\#\left(H_{0}\right)^{2} \cdot l^{2} \\
& =\#\left(H_{0}\right)^{2} \cdot \#\left(H(L) / H_{0}\right) \\
& =\#\left(H_{0}\right) \cdot \#(H(L)) \\
& =\#\left(H_{0}\right) \cdot d^{2} .
\end{aligned}
$$

Therefore $H_{0}=(0)$. Q.E.D.

Definition. Let $L$ be an ample invertible sheaf of separable type. By Theorem 1, the elementary divisors on $H(L)$ occur in pairs. Let

$$
\delta=\left(d_{1}, d_{2}, \ldots, d_{k}\right)
$$

be the sequence of positive integers such that $d_{i+1} \mid d_{i}, d_{i}>1$, and such that

$$
\left(d_{1}, d_{1}, d_{2}, d_{2}, \ldots, d_{k}, d_{k}\right)
$$

are the elementary divisors on $H(L)$. Then $L$ will be said to be of type $\delta$.

Note that char $(k) \nmid d_{1}$ in this sequence. Now reversing the process:

Definition. Let $\delta=\left(d_{1}, \ldots, d_{k}\right)$ be a sequence as above. Let

$$
\begin{aligned}
& K(\delta)=\bigoplus_{i=1}^{k} \boldsymbol{Z} / d_{i} \boldsymbol{Z} \\
& \widehat{K(\delta)}=\operatorname{Hom}\left(K(\delta), k^{*}\right) \\
& H(\delta)=K(\delta) \oplus \widehat{K(\delta)} .
\end{aligned}
$$

Let $\mathscr{G}(\delta)$, as a set, be the product

$$
k^{*} \times K(\delta) \times \widehat{K(\delta)} .
$$

Define a group law on $\mathscr{G}(\delta)$ via

$$
(\alpha, x, l) \cdot\left(\alpha^{\prime}, x^{\prime}, l^{\prime}\right)=\left(\alpha \cdot \alpha^{\prime} \cdot l^{\prime}(x), x+x^{\prime}, l+l^{\prime}\right) .
$$

Corollary of Th. 1. If $L$ is of type $\delta$, then the sequence

$$
0 \rightarrow k^{*} \rightarrow \mathscr{G}(L) \rightarrow H(L) \rightarrow 0
$$


is isomorphic to the sequence:

$$
0 \rightarrow k^{*} \rightarrow \mathscr{G}(\delta) \rightarrow H(\delta) \rightarrow 0 .
$$

Proof. As in condition (iii) preceding the theorem, let $H(L)=K_{1} \oplus K_{2}$ and let $\tilde{K}_{i}$ be a level subgroup over $K_{i}$. Choose any isomorphism $\alpha$ between $K_{1}$ and $K(\delta)$, and then map $K_{2}$ onto $K(\delta)$ via

$$
\beta: \quad K_{2} \cong \operatorname{via} e
$$

If we require that $(1, x, 0)$ correspond to the point of $\tilde{K}_{1}$ over $\alpha^{-1}(x) \in K_{1}$, and $(1,0, l)$ correspond to the point $\tilde{K}_{2}$ over $\beta^{-1}(l) \in K_{2}$, then this determines an isomorphism of $\mathscr{G}(\delta)$ and $\mathscr{G}(L)$. Q.E.D.

So far, we have considered $\mathscr{G}(L)$ only as a group in its own right, and as the set of all possible descent data for $L$ with respect to isogenies. What makes this game more exciting however is that $\mathscr{G}(L)$ acts on $\Gamma(X, L)$ :

Definition. Let $z=(x, \varphi) \in \mathscr{G}(L)$. Then define $U_{z}: \Gamma(X, L) \rightarrow \Gamma(X, L)$ by

$$
U_{z}(s)=T_{-x}^{*}(\varphi(s))
$$

for all $s \in \Gamma(X, L)$ [i.e., $\varphi(s)$ is a section of $T_{x}^{*} L$ and $T_{-x}^{*}(\varphi(s))$ is a section of $\left.L=T_{-x}^{*}\left(T_{x}^{*} L\right)\right]$.

This is an action of the group $\mathscr{G}(L)$ since if $z=(x, \varphi), w=(y, \psi)$, then

$$
\begin{aligned}
U_{w}\left(U_{z}(s)\right) & =T_{-y}^{*}\left\{\psi\left(T_{-x}^{*}(\varphi s)\right)\right\} \\
& =T_{-x-y}^{*}\left[T_{x}^{*}\left(\psi\left(T_{-x}^{*}(\varphi s)\right)\right)\right] \\
& =T_{-x-y}^{*}\left[T_{x}^{*}(\psi)(\varphi s)\right] \\
& =U_{\left(x+y, T_{x}^{*}(\psi) \circ \varphi\right)}(s) .
\end{aligned}
$$

Also, the center $k^{*}$ of $\mathscr{G}(L)$ acts on $\Gamma(X, L)$ by its natural character: i.e., $\alpha \in k^{*}$ acts on $\Gamma(X, L)$ as multiplication by $\alpha$. Such representations are rather limited:

Proposition 3. $\mathscr{G}(\delta)$ has a unique irreducible representation in which $k^{*}$ acts by its natural character. Suppose that this representation is denoted by $V(\delta)$. Then if $V$ is any representation of $\mathscr{G}(\delta)$ in which $k^{*}$ acts in this way, $V$ is isomorphic to the direct sum of $V(\delta)$ with itself r-times for some $r$. Moreover, if $\tilde{K} \subset \mathscr{G}(\delta)$ is any maximal level subgroup,

$$
r=\operatorname{dim}_{k}\left(V^{\tilde{K}}\right) \text {. }
$$

(Here $V^{\tilde{K}}$ is the subspace of $V$ of $\tilde{K}$-invariants.) 
We give a complete proof here since it is quite instructive. The result is an exact analog of a very general theorem of MACKEY [8].

Proof. Let $V$ be an irreducible representation of $\mathscr{G}(\delta)$. Pick a maximal level subgroup $\tilde{K}$. Then since $\tilde{K}$ is commutative and char $(k) \ngtr$ cardinality $\tilde{K}$, the action of $\tilde{K}$ can be diagonalized: i.e.,

$$
V=\underset{\chi \in \operatorname{Hom}\left(\tilde{K}, k^{*}\right)}{\oplus} V_{\chi},
$$

where $V_{\chi}$ is the eigenspace with weight $\chi$. Now let $y \in \mathscr{G}(\delta)$. Then there is a unique character $\chi^{y}$ of $\tilde{K}$ defined by:

$$
y^{-1} \cdot z \cdot y=\chi^{y}(z) \cdot z, \text { all } z \in \tilde{K},
$$

and if $s \in V_{\chi_{0}}$, then one checks easily that $U_{y}(s) \in V_{\chi_{0}+\chi^{y}}$ (here $U_{y}$ is the given representation). Moreover, note that the mapping $y \rightarrow \chi^{y}$ defines an isomorphism $\gamma$ :

$$
\mathscr{G}(\delta) / k^{*} \cdot \tilde{K} \underset{\gamma}{\stackrel{\sim}{\longrightarrow}} \operatorname{Hom}\left(\tilde{K}, k^{*}\right) .
$$

Therefore: (a) if $V_{\chi} \neq(0)$ for one $\chi$, then $V_{\chi} \neq 0$ for all $\chi$. (b) If $s \in V_{\chi_{0}}$, then the elements $U_{y}(s)$ span a subspace $W$ of $V$ such that $W \cap V_{\chi}$ is one-dimensional for all $\chi$. It follows that if $V$ is irreducible, $\operatorname{dim} V_{\chi}=1$ for all $\chi$. Now choose an arbitrary section $\sigma$ :

$$
\mathscr{G}(\delta) \stackrel{\sigma}{\longrightarrow} \mathscr{G}(\delta) / k^{*} \cdot \tilde{K}
$$

Moreover, choose a non-zero element $s(0) \in V_{0}$. Then for all $\chi \in \operatorname{Hom}\left(\tilde{K}, k^{*}\right)$, set $s(\chi)=U_{\sigma\left(\gamma^{-1}(\chi)\right)}(s(0))$. Clearly $\{s(\chi)\}$ form a basis of $V$. It is easily checked that the matrices giving the representation of $\mathscr{G}(\delta)$ in terms of this basis depend only on the section $\sigma$. Therefore, all irreducible representations $V$ are isomorphic. The last two assertions about a general representation $V$ follow immediately from the complete reducibility of such representations. In fact, let $m=$ order of $H(\delta)$. Then the set of elements $x \in \mathscr{G}(\delta)$ such that $x^{m}=1$ form a finite subgroup $\mathscr{G}(\delta)^{\prime} \subset \mathscr{G}(\delta)$. Since $k^{*} \mathscr{G}(\delta)^{\prime}=\mathscr{G}(\delta)$, a representation $V$ of $\mathscr{G}(\delta)$ in which $k^{*}$ acts by its natural character is completely reducible if and only if it is completely reducible as a representation of $\mathscr{G}(L)^{\prime}$. But char $(k) \chi$ order $\left(\mathscr{G}(L)^{\prime}\right)$, so representations of $\mathscr{G}(L)^{\prime}$ are completely reducible.

$$
\operatorname{dim}\left(V^{\tilde{K}}\right)=\operatorname{dim} V_{0}=1
$$

if $V$ is irreducible. Q.E.D.

There are several natural ways to write down this unique irreducible representation explicitly. These explicit representations and the transformations between them have been closely studied by WEIL [12]. The simplest is: 
Definition. Let $V(\delta)$ be the vector space of functions $f$ on $K(\delta)$ with values in $k$. Let $\mathscr{G}(\delta)$ act on this by:

$$
\left(U_{(\alpha, x, l)} f\right)(y)=\alpha \cdot l(y) \cdot f(x+y) .
$$

Here we see clearly that we have the discrete analog of the usual irreducible representation of the Heisenberg Commutation relations: in integrated form, that representation is the action a) of multiplication by unitary characters, b) of translation operators, on $L^{2}$ of a real vector space.

Theorem 2. If $L$ is an ample invertible sheaf of separable type, then $\Gamma(X, L)$ is an irreducible $\mathscr{G}(L)$-module.

Proof. Let $K \subset \mathscr{G}(L)$ be a maximal level subgroup. Let $K$ be its image in $H(L)$, let $Y=X \mid K$, and let $M$ be the sheaf on $Y$ such that $L \cong \pi^{*} M$ ( $\pi: X \rightarrow Y$ being the projection). Then, as we have seen above, the maximality of $\tilde{K}$ implies that $M$ is an ample invertible sheaf of degree 1 . Therefore,

$$
\operatorname{dim} \Gamma(Y, M)=1 .
$$

On the other hand, by the theory of descent, $\pi^{*}$ maps the sections of $M$ onto the space of sections of $L$ invariant under $\tilde{K}$. Therefore

$$
\operatorname{dim} \Gamma(X, L)^{\tilde{K}}=1 . \quad \text { Q.E.D. }
$$

This irreducibility has an important application: it makes possible a simple direct construction of the variety of moduli of abelian varieties. In fact, this method of constructing the moduli space is nothing but the method of theta functions applied by BAILY in the classical case. It turns out that, when suitably algebraicized, it is perfectly applicable in all characteristics, at least to separably polarized abelian varieties, in char $\neq 2$. The basic idea is this: suppose we are given

(i) an abelian variety $X$,

(ii) a very ample invertible sheaf $L$ of separable type,

(iii) an isomorphism $\alpha$ of $\mathscr{G}(L)$ and $\mathscr{G}(\delta)$ which is the identity on the subgroups $k^{*}$.

Note that if i) and ii) are given, then there is only a finite set of data iii): so adding data of type (iii) means that one passes from the set of isomorphism classes of objects $(X, L)$ to a finite covering of this set.

Definition. Given $X$ and $L$ as in (i), (ii) above, data of type (iii) will be called $\vartheta$-structures on $(X, L)$.

If $X, L$ and a $\vartheta$-structure are given, they determine in a canonical way one projective embedding of $X[n . b$. not just an equivalence class of projectively equivalent embeddings]. 
Step $I$. Since $\Gamma(X, L)$ is the unique irreducible representation of $\mathscr{G}(L)$ and $V(\delta)$ is the unique irreducible representation of $\mathscr{G}(\delta)$, among representations which restrict to the natural character on $k^{*}$, there is an isomorphism

$$
\beta: \quad \Gamma(X, L) \stackrel{\sim}{\longrightarrow} V(\delta),
$$

unique up to scalar multiples, such that

all $x \in \mathscr{G}(L), s \in \Gamma(X, L)$.

$$
\beta\left\{U_{x}(s)\right\}=U_{\alpha(x)}(\beta(s))
$$

Step II. $\beta$ induces a completely unique isomorphism

$$
\boldsymbol{P}(\beta): \boldsymbol{P}[\Gamma(X, L)] \stackrel{\sim}{\longrightarrow}[V(\delta)]
$$

of projective spaces. Now pick, once and for all, a basis $\left\{X_{i}\right\}$ of $V(\delta)-$ say by ordering the elements $a_{1}, \ldots, a_{m}$ of $K(\delta)$ and taking $X_{i}$ as the delta function at $a_{i}$. Then this defines an isomorphism:

$$
\boldsymbol{P}(V(\delta)) \underset{\gamma}{\stackrel{\sim}{\longrightarrow}} \boldsymbol{P}_{m-1} .
$$

Here $m=$ cardinality $\{K(\delta)\}$

$$
=\text { degree }(L) \text {. }
$$

Step III. Since $L$ is very ample, there is a canonical embedding

$$
I: \quad X \hookrightarrow P[\Gamma(X, L)] .
$$

Then $J=\gamma \circ \boldsymbol{P}(\beta) \circ I$ is the canonical embedding of $X$ in $\boldsymbol{P}_{m}$ which we claimed existed.

The most striking consequence of having defined a canonical embedding is that this defines a canonical point in $\boldsymbol{P}_{\boldsymbol{m}-1}$ too: namely $J(e)$, where $e \in X$ is the identity point.

Definition. $J(e) \in \boldsymbol{P}_{m-1}$ will be called the theta-null point attached to $X, L$ and the given $\vartheta$-structure. The homogeneous coordinates of $J(e)$ will be called the theta-null values of $X, L$ and the given $\vartheta$-structure.

The ideas that we have indicated here will be fully developed in $\S 6$.

We can make the connection of our theory with the classical theory of theta-functions more explicit, and in particular motivate our terminology "theta-null values", in the following way. The classical theta functions (when $k=C$ ) arise as follows: if $g=\operatorname{dim} X$, then $C^{g}$ is the universal covering space of $X$. Let $\boldsymbol{o}_{\text {hol }}$ denote the trivial complex analytic invertible sheaf on $\boldsymbol{C}^{\mathrm{g}}$ : i.e., the sheaf of holomorphic functions itself. Let $\pi: \boldsymbol{C}^{\boldsymbol{g}} \rightarrow X$ be the projection. Then one chooses "very carefully" an isomorphism

$$
\lambda: \pi^{*}\left(L_{\mathrm{hol}}\right) \stackrel{\sim}{\longrightarrow} \boldsymbol{o}_{\mathrm{hol}}
$$


of sheaves on $\boldsymbol{C}^{\boldsymbol{g}}$ (here $L_{\text {hol }}$ denotes the complex analytic invertible sheaf corresponding to the algebraic invertible sheaf $L$ ). Then for all

$$
s \in \Gamma(X, L)=\Gamma\left(X, L_{\mathrm{hol}}\right)
$$

$\Theta_{s}=\lambda\left(\pi^{*}(s)\right)$ is a holomorphic function on $C^{g}$ : this is the theta function corresponding to $s$ and in this way $\Gamma(X, L)$ can be identified with a vector space of functions on $\boldsymbol{C}^{\mathrm{g}}$. We cannot imitate this procedure algebraically because $L \varsubsetneqq \boldsymbol{o}_{X}$. However, $L$ restricted to any finite set is isomorphic to $\boldsymbol{o}_{X}$ on that set. In particular we can use the structure of $\mathscr{G}(L)$ to construct nearly canonical isomorphisms of $L$ and $\boldsymbol{o}_{X}$ when restricted to $H(L)$.

Definition. Let $x$ be a closed point of $X$. Then let

$$
L(x)=L_{x} \otimes_{\boldsymbol{o}_{x}} \kappa(x),
$$

when $L_{x}$ is the stalk of $L$ at $x$ and $\kappa(x)$ is the residue field of $o_{x}$.

Now choose:

a) a $\vartheta$-structure

$$
\alpha: \mathscr{G}(L) \stackrel{\sim}{\longrightarrow} \mathscr{G}(\delta) \text { for } L,
$$

b) an isomorphism

$$
\lambda_{0}: L(0) \stackrel{\sim}{\longrightarrow} k .
$$

Let the $\vartheta$-structure induce the isomorphism $\bar{\alpha}$ :

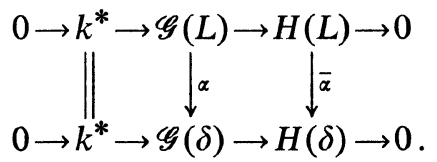

There is a canonical section of $\mathscr{G}(\delta)$ over $H(\delta)$ obtained by mapping $(x, l) \in H(\delta)$ back to $(1, x, l) \in \mathscr{G}(\delta)$. This induces a section $\sigma: H(L) \rightarrow \mathscr{G}(L)$. In particular, for all $w \in H(L)$, this gives us an element $\sigma(w)=\left(w, \varphi_{w}\right) \in \mathscr{G}(L)$, where $\varphi_{w}$ is an isomorphism:

$$
\varphi_{w}: L \rightarrow T_{w}^{*}(L) .
$$

Now we can use $\varphi_{w}$ to define the composite isomorphism:

$$
L(w)=T_{w}^{*} L(0) \underset{\varphi_{w}(0)}{\sim} L(0) \underset{\lambda_{0}}{\sim} \underset{\sim}{\sim} k .
$$

Call this $\lambda_{w}$. The collection of isomorphisms $\left\{\lambda_{w}\right\}$ is nothing but an isomorphism of the two sheaves $L$ and $\boldsymbol{o}_{X}$ restricted to $H(L)$.

Definition. For all $z \in H(\delta)$ and $s \in \Gamma(X, L)$, let $w=\bar{\alpha}^{-1}(z)$ and then define

$$
\Theta_{[s]}(z)=\lambda_{w}\left(\left.s\right|_{w}\right)=\lambda_{0}\left(\left.\varphi_{w}^{-1}\left(T_{w}^{*} s\right)\right|_{0}\right) .
$$


(Here $\left.s\right|_{w}$ denotes the image of the section $s$ in $L(w)$.) The square brackets around $s$ are inserted to indicate the connection with the classical notation for theta functions.

We now have a most surprising state of affairs. If a $\vartheta$-structure on $L$ is chosen, then we have 2 maps:

$$
\Gamma(X, L) \underset{\beta}{\stackrel{\sim}{\longrightarrow}}\{k \text {-valued functions on } K(\delta)\}=V(\delta),
$$

$$
\Gamma(X, L) \underset{\vartheta}{\longrightarrow}\{k \text {-valued functions on } H(\delta)\}
$$

both uniquely determined up to the ever-present scalar. For one thing, this means that we can combine the two, and obtain $\vartheta \circ \beta^{-1}$ : a transformation taking functions on $K(\delta)$ to functions on $H(\delta)$. We will work this out shortly. But first notice the essential contrast between the two maps: the first is a purely group-theoretic affair and should reflect well the group-theoretic properties of $X-$ as we will see in $\S 3$. The second, like the concept of theta functions, is just a natural way of converting sections of $L$ into functions by "evaluating" at points, so that it will respect multiplication of sections - i.e., it will extend to a homomorphism on the homogeneous coordinate ring $\oplus \Gamma\left(X, L^{n}\right)$ to the ring of functions on $H(\delta)$. The next step is to show that $\vartheta$ too has good grouptheoretic properties, even if it is not defined by such properties:

Theorem 3. Let $s \in \Gamma(X, L)$ and $y \in \mathscr{G}(L)$. Assume that

Then

$$
\alpha(y)=(\beta, x, l), \quad \beta \in k^{*}, \quad x \in K(\delta), \quad l \in \widehat{K(\delta)} .
$$

$$
\Theta_{\left[U_{y} s\right]}\left(x^{\prime}, l^{\prime}\right)=\beta \cdot\left(l^{\prime}-l\right)(x) \cdot \Theta_{[s]}\left(x^{\prime}-x, l^{\prime}-l\right) \text {. }
$$

Proof. The linear mapping

$$
s \mapsto \lambda_{0}\left(\left.s\right|_{0}\right)
$$

defines a linear functional $\bar{\lambda}: \Gamma(X, L) \rightarrow k$. Then, if $w^{\prime} \in H(L),\left(x^{\prime}, l^{\prime}\right)=$ $\bar{\alpha}\left(w^{\prime}\right)$, we have by definition:

$$
\begin{aligned}
\Theta_{[s]}\left(x^{\prime}, l^{\prime}\right) & =\lambda_{w^{\prime}}\left(\left.s\right|_{w^{\prime}}\right) \\
& =\lambda_{0}\left(\left.\varphi_{w^{\prime}}^{-1}\left(T_{w^{\prime}}^{*}(s)\right)\right|_{0}\right) \\
& =\tilde{\lambda}\left(U_{y^{\prime}}^{-1}(s)\right)
\end{aligned}
$$

if $y^{\prime}=\left(w^{\prime}, \varphi_{w^{\prime}}\right) \in \mathscr{G}(L)$. But $y^{\prime}$ is the unique element of $\mathscr{G}(L)$ over $w^{\prime}$ in the section described above. Now:

$$
\begin{aligned}
\Theta_{\left[U_{y}(s)\right]}\left(x^{\prime}, l^{\prime}\right) & =\tilde{\lambda}\left(U_{y^{\prime}}^{-1} U_{y}(s)\right) \\
& =\tilde{\lambda}\left(U_{y^{\prime-1} \cdot y}(s)\right) .
\end{aligned}
$$


But let $y^{-1} \cdot y^{\prime}=\gamma \cdot y^{\prime \prime}$, where $\gamma \in k^{*}$ and $y^{\prime \prime}$ is an element of $\mathscr{G}(L)$ in the distinguished section over $H(L)$. Then

$$
\begin{aligned}
\gamma \cdot \alpha\left(y^{\prime \prime}\right) & =\alpha(y)^{-1} \cdot \alpha\left(y^{\prime}\right) \\
& =(\beta, x, l)^{-1} \cdot\left(1, x^{\prime}, l^{\prime}\right) \\
& =\left(\beta^{-1} \cdot l(x) \cdot l^{\prime}(x)^{-1}, x^{\prime}-x, l^{\prime}-l\right) .
\end{aligned}
$$

Therefore, $\gamma^{-1}=\beta \cdot\left(l^{\prime}-l\right)(x)$, and $\alpha\left(y^{\prime \prime}\right)=\left(1, x^{\prime}-x, l^{\prime}-l\right)$. This implies that

$$
\begin{aligned}
\Theta_{\left[U_{y}(s)\right]}\left(x^{\prime}, l^{\prime}\right) & =\gamma^{-1} \cdot \tilde{\lambda}\left(U_{y^{\prime \prime}}^{-1}(s)\right) \\
& =\beta \cdot\left(l^{\prime}-l\right)(x) \cdot \Theta_{[s]}\left(x^{\prime}-x, l^{\prime}-l\right) . \quad \text { Q.E.D. }
\end{aligned}
$$

Definition. Let $\mathscr{G}(\delta)$ act on the vector space of functions on $H(\delta)$ by

$$
\left\{U_{(\beta, x, l)} f\right\}\left(x^{\prime}, l^{\prime}\right)=\beta \cdot\left(l^{\prime}-l\right)(x) \cdot f\left(x^{\prime}-x, l^{\prime}-l\right) .
$$

Corollary 1. With respect to the above action, we have

$$
\Theta_{\left[U_{y}(s)\right]}=U_{\alpha(y)}\left(\Theta_{[s]}\right) .
$$

Corollary 2. Either $\Theta_{[s]}=0$ for all $s \in \Gamma(X, L)$, or $\Theta_{[s]}=0$ only if $s=0$.

Proof. This follows because the kernel of $\Theta$ is a subspace of $\Gamma(X, L)$ invariant under $\mathscr{G}(L)$.

Corollary 3. Express the null-values of the functions $\Theta_{[s]}$ by the formula:

$$
\Theta_{[s]}(0,0)=\sum_{z \in K(\delta)}(\beta s)(z) \cdot q_{L}(z)
$$

with a suitable $k$-valued function $q_{L}$ on $K(\delta)$ i.e. $\left\{q_{L}(a)\right\}$ is the set of thetanull values of $X, L ; c f . p .298$. Then the transformation $\Theta \circ \beta^{-1}$ is given by:

$$
\left.\Theta_{[\beta-1} f\right](x, l)=\sum_{z \in K(\delta)} l(x-z) \cdot f(z-x) \cdot q_{L}(z) .
$$

Proof. Use the theorem with $x^{\prime}=l^{\prime}=0, \beta=1$, and the signs of $x, l$ reversed, to obtain

$$
\Theta_{\left[U_{y}(s)\right]}(0,0)=l(x)^{-1} \cdot \Theta_{[s]}(x, l) .
$$

Put $s=\beta^{-1} f$, use our expansion for the null-values and the Corollary comes out. Q.E.D.

Another consequence of the irreducibility of $\Gamma(X, L)$ under $\mathscr{G}(L)$ is that isogenies between abelian varieties induce canonical maps between corresponding linear systems. Suppose we are in the following situation, 
which we studied in the first part of this section:

$$
\begin{aligned}
& X \stackrel{\pi}{\longrightarrow} Y \\
& L \underset{\alpha}{\stackrel{\cdots}{\longrightarrow}} M .
\end{aligned}
$$

Here $\pi$ is a separable isogeny, $L$ and $M$ are ample invertible sheaves of separable type and $\alpha$ is an isomorphism $\alpha: \pi^{*} M \stackrel{\sim}{\sim} L$. Then $(\pi, \alpha)$ induce a linear map:

$$
\pi^{*}: \quad \Gamma(Y, M) \rightarrow \Gamma(X, L) .
$$

And, as we saw, $(\pi, \alpha)$ induces an isomorphism:

$$
\tau: \frac{\mathscr{G}(L)^{*}}{\tilde{K}} \stackrel{\sim}{\longrightarrow} \mathscr{G}(M),
$$

where

i) $\tilde{K}$ is the level subgroup over $\operatorname{ker}(\pi)$ which is the descent data on $L$ for $\pi$ associated to the descended sheaf $M$,

ii) $\mathscr{G}(L)^{*}$ is the group of elements of $\mathscr{G}(L)$ lying over the subgroup $\pi^{-1}[H(M)]$ in $X$,

iii) $\mathscr{G}(L)^{*}=$ centralizer of $\tilde{K}$.

Let $\delta_{M}$ and $\delta_{L}$ be types of $M$ and $L$ respectively and let

$$
\begin{array}{ll}
j_{L}: & \mathscr{G}(L) \stackrel{\sim}{\longrightarrow} \mathscr{G}\left(\delta_{L}\right) \\
j_{M}: & \mathscr{G}(M) \stackrel{\sim}{\longrightarrow} \mathscr{G}\left(\delta_{M}\right)
\end{array}
$$

be --structures. Let the $j$ 's induce isomorphisms:

$$
\begin{aligned}
\beta_{L}: \quad \Gamma(X, L) \stackrel{\sim}{\longrightarrow} V\left(\delta_{L}\right) \\
\beta_{M}: \quad \Gamma(Y, M) \stackrel{\sim}{\longrightarrow}\left(\delta_{M}\right) .
\end{aligned}
$$

The problem arises: what is the composite map $A=\beta_{L} \circ \pi^{*} \circ \beta_{M}^{-1}$ :

$$
V\left(\delta_{M}\right) \stackrel{\beta_{M}^{-1}}{\longrightarrow} \Gamma(Y, M) \stackrel{\pi^{*}}{\longrightarrow} \Gamma(X, L) \stackrel{\beta_{L}}{\longrightarrow} V\left(\delta_{L}\right) .
$$

Theorem 4. Assume $j_{L}(\tilde{K})$ is a subgroup of the form $\{1\} \times K_{1} \times K_{2}$, where $K_{1} \subset K\left(\delta_{L}\right), K_{2} \subset \widehat{K\left(\delta_{L}\right)}$. Let

$$
\begin{aligned}
& K_{1}^{\perp}=\left\{x \in K\left(\delta_{L}\right) \mid l(x)=1, \text { all } l \in K_{2}\right\} \\
& K_{2}^{\perp}=\left\{l \in K \widehat{\left(\delta_{L}\right)} \mid l(x)=1, \text { all } x \in K_{1}\right\} .
\end{aligned}
$$

Then $j_{L}\left(\mathscr{G}(L)^{*}\right)=k^{*} \times K_{1}^{\perp} \times K_{2}^{\perp}$, and $K_{1}^{\perp} / K_{1}$ and $K_{2}^{\perp} / K_{2}$ are canonically dual. Assume that there is an isomorphism

$$
\sigma: K_{1}^{\perp} / K_{1} \stackrel{\sim}{\longrightarrow}\left(\delta_{M}\right)
$$


such that if $\hat{\sigma}: K \frac{1}{2} / K_{2} \rightarrow \widehat{K\left(\delta_{M}\right)}$ is dual to $\sigma$, then the following diagram commutes:

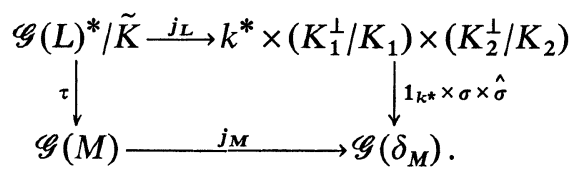

Then there is a scalar $\lambda$ such that for all $f \in V\left(\delta_{M}\right)$,

all $x \in K\left(\delta_{L}\right)$.

$$
A f(x)=\left\{\begin{array}{ccc}
0 & \text { if } & x \notin K_{1}^{\perp} \\
\lambda f(\sigma x) & \text { if } & x \in K_{1}^{\perp}
\end{array}\right.
$$

Proof. Notice that $\pi^{*}$ is injective and that its image is $\Gamma(X, L)^{\tilde{K}}$, the subspace of $\tilde{K}$-invariants. Moreover, $\Gamma(X, L)^{\tilde{\mathbf{K}}}$ is a module over

$$
\mathscr{G}^{\prime}=\frac{\mathscr{G}(L)^{*}}{\tilde{K}} .
$$

In fact, $\Gamma(X, L)^{\tilde{\mathbf{R}}}$ as $\mathscr{G}^{\prime}$-module is isomorphic to $\Gamma(Y, M)$ as $\mathscr{G}(M)$ module. Therefore, $\Gamma(X, L)^{\tilde{K}}$ is an irreducible $\mathscr{G}^{\prime}$-module. This means that $A$ is characterized, up to a scalar, by the 2 properties:

i) $\operatorname{Im}(A)=$ subspace of $V\left(\delta_{L}\right)$ of $\{1\} \times K_{1} \times K_{2}$-invariants.

ii) If $(\alpha, x, l) \in k^{*} \times K_{1}^{\perp} \times K_{2}^{\perp}$, then all $f \in V\left(\delta_{M}\right)$.

$$
U_{(\alpha, x, l)}(A f)=A\left(U_{(\alpha, \sigma x, \hat{\sigma} l)} f\right),
$$

It remains to check i) and ii) for the map $A$ defined by our formula. As for i), the formula gives

$$
\operatorname{Im}(A)=\left\{\begin{array}{l|l}
f \in V\left(\delta_{L}\right) & \begin{array}{l}
f \equiv 0 \text { outside } K_{1}^{\perp} \\
f \text { invariant under translations by elements of } K_{1}
\end{array}
\end{array}\right\}
$$

which is clearly the space of $\{1\} \times K_{1} \times K_{1}^{1}$-invariants. ii) is checked in a straightforward way too. Q.E.D.

\section{§2. Symmetric Invertible Sheaves}

As in $\S 1, l: X \rightarrow X$ will denote the inverse morphism on an abelian variety $X$. We assume in this section that char $(k) \neq 2$.

Definition. An invertible sheaf $L$ on an abelian variety $X$ is symmetric if $\imath^{*} L \cong L$.

Just as isomorphisms of $L$ with $T_{x}^{*} L$ were involved in the descent of $L$ with respect to isogenies $X \rightarrow Y$, so isomorphisms of $L$ and $\iota^{*} L$ are involved in the descent of $L$ with respect to $X \rightarrow X /\{1, l\}$.

21 Invent. math., Bd. 1 
Definition. If $X$ is an abelian variety, then the quotient of $X$ by the involution $l$ will be denoted by $K_{X}$, the Kummer variety of $X$. Moreover, $X^{f}$ will denote the open subset of $X$ consisting of $X$ minus its points of order 2 , and $K_{X}^{f}$ will be the quotient of $X^{f}$ by $l$.

Note that $X^{f}$ is just the set of points of $X$ where $\{1, \imath\}$ acts freely. If $\operatorname{dim} X \geqq 2$, then $K_{X}^{f}$ is just the set of non-singular points of $K_{X}$ and also the maximal open set over which

is flat.

$$
\pi: \quad X \rightarrow K_{X}
$$

If $L$ is a symmetric invertible sheaf, and

$$
\varphi: L \stackrel{\sim}{\longrightarrow} L
$$

is an isomorphism of $L$ with $\imath^{*} L$, then for all closed points $x \in X, \varphi$ restricts to an isomorphism:

$$
\varphi(x): \quad L(x) \stackrel{\sim}{\longrightarrow} \imath^{*} L(x)=L(-x) .
$$

We can always uniquely normalize $\varphi$ by demanding that $\varphi(0)$ is the identity map from $L(0)$ to $L(0)$. Look at the composition:

$$
L \stackrel{\varphi}{\longrightarrow} \imath^{*} L \stackrel{\imath^{*} \varphi}{\longrightarrow} l^{*}\left(\imath^{*} L\right)=L .
$$

In general, $\imath^{*} \varphi \circ \varphi$ must be given by multiplication by a constant $\alpha$ : but if $\varphi(0)$ is the identity, then $\imath^{*} \varphi \circ \varphi$ acts as the identity on $L(0)$, so $\alpha=1$.

Definition. $\varphi$ is a normalized isomorphism of $L$ and $\imath^{*} L$ if $\varphi(0)=$ identity.

Definition. Let $\varphi: L \rightarrow \imath^{*} L$ be the normalized isomorphism. Let $x \in X$ be a point of order 2. Define $e_{*}^{L}(x)$ to be the scalar $\alpha$ such that $\varphi(x)$ is multiplication by $\alpha(n . b$. since $2 x=0, L(-x)=L(x))$.

First Properties.

$$
\begin{array}{ll}
\text { i) } & e_{*}^{L}(x)= \pm 1, \quad e_{*}^{L}(0)=+1 . \\
\text { ii) } & e_{*}^{L \otimes M}(x)=e_{*}^{L}(x) \cdot e_{*}^{M}(x) .
\end{array}
$$

iii) If $\varphi: X \rightarrow Y$ is a homomorphism, and $L$ is a symmetric invertible sheaf on $Y$, then

for all $x \in X$ of order 2 .

$$
e_{*}^{\varphi^{*} L}(x)=e_{*}^{L}(\varphi(x))
$$

iv) If $\hat{X}$ is dual to $X$, let

$$
e_{2}: \quad X_{2} \times \hat{X}_{2} \rightarrow\{ \pm 1\}
$$


be the canonical pairing. If $x \in X_{2}$, and $\alpha \in \hat{X}_{2}$, and if $\alpha$ corresponds to an invertible sheaf $L$ of order 2 on $X$, then $L$ is symmetric and

$$
e_{*}^{L}(x)=e_{2}(x, \alpha) \text {. }
$$

Proofs. ii) and iii) are obvious. As for i), note that if $\varphi: L \stackrel{\sim}{\sim} \imath^{*} L$ is the normalized isomorphism, then $\imath^{*} \varphi \circ \varphi=$ identity, so for all closed points $x \in X, \varphi(-x) \circ \varphi(x)=$ identity. To prove iv), note that for any closed point $\alpha \in \hat{X}$ corresponding to a sheaf $L,-\alpha$ corresponds to $\imath^{*} L$. Hence $\alpha \in \hat{X}_{2}$ implies $L \cong \imath^{*} L$. Now recall the definition of $e_{2}$ (cf. [7], pp. $188-189)$ : let $\varphi$ be an isomorphism

$$
(2 \delta)^{*} L \longrightarrow o_{X} \text {. }
$$

Then if $\sigma$ is the canonical isomorphism, the following diagram commutes up to a factor $e_{2}(x, \alpha)$ :

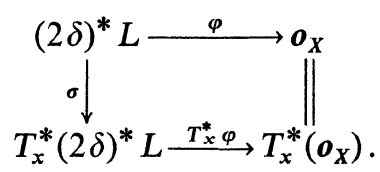

In particular, let $y \in X$ be such that $2 y=x$. Then looking at this diagram at the point $y$ we see that $\varphi(y)$ and $\varphi(-y)$ are maps from

$$
L(x)=L( \pm 2 y) \cong(2 \delta)^{*} L( \pm y) \rightarrow \boldsymbol{o}_{X}( \pm y)
$$

which differ from each other by $e_{2}(x, \alpha)$. On the other hand, if $\psi$ : $L \stackrel{\sim}{\sim} l^{*} L$ is the normalized isomorphism, then

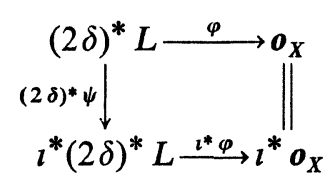

commutes (look at the diagram at 0 ). Therefore $\varphi(y)$ and $\varphi(-y)$ differ by $\left[(2 \delta)^{*} \psi\right](y)$, i.e., by $\psi(x)$. And $\psi(x)$ is by definition multiplication by $e_{*}^{L}(x)$. Q.E.D.

Proposition 1. Let $L$ be an invertible sheaf on $X$, and let $\pi: X \rightarrow K_{X}$ be the projection. Then $L$ is of the form $\pi^{*} M$ for some invertible sheaf $M$ on $K_{X}$ if and only if $L$ is symmetric and $e_{*}^{L}(x)=1$ for all points $x \in X$ of order 2.

Definition. A sheaf $L$ satisfying the conditions of this Proposition will be called totally symmetric.

$21^{*}$ 
Proof of Proposition. If $L \cong \pi^{*} M$, then it follows immediately that $L$ is symmetric and $e_{*}^{L}(x)=1$, all $x$. Conversely, assume these conditions on $L$ and let $D$ be a divisor on $X$ such that $L \cong \boldsymbol{o}_{X}(D)$. Since $L$ is symmetric, $l^{-1}(D)$ is linearly equivalent to $D$ : say

Then:

$$
\imath^{-1}(D)=D+(f) \text {. }
$$

hence

$$
\begin{aligned}
D & =l^{-1}\left(l^{-1}(D)\right) \\
& =l^{-1} D+l^{-1}(f) \\
& =l^{-1} D+\left(l^{*} f\right),
\end{aligned}
$$

$$
\left(l^{*} f\right) \cdot f=\alpha, \quad \alpha \in k^{*} \text {. }
$$

Replacing $f$ by $\sqrt{\alpha} \cdot f$, we may assume $\imath^{*} f=f^{-1}$. Applying HiLberT's theorem 90 to the action of the group $\left(1, \imath^{*}\right)$ on the function field $k(X)$ of $X$, it follows that

for some $g \in k(X)$. Now let

$$
f=l^{*} g \cdot g^{-1}
$$

$$
D^{\prime}=D-(g) \text {. }
$$

Then it follows immediately that $l^{-1}\left(D^{\prime}\right)=D^{\prime}$. Now if we restrict $D^{\prime}$ to the subset $X^{f}$ where $l$ acts freely, this implies that $D^{\prime}=\pi^{-1}(E)$ for some divisor $E$ on $K_{X}^{f}$. But this is not automatic at points $x$ of order 2. However, $L \cong \boldsymbol{o}_{X}\left(D^{\prime}\right)$ and we have assumed the existence of an isomorphism

$$
\varphi: L \stackrel{\sim}{\longrightarrow} \imath^{*} L
$$

such that $\varphi(x)$ is the identity for all $x$ of order 2 . In other words, if $s_{x} \in L_{x}$ is a generator of the stalk of $L$ at $x$, then $\varphi\left(s_{x}\right)$ differs from $l^{*}\left(s_{x}\right)$ by an element of $\boldsymbol{m}_{\boldsymbol{x}} \cdot L_{\boldsymbol{x}}\left(\boldsymbol{m}_{\boldsymbol{x}}=\right.$ the maximal ideal of the local ring at $\left.x\right)$. Replacing $L$ by $\boldsymbol{o}_{X}\left(D^{\prime}\right)$, this means that there is an $\alpha \in k^{*}$ such that if $f_{x}$ is a local equation for $D^{\prime}$ at a point $x$ of order 2 , then

$$
\imath^{*} f_{x} \equiv \alpha f_{x} \quad\left(\bmod f_{x} \cdot \boldsymbol{m}_{x}\right) \text {. }
$$

It is clear that $\alpha=+1$ or -1 . If $\alpha=-1$, we replace $D^{\prime}$ by $D^{\prime}+(h)$, where $h$ is an element of $k(X)$ such that $l^{*} h=-h$, so that we may assume $\alpha=+1$. Now consider:

$$
f_{x}^{\prime}=\frac{\imath^{*} f_{x} \cdot f_{x}}{l^{*} f_{x}+f_{x}} .
$$

$f_{x}^{\prime}$ is still a local equation for $D^{\prime}$, and $\imath^{*} f_{x}^{\prime}=f_{x}^{\prime}$. Therefore, $f_{x}^{\prime}$ is a local equation at $\pi(x)$ on $K_{X}$ for a divisor $E$ such that $\pi^{-1} E=D^{\prime}$ at $x$. In this way, we see that there is a globally defined (CARTIER) divisor $E$ on $K_{X}$ such that $\pi^{-1} E=D^{\prime}$, and hence $\pi^{*}\left(\boldsymbol{o}_{\mathbf{K}_{X}}(E)\right) \cong \boldsymbol{o}_{X}\left(D^{\prime}\right) \cong L$. Q.E.D. 
Note that if $L$ is any invertible sheaf, then $L \otimes \imath^{*} L$ is totally symmetric: so that there are plenty of very ample totally symmetric sheaves on $X$. A useful remark about totally symmetric sheaves is that if $L_{1}, L_{2}$ are algebraically equivalent totally symmetric sheaves, then $L_{1} \cong L_{2}$. Put in another way, no non-trivial totally symmetric sheaves are algebraically equivalent to zero. In fact, let $L_{\alpha}$ be the sheaf corresponding to the closed point $\alpha \in \hat{X}$. Then $l^{*} L_{\alpha} \cong L_{-\alpha}$, so $L$ is symmetric if and only if $2 \alpha=e$. Then my assertion follows from property (iv) above. Therefore, totally symmetric invertible sheaves are a very convenient class of sheaves to work with. In most of the sequel, we shall stick with this type of sheaf and the corresponding projective embeddings.

Following further the ideas in the proof of Proposition 1, we get:

Proposition 2. Let $D$ be a symmetric divisor, i.e., $l^{-1}(D)=D$, and let $L=o_{X}(D)$. Then for all points $x$ of order 2:

$$
e_{*}^{L}(x)=(-1)^{m(x)-m(0)}
$$

where $m(y)=$ multiplicity of $D$ at $y$, for all closed points $y \in X$.

Proof. Since $D$ is the difference of 2 symmetric effective divisors, it suffices to prove the Proposition when $D$ is itself effective. Now define $\varphi$ to be the composition:

$$
L=o_{X}(D)=o_{X}\left(l^{-1}(D)\right) \cong l^{*}\left[o_{X}(D)\right]=l^{*} L .
$$

I claim that for all points $y$ of order 2 (including 0$), \varphi(y)$ is multiplication by $(-1)^{m(y)}$ : hence $(-1)^{m(0)} . \varphi$ is the normalized isomorphism of $\imath^{*} L$ and $L$ and the result follows. To see this, let $f_{y}$ be a local equation of $D$ at $y$. Then $\imath^{*} f_{y}=\alpha \cdot f_{y}$ where $\alpha$ is a unit in $\boldsymbol{o}_{y, x}$. Since $\imath^{*} \alpha=\alpha^{-1}$, and $\imath^{*}$ operates trivially on $\boldsymbol{o}_{y} / \boldsymbol{m}_{y}$, it follows that $\alpha \equiv \pm 1\left(\bmod \boldsymbol{m}_{y}\right)$. On the other hand, by definition, $\varphi$, at $y$, maps the generator $f_{y}$ of the stalk $L_{y}$ to the generator $\imath^{*} f_{y}$ of the stalk $\left(\imath^{*} L\right)_{y}$. Therefore $\varphi(y)$ is multiplication by the scalar $\alpha \bmod \boldsymbol{m}_{\boldsymbol{y}}$. Finally, to compute $\alpha$ directly, let $m=m(y)$, so that

$$
f_{y} \in \boldsymbol{m}_{y}^{m}-\boldsymbol{m}_{y}^{m+1} \text {. }
$$

The automorphism $l$ acts on $\boldsymbol{m}_{\boldsymbol{y}} / \boldsymbol{m}_{y}^{2}$ as multiplication by -1 , hence it acts on $\boldsymbol{m}_{y}^{m} / \boldsymbol{m}_{y}^{m+1}$ as multiplication by $(-1)^{m}$. Then $\alpha \bmod \boldsymbol{m}_{y}$ is determined by the congruence:

$$
\alpha \cdot f_{y}=\imath^{*} f_{y} \equiv(-1)^{m} f_{y}\left(\bmod m_{y}^{m+1}\right) . \quad \text { Q.E.D. }
$$

The analysis of symmetry in this section has proceeded along quite different lines from the study of isomorphisms of $L$ with its translates given in the first section. Our next goal is to reinterpret the symmetry 
condition in general and $e_{*}^{L}$ in particular in group-theoretic terms involving $\mathscr{G}(L)$ (actually, involving the groups $\mathscr{G}\left(L^{n}\right)$ also). Now assume that $L$ is an ample symmetric sheaf of separable type.

Definition. Let $\psi: L \stackrel{\sim}{\sim} t^{*} L$ be any isomorphism. Then if $(x, \varphi) \in \mathscr{G}(L)$, consider the composition:

Set

$$
\begin{aligned}
L \stackrel{\sim}{\sim} \imath^{*} L \stackrel{\imath^{*}(\varphi)}{\longrightarrow} \imath^{*} T_{x}^{*} L & \\
& T_{-x}^{*} \imath^{*} L \stackrel{T^{*}-x \psi}{\longleftarrow} T_{-x}^{*} L .
\end{aligned}
$$

$$
\delta_{-1}((x, \varphi))=\left(-x,\left(T_{-x}^{*} \psi\right)^{-1} \circ\left(l^{*} \varphi\right) \circ \psi\right) .
$$

Note that $\delta_{-1}$ is independent of the choice of $\psi$. One checks immediately that $\delta_{-1}$ is a homomorphism from $\mathscr{G}(L)$ to $\mathscr{G}(L)$ and that $\delta_{-1} \circ \delta_{-1}$ is the identity. In fact, $\delta_{-1}$ is an automorphism of $\mathscr{G}(L)$ that fits into the diagram:

$(*)_{-1}$

$$
\begin{aligned}
& 0 \rightarrow k^{*} \rightarrow \mathscr{G}(L) \rightarrow H(L) \rightarrow 0 \\
& \downarrow \text { id } \downarrow \delta-1 \quad \downarrow-1 \\
& 0 \rightarrow k^{*} \rightarrow \mathscr{G}(L) \rightarrow H(L) \rightarrow 0 \text {. }
\end{aligned}
$$

$\delta_{-1}$ is, then, the reflection of the inverse $t$ in the group $\mathscr{G}(L)$. The notation $\delta_{-1}$ is motivated by the following:

Definition. If $z \in \mathscr{G}(L)$, and $n$ is any integer, let

$$
\delta_{n}(z)=(z)^{\frac{n^{2}+n}{2}} \cdot\left[\delta_{-1}(z)\right]^{\frac{n^{2}-n}{2}} .
$$

It is a straightforward calculation to check that $\delta_{n}$ is a homomorphism, that $\delta_{n m}=\delta_{n} \circ \delta_{m}$ and that $\delta_{n}$ fits into a diagram:

$(*)_{n}$

$$
\begin{gathered}
0 \rightarrow k^{*} \rightarrow \mathscr{G}(L) \rightarrow H(L) \rightarrow 0 \\
n^{2} \text {-power } \\
0 \rightarrow k^{*} \rightarrow \mathscr{G}(L) \rightarrow H(L) \rightarrow 0 .
\end{gathered}
$$

We omit these calculations.

An important consequence of the existence of $\delta_{-1}$ is that we nearly have a canonical section of $\mathscr{G}(L)$ over $H(L)$. In fact, if $x \in H(L)$, then there will be exactly 2 elements $z \in \mathscr{G}(L)$ over $x$ such that

$$
\delta_{-1} z=z^{-1} \text {. }
$$

Starting with any $z_{0} \in \mathscr{G}(L)$ over $x$, it follows that $\delta_{-1} z_{0}=\alpha \cdot z_{0}^{-1}$, where $\alpha \in k^{*}$. The most general element over $x$ is of the form $\beta \cdot z_{0}, \beta \in k^{*}$; 
and

$$
\delta_{-1}\left(\beta z_{0}\right)=\beta^{2} \cdot \alpha \cdot\left(\beta z_{0}\right)^{-1}
$$

Hence if $\beta=\alpha^{-\frac{1}{2}}, \pm \beta \cdot z_{0}$ are the elements over $x$ satisfying formula (1).

Definition. Let $\mathscr{S}(L)=\left\{z \in \mathscr{G}(L) \mid \delta_{-1} z=z^{-1}\right\}$. If $(x, \varphi) \in \mathscr{S}(L)$, then $\varphi$ will be called a symmetric isomorphism of $L$ and $T_{x}^{*} L \delta_{-1}$ and $e_{*}$ are related by:

Proposition 3. Let $L$ be an ample symmetric invertible sheaf of separable type. Let $z \in \mathscr{G}(L)$ lie over a point $x \in H(L)$ of order 2. Then

$$
\delta_{-1}(z)=e_{*}^{L}(x) \cdot z
$$

Proof. Let $z=(x, \varphi)$, where $\varphi: L \stackrel{\sim}{\longrightarrow} T_{x}^{*} L$. Let $\psi: L \stackrel{\sim}{\longrightarrow} \imath^{*} L$ be a normalized isomorphism. Then $\delta_{-1} z=\left(x,\left(T_{x}^{*} \psi\right)^{-1} \circ\left(l^{*} \varphi\right) \circ \psi\right)$. To compare $\varphi$ and $\left(T_{x}^{*} \psi\right)^{-1} \circ\left(l^{*} \varphi\right) \circ \psi$, look at the induced maps that both define from $L(0)$ to $L(x)$. In fact, the latter map gives:

$$
L(0) \stackrel{\psi(0)}{\longrightarrow} L(0) \stackrel{\varphi(0)}{\longrightarrow} L(x) \stackrel{\psi(x)^{-1}}{\longrightarrow} L(x),
$$

which differs from $\varphi(0)$ by $e_{*}^{L}(x)=\psi(x)^{-1}$. Therefore, $\left(T_{x}^{*} \psi\right)^{-1} \circ\left(\imath^{*} \varphi\right) \circ \psi$ is the product of $\varphi$ by the scalar $e_{*}^{L}(x)$. Q.E.D.

Unfortunately, this Proposition does not allow us to recapture the invariant $e_{*}^{L}$ from $\delta_{-1}$ unless $H(L)$ contains all points of order 2 . In fact, there is yet another canonical homomorphism whose existence depends on the symmetry of $L$. This involves the relation between $\mathscr{G}(L)$ and $\mathscr{G}\left(L^{n}\right)$. First, however, we define a trivial homomorphism which does not require the symmetry of $L$ :

Definition. Let $L$ be an ample invertible sheaf of separable type, and let $n$ be an integer, $n \geqq 2$ such that char $(k) \chi n$. Let $(x, \varphi) \in \mathscr{G}(L)$.

Define

$$
\varepsilon_{n}(x, \varphi)=\left(x, \varphi^{\otimes n}\right)
$$

where $\varphi^{\otimes n}$ stands for the isomorphism

induced by $\varphi$.

$$
L^{n} \stackrel{\varphi^{\otimes n}}{\longrightarrow} T_{x}^{*} L^{n}
$$

This defines a homomorphism $\varepsilon_{n}$ fitting into the diagram:

$(* *)_{n}$

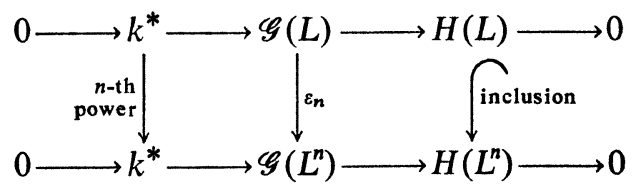

Also note that $H(L)$ and $H\left(L^{n}\right)$ are related by: 
Proposition 4. If $L$ is an arbitrary invertible sheaf on an abelian variety $X$, then

$$
\begin{array}{ll}
\text { and } & H\left(L^{n}\right)=\{x \mid n x \in H(L)\} \\
& H(L)=n \cdot H\left(L^{n}\right) .
\end{array}
$$

Proof. In fact, $H(L)$ and $H\left(L^{n}\right)$ are the kernels of the homomorphisms:

$$
\begin{array}{ll}
\Lambda(L): & X \rightarrow \hat{X} \\
\Lambda\left(L^{n}\right): & X \rightarrow \hat{X}
\end{array}
$$

mentioned at the beginning of $\S 1$. But

$$
\Lambda\left(L^{n}\right)=n \cdot \Lambda(L)
$$

(cf. [9], Ch. 6, p. 120). Therefore ker $\left[\Lambda\left(L^{n}\right)\right]$ is the set of closed points $x \in X$ such that $n \cdot x \in \operatorname{ker}[\Lambda(L)]$ : this gives the first statement. Then $H(L)$ equals $n \cdot H\left(L^{n}\right)$ since the group of closed points of $X$ is divisible. Q.E.D.

In contrast to $\varepsilon_{n}$, the symmetry of $L$ is involved in the existence of a non-trivial canonical homomorphism $\eta_{n}: \mathscr{G}\left(L^{n}\right) \rightarrow \mathscr{G}(L)$ fitting into the diagram:

$(* * *)_{n}$

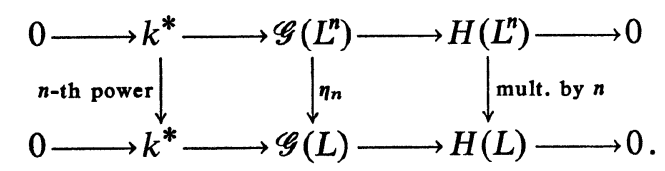

Definition (of $\eta_{n}$ ). Assume that $L$ is symmetric. Start with $z=$ $(x, \varphi) \in \mathscr{G}\left(L^{n}\right)$. Since $L$ is symmetric, there is an isomorphism

$$
\psi: L^{n^{2}} \stackrel{\sim}{\longrightarrow}(n \delta)^{*} L
$$

(cf. $§ 1$, (IV)). Consider the diagram:

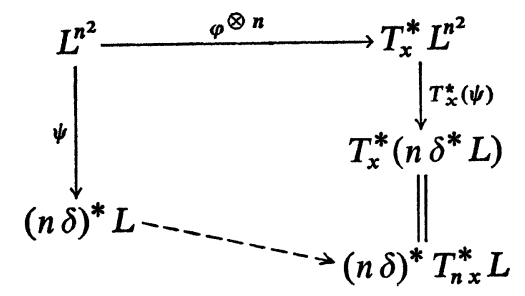

By the previous Proposition, $n x \in H(L)$, hence there is some isomorphism between $L$ and $T_{n x}^{*} L$. Then there is a unique isomorphism $\rho: L \stackrel{\sim}{\longrightarrow} T_{n x}^{*} L$ such that

$$
(n \delta)^{*} \rho=T_{x}^{*} \psi \circ \varphi^{\otimes n} \circ \psi^{-1}
$$


i.e., such that with $(n \delta)^{*} \rho$ along the dotted line, the above diagram commutes. Set

$$
\eta_{n}(x, \varphi)=(n x, \rho) .
$$

One checks easily that $\eta_{n}$ is independent of $\psi$, and is a homomorphism.

Proposition 5. Let $\delta_{m}^{\prime}: \mathscr{G}(L) \rightarrow \mathscr{G}(L)$ and $\delta_{m}^{\prime \prime}: \mathscr{G}\left(L^{n}\right) \rightarrow \mathscr{G}\left(L^{n}\right)$ be the homomorphisms defined above. Then

$$
\delta_{-1}^{\prime} \circ \eta_{n}=\eta_{n} \circ \delta_{-1}^{\prime \prime},
$$

$$
\delta_{-1}^{\prime \prime} \circ \varepsilon_{n}=\varepsilon_{n} \circ \delta_{-1}^{\prime}
$$

(hence $\delta_{m}$, for all $m$, commutes with $\eta$ and $\varepsilon$ ). Moreover,

$$
\begin{aligned}
\delta_{n}^{\prime} & =\eta_{n} \circ \varepsilon_{n}, \\
\delta_{n}^{\prime \prime} & =\varepsilon_{n} \circ \eta_{n} .
\end{aligned}
$$

Proof. (i) and (ii) are verified easily by writing out the definitions: this will be omitted. Also, (iii) follows from (iv). Namely, if we assume (iv), then:

$$
\begin{aligned}
\delta_{n}^{\prime} \circ \eta_{n} & =\eta_{n} \circ \delta_{n}^{\prime \prime} \\
& =\eta_{n} \circ \varepsilon_{n} \circ \eta_{n} .
\end{aligned}
$$

Since $\eta_{n}$ is surjective, (iii) must hold. Now (iv) is harder: note first that both $\delta_{n}^{\prime \prime}$ and $\varepsilon_{n} \circ \eta_{n}$ fit in as dotted lines in the diagram:

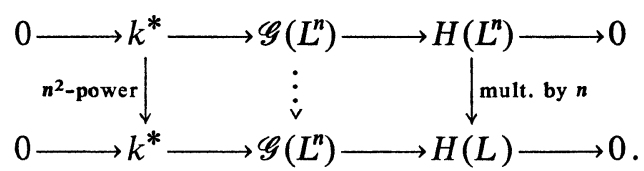

Therefore, there is a homomorphism $h: H\left(L^{n}\right) \rightarrow k^{*}$ such that

$$
\varepsilon_{n}\left(\eta_{n}(z)\right)=h(\bar{z}) \cdot \delta_{n}^{\prime \prime}(z)
$$

(here $z \in \mathscr{G}\left(L^{n}\right)$ and $\bar{z}$ is the image of $z$ in $H\left(L^{n}\right)$ ). But then:

$$
\begin{aligned}
h(\bar{z}) \cdot \delta_{-1}^{\prime \prime}\left(\delta_{n}^{\prime \prime} z\right) & =\delta_{-1}^{\prime \prime}\left[h(\bar{z}) \cdot \delta_{n}^{\prime \prime}(z)\right] \\
& =\delta_{-1}^{\prime \prime}\left[\varepsilon_{n}\left(\eta_{n} z\right)\right] \\
& =\varepsilon_{n}\left(\eta_{n}\left(\delta_{-1}^{\prime \prime} z\right)\right) \\
& =h\left(\overline{\delta_{-1}^{\prime \prime} z}\right) \cdot \delta_{n}^{\prime \prime}\left(\delta_{-1}^{\prime \prime} z\right) \\
& =h(-\bar{z}) \cdot \delta_{-n}^{\prime \prime} z \\
& =h(\bar{z})^{-1} \cdot \delta_{-1}^{\prime \prime}\left(\delta_{n}^{\prime \prime} z\right),
\end{aligned}
$$

hence $h(\bar{z})= \pm 1$ for all $z \in \mathscr{G}\left(L^{n}\right)$. In particular, $\varepsilon_{n}\left(\eta_{n}(z)\right)=\delta_{n}^{\prime \prime}(z)$ for all $z \in \mathscr{G}\left(L^{n}\right)$ such that $\bar{z} \in 2 H\left(L^{n}\right)$. 
Now suppose that we have a separable isogeny:

$$
f: \quad Y \rightarrow X
$$

and that we set $M=f^{*} L$. If we set $\mathscr{G}(M)^{*}$ equal to the subgroup of elements $z$ whose image in $H(M)$ is in $f^{-1}[H(L)]$, then as we know from $\S 1$,

$$
\mathscr{G}(L) \cong \mathscr{G}(M)^{*} / \tilde{K}
$$

for some level subgroup $\tilde{K}$. Similarly, $\mathscr{G}\left(L^{n}\right) \cong \mathscr{G}\left(M^{n}\right)^{*} / \tilde{K}_{n}$. It is easy to check that $\delta_{-1}^{\prime \prime}\left(\mathscr{G}\left(M^{n}\right)^{*}\right) \subset \mathscr{G}\left(M^{n}\right)^{*}, \varepsilon_{n}\left(\mathscr{G}(M)^{*}\right) \subset \mathscr{G}\left(M^{n}\right)^{*}, \eta_{n}\left(\mathscr{G}\left(M^{n}\right)^{*}\right) \subset$ $\mathscr{G}(M)^{*}$, and that the diagram $(s)$ :

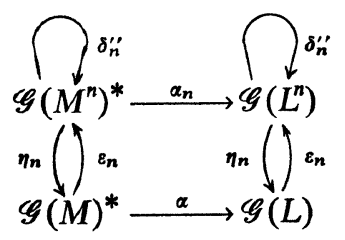

commute, (here $\alpha, \alpha_{n}$ stand for the canonical maps). Now suppose, for $z \in \mathscr{G}\left(M^{n}\right), \varepsilon_{n}\left(\eta_{n}(z)\right)$ differs from $\delta_{n}^{\prime \prime}(z)$ by $\tilde{h}(\bar{z})$. Then if $z \in \mathscr{G}\left(M^{n}\right)^{*}$,

$$
\begin{aligned}
\tilde{h}(\bar{z}) & =\alpha_{n}(\tilde{h}(\bar{z})) \\
& =\alpha_{n}\left(\varepsilon_{n}\left(\eta_{n}(z)\right) \cdot \delta_{n}^{\prime \prime}(z)^{-1}\right) \\
& =\varepsilon_{n}\left(\eta_{n}\left(\alpha_{n} z\right)\right) \cdot \delta_{n}^{\prime \prime}\left(\alpha_{n} z\right)^{-1} \\
& =h\left(\overline{\alpha_{n} z}\right) .
\end{aligned}
$$

Therefore, if every element of $f^{-1}\left(H\left(L^{n}\right)\right)$ is divisible by two in $H\left(M^{n}\right)$, it follows that $\tilde{h} \equiv 1$ on $f^{-1}\left(H\left(L^{n}\right)\right)$, hence $h \equiv 1$ on $H\left(L^{n}\right)$. It is easy to make this the case, however: choose $X=Y$ and $f=2 \delta$ for example. Then $M \cong L^{4}$, hence

$$
\begin{aligned}
H\left(M^{n}\right) & =\left\{x \mid 4 x \in H\left(L^{n}\right)\right\} \\
f^{-1}\left[H\left(L^{n}\right)\right] & =\left\{x \mid 2 x \in H\left(L^{n}\right)\right\} . \quad \text { Q.E.D. }
\end{aligned}
$$

Consider, in particular, $\eta_{2}$. This map alone seems to contain all the useful canonical data to be extracted from the symmetry of $L$. On the one hand, forming $\varepsilon_{2} \circ \eta_{2}$, we obtain $\delta_{2}: \mathscr{G}(L) \rightarrow \mathscr{G}(L)$. And since $\delta_{2}(z)=$ $z^{3} \cdot \delta_{-1}(z)$, we can also reconstruct $\delta_{-1}$ and hence all the maps $\delta_{n}$. On the other hand, unlike $\delta_{-1}$, we can always reconstruct $e_{*}^{L}$ from $\eta_{2}$ :

Proposition 6. Let $z \in \mathscr{G}\left(L^{2}\right)$ be an element of order 2 and let $x$ be its image in $H\left(L^{2}\right)$. Then $\eta_{2}(z)$ is in $k^{*}$ and

$$
\eta_{2}(z)=e_{*}^{L}(x)
$$


Proof. Let $z=(x, \varphi)$, where $\varphi$ is an isomorphism

Moreover, let

$$
\varphi: \quad L^{2} \stackrel{\sim}{\longrightarrow} T_{x}^{*} L^{2} .
$$

$$
\rho: \quad L \rightarrow \imath^{*} L
$$

be a normalized isomorphism, and let

$$
\psi: \quad L^{4} \rightarrow(2 \delta)^{*} L
$$

be an arbitrary isomorphism. Choose a point $y \in X$ such that $2 y=x$.

The first step is to consider the 2 maps:

$$
\varphi(y), \rho^{2}(y): L^{2}(y) \stackrel{\sim}{\longrightarrow} L^{2}(-y) .
$$

I claim that $\varphi(y)= \pm \rho^{2}(y)$. To see this, look at the diagram:

( $\beta)$

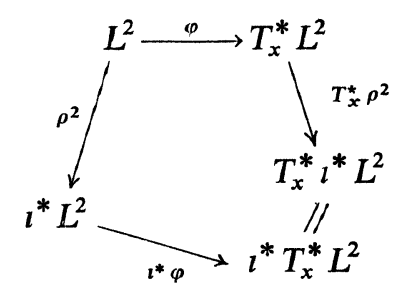

This diagram commutes: look at the induced maps at the origin

$(\beta)_{0}$

$$
\begin{gathered}
\left.L(0)^{2}\right|^{2} \stackrel{\varphi(x)}{\longrightarrow} L(x)^{2} \\
L(0)^{2} \stackrel{\varphi(x)}{\longrightarrow} L(x)^{2} .
\end{gathered}
$$

Since $\rho(0)=1$, and $\rho(x)=e_{*}^{L}(x)= \pm 1,(\beta)_{0}$ commutes, so $(\beta)$ commutes also. On the other hand, look at diagram $(\beta)$ at the point $y$. We find that

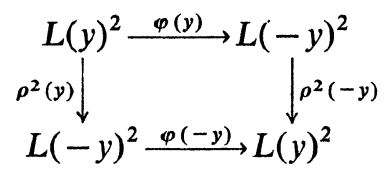

commutes. Now since $z=(x, \varphi)$ has order 2 , it follows that $T_{x}^{*} \varphi=\varphi^{-1}$. In particular, $\varphi(-y)=\varphi(y)^{-1}$. Moreover, since $\rho$ is normalized, we know that $l^{*} \rho=\rho^{-1}$. In particular, $\rho(-y)=\rho(y)^{-1}$. Putting all this together, we conclude:

Therefore, $\rho^{4}(y)=\varphi^{2}(y)$.

$$
\rho^{2}(y)= \pm \varphi(y) \text {. }
$$


What is the definition of $\eta_{2}(z)$ ? According to our recipe, in this case, $\eta_{2}(z)$ is the scalar $\alpha$ such that the diagram:

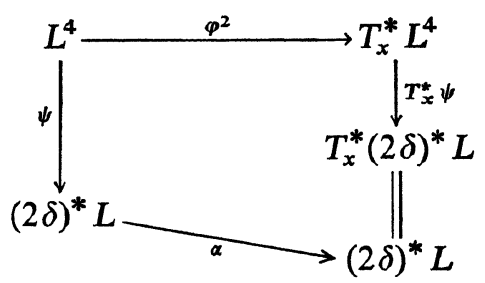

commutes. Therefore, $\eta_{2}(z)$ is the composite map:

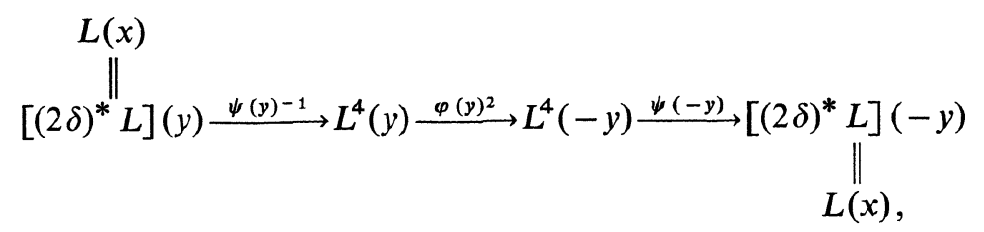

(i.e., identifying the scalar $\eta_{2}(z)$ with multiplication by this scalar). By our first result, it follows that

$$
\eta_{2}(z)=\psi(-y) \circ \rho(y)^{4} \circ \psi(y)^{-1} .
$$

Thirdly, consider the diagram:

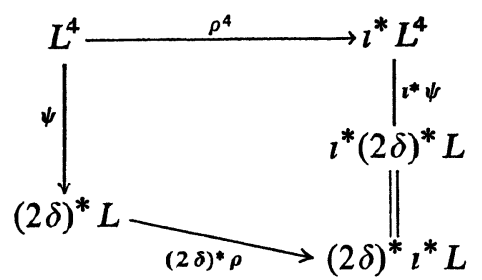

This diagram commutes: look at the induced maps at the origin

$$
\begin{gathered}
\psi(0) \downarrow \\
L(0) \stackrel{\rho(0)}{\longrightarrow} L(0) .
\end{gathered}
$$

Since $\rho(0) \doteq 1$, this induced diagram commutes, hence so does the full diagram of sheaves on $X$. Now look at the full diagram at the point $y$. We find that

$$
\rho(x)=\left[(2 \delta)^{*} \rho\right](y)=\psi(-y) \circ \rho(y)^{4} \circ \psi(y)^{-1} .
$$

But, by definition, $\rho(x)=e_{*}^{L}(x)$; while we just proved that $\psi(-y) \circ$ $\rho(y)^{4} \circ \psi(y)^{-1}=\eta_{2}(z)$. Q.E.D.

Corollary 1. Let $L$ be a symmetric invertible sheaf on an abelian variety $X$, and let $X_{2}$ denote the group of points of order 2 on $X$. Then $e_{*}^{L}$ 
is a quadratic function from $X_{2}$ to $\{ \pm 1\}$ whose associated bilinear form is $e^{\left(L^{2}\right)}$ (this is defined on $X_{2} \times X_{2}$ since $X_{2} \subset H\left(L^{2}\right)$ ). In other words:

$$
e_{*}^{L}(x+y)=e_{*}^{L}(x) \cdot e_{*}^{L}(y) \cdot e^{\left(L^{2}\right)}(x, y)
$$

Proof. Let $z, w$ be elements of $\mathscr{G}\left(L^{2}\right)$ over $x, y$ respectively such that $z^{2}=w^{2}=1$. Then

$$
\begin{aligned}
(z \cdot w)^{2} & =z \cdot w \cdot z \cdot w \\
& =z \cdot w \cdot z^{-1} \cdot w^{-1} \\
& =e^{\left(L^{2}\right)}(x, y) .
\end{aligned}
$$

Let $\beta$ be a square root of $e^{\left(L^{2}\right)}(x, y)$ in $k^{*}$. Then $\beta^{-1} \cdot z \cdot w$ is an element of order 2 in $\mathscr{G}\left(L^{2}\right)$ over $x+y$. Therefore

$$
\begin{aligned}
e_{*}^{L}(x+y) & \cdot e_{*}^{L}(x)^{-1} \cdot e_{*}^{L}(y)^{-1} \\
= & \eta_{2}\left(\beta^{-1} \cdot z \cdot w\right) \cdot \eta_{2}(z)^{-1} \cdot \eta_{2}(w)^{-1} \\
= & \eta_{2}(\beta)^{-1} \\
= & \beta^{-2} \\
= & e^{\left(L^{2}\right)}(x, y) . \quad \text { Q.E.D. }
\end{aligned}
$$

Note that all these members (except $\beta$ ) are \pm 1 . This is the reason why the pairing $e^{\left(L^{2}\right)}$, which is always skew-symmetric, is also symmetric on $X_{2} \times X_{2}$ and hence possible as the associated bilinear form to a quadratic form.

Corollary 2. Let $L$ be a symmetric invertible sheaf on an abelian variety $X$. Then $L$ is totally symmetric if and only if

$$
\operatorname{ker}\left(\eta_{2}\right)=\left\{z \in \mathscr{G}\left(L^{2}\right) \mid z^{2}=1\right\} .
$$

Proof. Immediate.

Corollary 3. Let $D$ be a symmetric divisor on an abelian variety $X$ of dimension $r$. Let $\Sigma_{+}$be the set of points of order 2 at which $D$ has even multiplicity, and let $\Sigma_{-}$be the set where $D$ has odd multiplicity. Then either

case i) \# $\left(\Sigma_{+}\right)=2^{2 r-s-1}\left(2^{s}+1\right)$, $\#\left(\Sigma_{-}\right)=2^{2 r-s-1}\left(2^{s}-1\right)$ for some integer $s, 0 \leqq s \leqq r$.

case ii) Same as case i) with $\Sigma_{+}, \Sigma_{-}$reversed.

case iii) \# $\left(\Sigma_{+}\right)=\#\left(\Sigma_{-}\right)=2^{2 r-1}$.

Proof. This follows from Prop. 2 of this section, Cor. 1, and the elementary theory of quadratic forms over the field with 2 elements.

Corollary 4. Let $L$ be a totally symmetric ample invertible sheaf of separable type $\delta$. Then $H(L)$ contains all points of order 2 on $X$, hence if $\delta=\left(d_{1}, \ldots, d_{r}\right)$, then $r=\operatorname{dim} X$ and all $d_{i}$ are even. 
Proof. If $L$ is of type $\delta=\left(d_{1}, \ldots, d_{r}\right)$, then $r \leqq \operatorname{dim} X$ since $H(\delta)$ is isomorphic to a subgroup of $X$. Suppose you add some extra $d$ 's equal to 1 to make a total of $\operatorname{dim} X$ of them. Then by Proposition 4 and the known structure of the group of points on $X$ of finite order, $L^{2}$ is of type $2 \delta$. But if $L$ is totally symmetric, then $e^{\left(L^{2}\right)} \equiv 1$ on points of order 2 . Since $e^{\left(L^{2}\right)}$ is non-degenerate on $H\left(L^{2}\right)$, this means that every point of order 2 is twice a point of order 4 in $H\left(L^{2}\right)$. Therefore $H\left(L^{2}\right)$ contains the whole group $X_{4}$ of points of order 4 on $X$; hence $H(L)$ contains $X_{2}$. Q.E.D.

The last point that I want to deal with in this section is to give a normal form to the pair of groups $\mathscr{G}(L), \mathscr{G}\left(L^{2}\right)$ and the maps between them.

Definition. Let $\delta=\left(d_{1}, d_{2}, \ldots, d_{k}\right)$ be a set of elementary divisors: i.e., $d_{i} \in Z, d_{i+1} \mid d_{i}, d_{i} \geqq 1$. (N.b., we allow some l's at the end.) Then $2 \delta$ is the sequence of divisors $\left(2 d_{1}, 2 d_{2}, \ldots, 2 d_{k}\right)$. We shall always regard $K(\delta)=\oplus Z / d_{i} Z$ as a subgroup of $K(2 \delta)=\oplus Z / 2 d_{i} Z$ under the map $\left(a_{1}, \ldots, a_{k}\right) \mapsto\left(2 a_{1}, \ldots, 2 a_{k}\right)$. Then the dual $\widehat{K(\delta)}$ is naturally a quotient of $\widehat{K(2 \delta)}$. If $l \in \widehat{K(2 \delta)}$, then $\bar{l}$ will denote its natural image in $\widehat{K(\delta)}$. However, if $l \in \widehat{K(\delta)}$, then there is a unique $l^{\prime} \in \widehat{K(2 \delta)}$ such that

$$
l^{\prime}(x)=l(2 x)
$$

for all $x \in K(2 \delta)$. This defines an injection of $\widehat{K(\delta)}$ into $\widehat{K(2 \delta)}$ that we denote $2 *$, i.e., we set

$$
l^{\prime}=2 * l \text {. }
$$

Definition. $E_{2}: \mathscr{G}(\delta) \rightarrow \mathscr{G}(2 \delta)$ is the map

$$
E_{2}((\alpha, x, l))=\left(\alpha^{2}, x, 2 * l\right) .
$$

Definition. $D_{n}: \mathscr{G}(\delta) \rightarrow \mathscr{G}(\delta)$ is the map

$$
D_{n}((\alpha, x, l))=\left(\alpha^{n^{2}}, n x, n l\right) \text {. }
$$

Definition. $H_{2}: \mathscr{G}(2 \delta) \rightarrow \mathscr{G}(\delta)$ is the map

$$
H_{2}((\alpha, x, l))=\left(\alpha^{2}, 2 x, \bar{l}\right) \text {. }
$$

One checks immediately that $E_{2}, H_{2}$ and $D_{n}$ are all homomorphisms. Note also that

$$
\begin{gathered}
E_{2} \circ H_{2}=D_{2} \text { for } \mathscr{G}(2 \delta) \\
H_{2} \circ E_{2}=D_{2} \text { for } \mathscr{G}(\delta) \\
D_{n}(z)=z^{\frac{n^{2}+n}{2}} \cdot D_{-1}(z)^{\frac{n^{2}-n}{2}}, \text { all } z \in \mathscr{G}(\delta)
\end{gathered}
$$

just as was the case with $\varepsilon, \eta$ and $\delta$. 
Definition. Let $L$ be a totally symmetric ample invertible sheaf of separable type $\delta$. A $\vartheta$-structure

$$
f: \mathscr{G}(L) \stackrel{\sim}{\longrightarrow} \mathscr{G}(\delta)
$$

will be called symmetric if $f \circ \delta_{-1}=D_{-1} \circ f$. A pair of $\vartheta$-structures $f_{1}$ for $L$ and $f_{2}$ for $L^{2}$ will be called a symmetric $\vartheta$-structure for $\left(L, L^{2}\right)$ or a compatible pair of symmetric $\vartheta$-structures if

An isomorphism

$$
\begin{aligned}
& f_{2} \circ \varepsilon_{2}=E_{2} \circ f_{1} \\
& f_{1} \circ \eta_{2}=H_{2} \circ f_{2} .
\end{aligned}
$$

$$
g: H(L) \stackrel{\sim}{\longrightarrow} H(\delta)
$$

will be called symplectic if, for all $z_{1}, z_{2} \in H(L), g\left(z_{1}\right)=\left(x_{1}, l_{1}\right), g\left(z_{2}\right)=$ $\left(x_{2}, l_{2}\right)$, then

$$
e^{(L)}\left(z_{1}, z_{2}\right)=l_{2}\left(x_{1}\right) \cdot l_{1}\left(x_{2}\right)^{-1} \text {. }
$$

Note that if $f: \mathscr{G}(L) \rightarrow \mathscr{G}(\delta)$ is any $\vartheta$-structure, then the induced isomorphism of $H(L)$ and $H(\delta)$ is symplectic (check commutators!). Summarizing the situation so far, we can draw up a chart of the possible "markings" with which we can endow $L, L^{2}$ and $L^{4}$. The solid arrows indicate that a structure of one type automatically induces one of the other. (Ignore dotted arrows.)

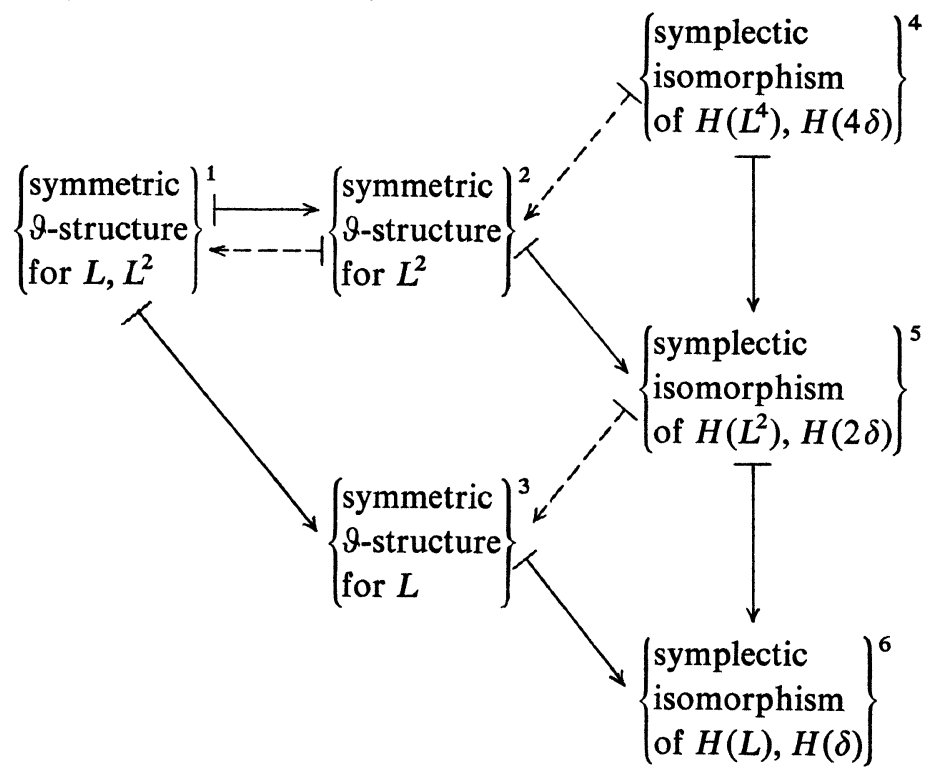

Remark 1 . Actually, every symmetric $\vartheta$-structure for $L^{2}$ extends to a symmetric $\vartheta$-structure for $L, L^{2}$. Thus the arrow from box 1 to box 2 is a bijection. 
Proof. Let $f_{2}: \mathscr{G}\left(L^{2}\right) \rightarrow \mathscr{G}(2 \delta)$ be the given structure for $L^{2}$. Via $\eta_{2}, \mathscr{G}(L)$ is identified with the quotient of $\mathscr{G}\left(L^{2}\right)$ by its points of order 2 [Corollary 2 to Prop. 6]. Since all the $d_{i}$ are even, $\mathscr{G}(\delta)$ is identified with the quotient of $\mathscr{G}(2 \delta)$ by its points of order 2 via $H_{2}$. Therefore, $f_{2}$ induces a unique isomorphism $f_{1}$ of these quotients, i.e.,

$$
f_{1}: \mathscr{G}(L) \stackrel{\sim}{\longrightarrow} \mathscr{G}(\delta)
$$

such that $H_{2} \circ f_{2}=f_{1} \circ \eta_{2}$. To check that $f_{2} \circ \varepsilon_{2}=E_{2} \circ f_{1}$, it suffices to consider this for elements of the form $\eta_{2}(z)$. But then

$$
\begin{aligned}
f_{2}\left(\varepsilon_{2}\left(\eta_{2}(z)\right)\right) & =f_{2}\left(\delta_{2}(z)\right) \\
& =f_{2}\left(z^{3} \cdot \delta_{-1}(z)\right) \\
& =f_{2}(z)^{3} \cdot D_{-1}\left[f_{2}(z)\right] \\
& =D_{2} f_{2}(z) \\
& =E_{2}\left(H_{2}\left(f_{2}(z)\right)\right) \\
& =E_{2}\left(f_{1}\left(\eta_{2}(z)\right)\right) \cdot \quad \text { Q.E.D. }
\end{aligned}
$$

Remark 2. Every symplectic isomorphism $g: H(L) \stackrel{\sim}{\longrightarrow} H(\delta)$ is induced by a symmetric $\vartheta$-structure on $L$ : i.e., the arrow from box 3 to box 6 is surjective [also that from box 2 to box 5].

Proof. We saw in $\S 1$ that every symplectic isomorphism is induced from some $\vartheta$-structure: i.e., let $\bar{K}_{1}=g^{-1}[K(\delta)], \bar{K}_{2}=g^{-1}[\widehat{K(\delta)}]$; lift $\bar{K}_{1}, \bar{K}_{2}$ to level subgroups $K_{1}, K_{2} \subset \mathscr{G}(L)$; construct $f: \mathscr{G}(L) \rightarrow \mathscr{G}(\delta)$ by mapping $K_{1}$ into the points $(1, x, 0), K_{2}$ into the points $(1,0, l)$ and extending multiplicatively.

The problem is to alter $f$ to make it symmetric without altering the induced map $g$. For all $\sigma \in \operatorname{Hom}\left[H(\delta), k^{*}\right]$, define the automorphism $F_{\sigma}$ of $\mathscr{G}(\delta)$ by:

$$
F_{\sigma}((\alpha, x, l))=(\alpha \cdot \sigma(x, l), x, l) .
$$

Then we seek $\sigma$ such that $F_{\sigma} \circ f$ is symmetric. Let $D_{-1}^{*}=f \circ \delta_{-1} \circ f^{-1}$. Then $D_{-1}^{*}$ is an automorphism of $\mathscr{G}(\delta)$ of order 2 , the identity on $k^{*}$, and -1 on $H(\delta)$. Therefore it is of the form:

$$
D_{-1}^{*}((\alpha, x, l))=(\alpha \cdot \tau(x, l),-x,-l)
$$

where $\tau$ is a homomorphism. If we replace $f$ by $F_{\sigma} \circ f, D_{-1}^{*}$ is replaced by $F_{\sigma} \circ D_{-1}^{*} \circ F_{\sigma}^{-1}$. Hence we must choose $\sigma$ such that

$$
\tau(x, l)=\sigma(x, l)^{2}
$$

all $(x, l) \in H(\delta)$. This can be done if $\tau \equiv 1$ on points of order 2; i.e., $D_{-1}^{*}(z)=z$ for all $z$ of order 2. This follows from Prop. 3 since $L$ is totally symmetric. 
Remark 3. Given a symplectic isomorphism $g_{2}: H\left(L^{2}\right) \stackrel{\sim}{\longrightarrow} H(2 \delta)$, there is a unique symmetric $\vartheta$-structure $f_{1}: \mathscr{G}(L) \stackrel{\sim}{\longrightarrow} \mathscr{G}(\delta)$ such that both come from some common symmetric $\vartheta$-structure on $L^{2}$, i.e., there is a unique dotted arrow from box 5 to box 3 giving us a commutative diagram [similarly there is a natural arrow from box 4 to box 2].

Proof. Lift $g_{2}$ to a symmetric $f_{2}: \mathscr{G}\left(L^{2}\right) \stackrel{\sim}{\longrightarrow} \mathscr{G}(2 \delta) . f_{2}$ then induces an $f_{1}$ as in Remark 1 . If $f_{2}^{\prime}$ also lifts $g_{2}$, then $f_{2}^{\prime}=F_{\sigma} \circ f_{2}$, where $\sigma \in \operatorname{Hom}\left[K(2 \delta), k^{*}\right]$. Since $f_{2}$ and $f_{2}^{\prime}$ are symmetric, $F_{\sigma} \circ D_{-1}=D_{-1} \circ F_{\sigma}$. This implies that $\sigma(z)= \pm 1$, for all $z \in K(2 \delta)$. Therefore $H_{2}\left(f_{2}(z)\right)=$ $H_{2}\left(f_{2}^{\prime}(z)\right)$ for all $z \in \mathscr{G}(2 \delta)$. Therefore $f_{2}$ and $f_{2}^{\prime}$ induce the same $f_{1}$. Q.E.D.

We need one computation here:

Given

Assume

$$
\begin{aligned}
& g_{2}: \quad H\left(L^{2}\right) \rightarrow H(2 \delta) \text { symplectic } \\
& \Delta_{1}: \quad K(2 \delta) \rightarrow \text { pts of order } 2 \text { in } \widehat{K(2 \delta)} \\
& \Delta_{2}: \widehat{K(2 \delta)} \rightarrow \text { pts of order } 2 \text { in } K(2 \delta) .
\end{aligned}
$$

Then:

$$
\begin{aligned}
& \Delta_{1} x(y)=\Delta_{1} y(x), \quad \text { all } x, y \in K(2 \delta), \\
& l\left(\Delta_{2} m\right)=m\left(\Delta_{2} l\right),
\end{aligned}
$$

$$
g_{2}^{\prime}=\left[1+\left(\begin{array}{cc}
0 & \Delta_{1} \\
\Delta_{2} & 0
\end{array}\right)\right] \circ g_{2}
$$

is also a symplectic isomorphism.

Under the correspondence of Remark 3, let $g_{2} \mapsto f_{1}, g_{2}^{\prime} \mapsto f_{1}^{\prime}$ where $f_{1}, f_{1}^{\prime}$ are symmetric $\vartheta$-structures on $L$. Then $f_{1}^{\prime}=F_{\sigma} \circ f_{1}$, where $\sigma \in \operatorname{Hom}\left(K(\delta), k^{*}\right)$ is given by:

$$
\sigma((2 x, \bar{l}))=\Delta_{1} x(x) \cdot l\left(\Delta_{2} l\right), \text { all }(x, l) \in K(2 \delta) .
$$

This is a tedious but straightforward verification. With it we can prove:

Remark 4. Every symmetric 9 -structure for $L$ is induced by a symplectic isomorphism of $H\left(L^{2}\right), H(2 \delta)$; thus all arrows in our diagram are surjective.

Proof. Let $f_{1}: \mathscr{G}(L) \sim \mathscr{\longrightarrow}(\delta)$ be the symmetric $\vartheta$-structure. $f_{1}$ induces a symplectic $g_{1}: H(L) \stackrel{\sim}{\longrightarrow} H(\delta)$, which lifts to a symplectic $g_{2}: H\left(L^{2}\right) \stackrel{\sim}{\longrightarrow}$ $H(2 \delta)$. As in remark $3, g_{2}$ induces a symmetric $f_{1}^{\prime}: \mathscr{G}(L) \rightarrow \mathscr{G}(\delta)$. Then $f_{1}^{\prime}=F_{\sigma} \circ f_{1}$ for some $\sigma \in \operatorname{Hom}\left(K(\delta), k^{*}\right)$. Since $f_{1}$ and $f_{1}^{\prime}$ are both symmetric, $F_{\sigma}$ and $D_{-1}$ commute, hence $\operatorname{Im}(\sigma) \subset\{ \pm 1\}$. But then using the 
computation we have just made, it follows that we can "correct" $g_{2}$, not altering its values on $H(L)$, so that $f_{1}^{\prime}$ is changed to $f_{1}$. Q.E.D.

In conclusion, a symmetric $\vartheta$-structure on $X$ can be considered as something intermediate between a labeling of all the points in $H\left(L^{2}\right)$, or of only the points in $H(L)$. Moreover, we have proven:

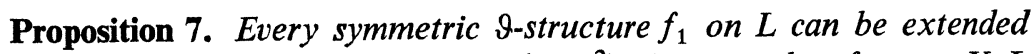
to a symmetric $\vartheta$-structure $\left(f_{1}, f_{2}\right)$ on $\left(L, L^{2}\right)$. In particular, for any $X, L$ as above, the pair of groups $\mathscr{G}(L), \mathscr{G}\left(L^{2}\right)$ and the pairs of maps $\varepsilon_{2}, \eta_{2}$ is isomorphic to $\mathscr{G}(\delta), \mathscr{G}(2 \delta), E_{2}, H_{2}$.

\section{§3. The Addition Formula}

The most important application of the last theorem of $\S 1$ is to give an explicit description of the group law on an abelian variety $X$ in terms of its canonical projective embedding. To express the group law algebraically, the first idea one might have would be to make the homogeneous coordinate ring:

$$
R=\bigoplus_{n=0}^{\infty} \Gamma\left(X, L^{n}\right)
$$

into a Hopf algebra, (here $L$ is a very ample invertible sheaf on $X$ ). Thus if $\mu: X \times X \rightarrow X$ is the group law, $R$ would be a Hopf algebra if $\mu^{*} L \cong$ $p_{1}^{*} L \otimes p_{2}^{*} L$. However $\mu^{*}(L)$ is not isomorphic on $X \times X$ to $p_{1}^{*} L \otimes p_{2}^{*} L$ or to any other sheaf on $X \times X$ which is directly built up out of $L$ by means of the projections of $X \times X$ onto $X$. So while $\mu^{*}$ induces a map from $\Gamma(X, L)$ to $\Gamma\left(X \times X, \mu^{*} L\right), \Gamma\left(X \times X, \mu^{*} L\right)$ cannot be directly related back with $\Gamma(X, L)$. What we can do, however, is to describe the isogeny:

$$
\xi: \quad X \times X \rightarrow X \times X
$$

such that $\xi(x, y)=(x+y, x-y)$, for all closed points $x, y$ in $X$. In fact, concerning $\xi$ we have:

Proposition 1. Let $\imath: X \rightarrow X$ be the inverse morphism on $X$. Then for all invertible sheaves $L$ on $X$ such that $\imath^{*} L \cong L$, if $M$ is the invertible sheaf $p_{1}^{*} L \otimes p_{2}^{*} L$ on $X \times X$, then:

$$
\xi^{*}(M) \cong M^{2}
$$

Proof. By the see-saw principle, it suffices to check that $\xi^{*}(M)$ and $M^{2}$ are isomorphic when restricted to the sub-schemes $X \times\{a\}$ and $\{e\} \times X$ of $X \times X$, for all closed points $a \in X$ (here $e$ is the identity point on $X$ ). Let

$$
s_{a}^{(i)}: \quad X \rightarrow X \times X, \quad i=1,2, \quad a \in X_{k}
$$


be the morphisms such that $s_{a}^{(1)}(x)=(x, a), s_{a}^{(2)}(x)=(a, x)$ for all closed points $x \in X$. Then:

$$
\begin{aligned}
\left(s_{a}^{(i)}\right)^{*}\left(M^{2}\right) & =\left(s_{a}^{(i)}\right)^{*}\left(p_{1}^{*} L^{2} \otimes p_{2}^{*} L^{2}\right) \\
& =\left(p_{1} \circ s_{a}^{(i)}\right)^{*} L^{2} \otimes\left(p_{2} \circ s_{a}^{(i)}\right)^{*} L^{2} .
\end{aligned}
$$

Since $p_{i} \circ s_{a}^{(i)}=$ identity on $X$, and if $i \neq j$, then $p_{j} \circ s_{a}^{(i)}$ maps $X$ to the point $a$, we find:

On the other hand:

$$
\left(s_{a}^{(i)}\right)^{*}\left(M^{2}\right) \cong L^{2} .
$$

$$
\begin{aligned}
\left(s_{a}^{(1)}\right)^{*} \xi^{*}(M) & =\left(s_{a}^{(1)}\right)^{*} \xi^{*}\left(p_{1}^{*} L \otimes p_{2}^{*} L\right) \\
& =\left[p_{1} \circ \xi \circ s_{a}^{(1)}\right]^{*} L \otimes\left[p_{2} \circ \xi \circ s_{a}^{(2)}\right]^{*} L \\
& =T_{a}^{*} L \otimes T_{-a}^{*} L \\
& \cong L^{2} .
\end{aligned}
$$

(The last isomorphism comes from the theorem of the square.) Finally:

$$
\begin{aligned}
\left(s_{e}^{(2)}\right)^{*} \xi^{*}(M) & =\left(s_{e}^{(2)}\right)^{*} \xi^{*}\left(p_{1}^{*} L \otimes p_{2}^{*} L\right) \\
& =\left[p_{1} \circ \xi \circ s_{e}^{(2)}\right]^{*} L \otimes\left[p_{2} \circ \xi \circ s_{e}^{(2)}\right]^{*} L \\
& =L \otimes \imath^{*} L \\
& \cong L^{2} .
\end{aligned}
$$

Therefore $\xi^{*} M$ and $M^{2}$ have isomorphic restrictions on all the required subschemes, hence are isomorphic. Q.E.D.

Now, by the Künneth formula:

$$
\Gamma\left(X \times X, M^{r}\right) \cong \Gamma\left(X, L^{r}\right) \otimes \Gamma\left(X, L^{r}\right) .
$$

Therefore, $\xi$ induces a map $\varphi$ :

$$
\begin{aligned}
& \Gamma(X, L) \otimes \Gamma(X, L) \cong \Gamma(X \times X, M) \\
& \begin{array}{r}
\text { Künneth } \\
\stackrel{\xi^{*}}{\Gamma\left(X \times X, \xi^{*} M\right)}
\end{array} \\
& \text { 2\| } \\
& \Gamma\left(X \times X, M^{2}\right) \underset{\text { Kunneth }}{\cong} \Gamma\left(X, L^{2}\right) \otimes \Gamma\left(X, L^{2}\right) .
\end{aligned}
$$

Moreover, if $L$ is very ample on $X$, then $M$ is very ample on $X \times X$ and this $\operatorname{map} \varphi$ is sufficient to determine the morphism $\xi$. We want to apply 22* 
Theorem 4 of $\S 1$ to determine $\varphi$, and hence, in terms of canonical bases of $\Gamma(X, L)$ and $\Gamma\left(X, L^{2}\right)$, to give a canonical matrix representing $\varphi$ (independent of the moduli of $X$ !). Unfortunately, we have to make a fundamental restriction at this point:

Proposition. If $\operatorname{dim} X=g, \operatorname{deg}(\xi)=2^{2 \mathrm{~g}} ; \xi$ is separable if and only if $\operatorname{char}(k) \neq 2$.

Proof. It is convenient to use the language of schemes to prove this: let $z=\left(x_{1}, x_{2}\right)$ be an $R$-valued point of $X \times X$, for some $k$-algebra $R$. Then $z$ is in the kernel of $\xi$ if and only if $x_{1}+x_{2}=x_{1}-x_{2}=e$ : i.e., $x_{1}=x_{2}$ and $2 x_{1}=e$. Therefore the map taking $z$ to $x_{1}$ defines an isomorphism of the kernel of $\xi$ with the kernel of

$$
2 \delta: \quad X \rightarrow X
$$

(multiplication by 2). But in general the degree of $k \delta$ (multiplication by $k$ ) is $k^{2 g}$, hence $\operatorname{deg}(\xi)=2^{2 g}$. If char $(k) \neq 2$, then $\xi$ is clearly separable. If char $(k)=2$, then it is well known that $2 \delta$ is not separable, hence its kernel is not reduced, hence the kernel of $\xi$ is not reduced, hence $\xi$ is not separable. Q.E.D.

From now on, we assume char $(k) \neq 2$. Then if $L$ is ample of separable type, so are $L^{2}, M$, and $M^{2}$ and the theory of $\S 1$ is applicable.

Assume moreover that $L$ is actually totally symmetric: hence so are $L^{2}, M$, and $M^{2}$. Now choose a symmetric $\vartheta$-structure on $\left(L, L^{2}\right)$ i.e., isomorphism $f_{1}: \mathscr{G}(L) \stackrel{\sim}{\longrightarrow} \mathscr{G}(\delta)$ and $f_{2}: \mathscr{G}\left(L^{2}\right) \stackrel{\sim}{\longrightarrow} \mathscr{G}(2 \delta)$ as in Proposition 7 of $\S 2$. Recall that we showed in $\S 1$ that these isomorphisms induce isomorphisms:

$$
\begin{aligned}
& \beta_{1}: \quad \Gamma(X, L) \stackrel{\longrightarrow}{\longrightarrow}(\delta)=\left\{\begin{array}{l}
\text { vector space of } k \text {-valued } \\
\text { functions on } K(\delta)
\end{array}\right\} \\
& \beta_{2}: \quad \Gamma\left(X, L^{2}\right) \stackrel{\sim}{\longrightarrow}(2 \delta)=\left\{\begin{array}{l}
\text { vector space of } k \text {-valued } \\
\text { functions on } K(2 \delta)
\end{array}\right\}
\end{aligned}
$$

unique up to scalar multiples. Choose some pair of $\beta$ 's. How about $\Gamma(X, M)$ and $\Gamma\left(X, M^{2}\right)$ ? $\beta_{1}$ and $\beta_{2}$ immediately induce:

$$
\begin{aligned}
\Gamma(X \times X, M) \cong \Gamma(X, L) \otimes \Gamma(X, L) \\
\left\{\begin{array}{l}
\text { fcns. on } \\
K(\delta)
\end{array}\right\} \otimes\left\{\begin{array}{l}
\text { fcns. on } \\
K(\delta)
\end{array}\right\} \cong\left\{\begin{array}{l}
\text { fcns. on } \\
K(\delta) \times K(\delta)
\end{array}\right\}
\end{aligned}
$$


and similarly

$$
\Gamma\left(X \times X, M^{2}\right) \underset{\beta_{2}^{(2)}}{\cong}\left\{\begin{array}{l}
\text { functions on } \\
K(2 \delta) \times K(2 \delta)
\end{array}\right\} .
$$

Actually, these maps are of the same type as $\beta$ : i.e., they put the usual group action in standard form. Note:

Lemma 1. Let $X$ and $Y$ be abelian varieties, and let $L, M$ be ample invertible sheaves of separable type on $X$ and $Y$. Then

$$
\mathscr{G}\left(p_{1}^{*} L \otimes p_{2}^{*} M\right) \cong \mathscr{G}(L) \times \mathscr{G}(M) /\left\{\left(\alpha, \alpha^{-1}\right) \mid \alpha \in k^{*}\right\} .
$$

Proof. Just note how this isomorphism is set up. Given $x \in H(L)$, $y \in H(M)$ and

we obtain

$$
\varphi: \quad L \stackrel{\sim}{\longrightarrow} T_{x}^{*} L, \quad \psi: \quad M \stackrel{\sim}{\longrightarrow} T_{y}^{*} M,
$$

$$
p_{1}^{*} \varphi \otimes p_{2}^{*} \psi: \quad p_{1}^{*} L \otimes p_{2}^{*} M \stackrel{\sim}{\longrightarrow} T_{(x, y)}^{*}\left[p_{1}^{*} L \otimes p_{2}^{*} M\right] .
$$

The lemma is now readily checked. Q.E.D.

Now returning to our original set-up, it follows from the lemma that the isomorphism

$$
f_{1}: \mathscr{G}(L) \stackrel{\sim}{\longrightarrow} \mathscr{G}(\delta)=\left\{(\alpha, x, l) \mid \alpha \in k^{*}, x \in K(\delta), l \in \widehat{K(\delta)}\right\}
$$

induces an isomorphism:

$f_{1}^{(2)}: \mathscr{G}(M) \stackrel{\sim}{\longrightarrow} \mathscr{G}(\delta)^{(2)}=\left\{\left(\alpha, x_{1}, x_{2}, l_{1}, l_{2}\right) \mid \alpha \in k^{*}, x_{i} \in K(\delta), l_{i} \in \widehat{K(\delta)}\right\}$.

Here the multiplication in $\mathscr{G}(\delta)^{(2)}$ is given by:

$$
\begin{aligned}
& \left(\alpha, x_{1}, x_{2}, l_{1}, l_{2}\right) \cdot\left(\alpha^{\prime}, x_{1}^{\prime}, x_{2}^{\prime}, l_{1}^{\prime}, l_{2}^{\prime}\right) \\
& \quad=\left(\alpha \cdot \alpha^{\prime} \cdot l_{1}^{\prime}\left(x_{1}\right) \cdot l_{2}^{\prime}\left(x_{2}\right), x_{1}+x_{1}^{\prime}, x_{2}+x_{2}^{\prime}, l_{1}+l_{1}^{\prime}, l_{2}+l_{2}^{\prime}\right) .
\end{aligned}
$$

Now let $\mathscr{G}(\delta)^{(2)}$ act on the vector space of $k$-valued functions on $K(\delta) \times$ $K(\delta)$ - call this vector space $V(\delta)^{(2)}$ - as follows:

$$
\begin{gathered}
z=\left(\alpha, x_{1}, x_{2}, l_{1}, l_{2}\right) \in \mathscr{G}(\delta)^{(2)}, \quad f \in V(\delta)^{(2)}, \\
\left(U_{z} f\right)\left(u_{1}, u_{2}\right)=\alpha l_{1}\left(u_{1}\right) l_{2}\left(u_{2}\right) f\left(u_{1}+x_{1}, u_{2}+x_{2}\right) .
\end{gathered}
$$

Then $\beta_{1}^{(2)}$ is determined, up to scalar multiples, by the readily verified property:

$$
\beta_{1}^{(2)}\left(U_{z} s\right)=U_{f_{1}^{(2)}(z)}\left(\beta_{1}^{(2)} s\right),
$$

for all $z \in \mathscr{G}(M), s \in \Gamma(X \times X, M)$. 
Now $\xi^{*}$ defines the map $\beta_{2}^{(2)} \circ \varphi \circ \beta_{1}^{(2)-1}$ :

$$
\begin{aligned}
& V(\delta)^{(2)}=\left\{\begin{array}{l}
\text { fcns. on } \\
K(\delta) \times K(\delta)
\end{array}\right\} \underset{\beta_{1}^{(2)-1}}{\sim} \underset{\sim}{\sim} \Gamma(X \times X, M) \\
& \Gamma\left(X \times \stackrel{\downarrow}{X}, \xi^{*} M\right) \\
& \Gamma\left(X \times X, M^{2}\right) \underset{\beta_{2}^{(2)}}{\sim}\left\{\begin{array}{l}
\text { fens. on } \\
K(2 \delta) \times K(2 \delta)
\end{array}\right\} \\
& =V(2 \delta)^{(2)} \text {. }
\end{aligned}
$$

Call this transformation $\Omega$.

Fundamental Addition Formula. There is a scalar $\lambda$, such that for all $f \in V(\delta)^{(2)}$

$$
(\Omega f)(x, y)=\left\{\begin{array}{lll}
0 & \text { if } & x+y \notin K(\delta) \\
\lambda \cdot f(x+y, x-y) & \text { if } & x+y \in K(\delta)
\end{array}\right.
$$

for all $x, y \in K(2 \delta)$.

Remark. By suitably normalizing our maps $\beta$, we can always assume $\lambda=1$. In what follows, I will always assume that this has been done.

Proof. This formula is a special case of the general formula given in Theorem 4, § 1 . To see this, let

$$
\mathscr{G}\left(M^{2}\right)^{*}=\left\{\begin{array}{l}
\text { subgroup of } \mathscr{G}\left(M^{2}\right) \text { of elements lying } \\
\text { over points of } X \times X \text { in } \xi^{-1}[H(M)]
\end{array}\right.
$$

and let $\tilde{K} \subset \mathscr{G}\left(M^{2}\right)$ be the descent data associated to $\xi$. Then we have the canonical map (cf. §1):

$$
\tau: \frac{\mathscr{G}\left(M^{2}\right)^{*}}{\tilde{K}} \stackrel{\sim}{\longrightarrow} \mathscr{G}(M) .
$$

First of all, $\mathscr{G}\left(M^{2}\right)^{*}$ goes over via our $\vartheta$-structure to the subgroup of $\mathscr{G}(2 \delta)^{(2)}$ :

$$
\left.\begin{array}{rl}
\mathscr{G}(2 \delta)^{*}=\left\{\left(\alpha, x_{1}, x_{2}, l_{1}, l_{2}\right)\right. & \begin{array}{l}
x_{i} \in K(2 \delta), x_{1}+x_{2} \in K(\delta) \\
l_{i} \in \widehat{K(2 \delta), l_{1}+l_{2}=2 * l}
\end{array} \\
\text { for some } l \in \widehat{K(\delta)}
\end{array}\right\}
$$

In fact, we are given an isomorphism:

$$
\begin{aligned}
& X \times X
\end{aligned}
$$

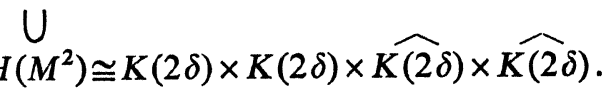


and $\xi$ maps the point $\left(x_{1}, x_{2}, l_{1}, l_{2}\right)$ in the latter group to $\left(x_{1}+x_{2}\right.$, $\left.x_{1}-x_{2}, l_{1}+l_{2}, l_{1}-l_{2}\right)$. Then $\left(\alpha, x_{1}, x_{2}, l_{1}, l_{2}\right)$ is in the group corresponding to $\mathscr{G}\left(M^{2}\right)^{*}$ if and only if $\left(x_{1}+x_{2}, x_{1}-x_{2}, l_{1}+l_{2}, l_{1}-l_{2}\right)$ corresponds to a point of $H(M)$. But this is the same as asking that $x_{1}+x_{2} \in 2 K(2 \delta)$ [then $x_{1}-x_{2}$ is automatically also in $2 K(2 \delta)$ ] and that $l_{1}+l_{2} \in 2 \widehat{K(2 \delta)}$. But $2 K(2 \delta)=K(\delta)$ under our identifications, and $2 \widehat{K(2 \delta)}$ is the group of homomorphisms $2 * l, l \in \widehat{K(\delta)}$.

Now let $T$ be the homomorphism defined by the diagram:

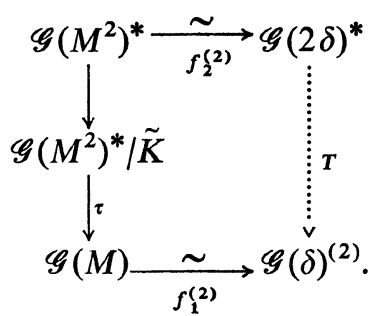

The key fact is contained in the following lemma, (which uses the crucial symmetry assumption):

Lemma 2.

$$
\begin{aligned}
& T\left(\left(\alpha, x_{1}, x_{2}, l_{1}, l_{2}\right)\right)=\left(\alpha, x_{1}+x_{2}, x_{1}-x_{2}, l, k\right) \\
& l_{1}+l_{2}=2 * l, l_{1}-l_{2}=2 * k \text { for } l, k \in \widehat{K(\delta)} .
\end{aligned}
$$

When this is proven, our formula comes directly from Theorem 4 , $\S 1$. In fact, $\widetilde{K}$ must go over via our $\vartheta$-structure to $\operatorname{ker}(T)$, and this, by the lemma, equals

$$
\{(1, x, x, l, l) \mid 2 x=0,2 l=0\} .
$$

Thus, in the notation of Theorem 4,

is given by

$$
\begin{aligned}
K_{1}= & \{(x, x) \in K(2 \delta) \times K(2 \delta) \mid 2 x=0\}, \\
K_{1}^{\perp}= & \{(x, y) \in K(2 \delta) \times K(2 \delta) \mid x+y \in K(\delta)\} \\
& \sigma: \quad K_{1}^{\perp} / K_{1} \stackrel{\sim}{\longrightarrow} K(\delta) \times K(\delta)
\end{aligned}
$$

$$
\sigma((x, y))=(x+y, x-y) .
$$

Then lemma 2 proves that the diagram in Theorem 4 commutes, and our formula can be read off.

Proof of lemma. $T$ is obviously the identity on $k^{*}$, so in order to check the lemma, it will suffice to verify it for elements of the form 
$(1, x, x, l, l)$ and of the form $(1, x,-x, l,-l)$. Namely, if $x_{1}, x_{2} \in K(2 \delta)$ and $x_{1}+x_{2} \in K(\delta)=2 K(2 \delta)$, then there are elements $y, z \in K(2 \delta)$ such that $x_{1}=y+z, x_{2}=y-z$; similarly with $l_{1}, l_{2}$. Therefore $\mathscr{G}(2 \delta)^{*}$ is generated by $k^{*}$ and by elements of the above form.

For elements of the form $(1, x, x, l, l)$, consider the diagram:

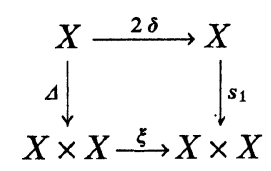

where $\Delta(x)=(x, x)$ is the diagonal, and $s_{1}(x)=(x, 0)$ for closed points $x \in X$. Correspondingly, one has the commutative diagram of sheaf morphisms:

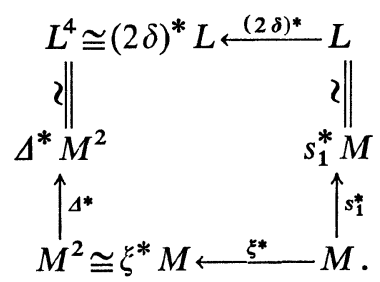

On the level of $H$ 's, this gives maps between subgroups as written out here:

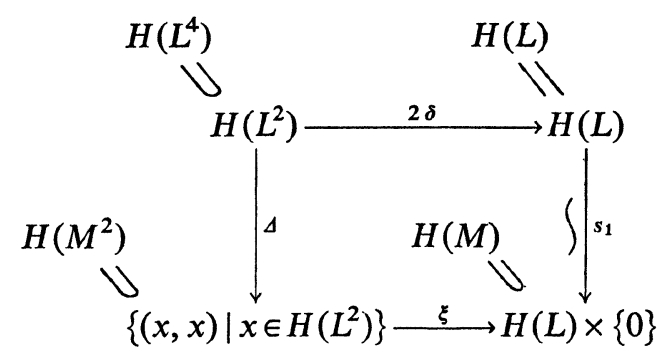

Pulling these subgroups back to the $\mathscr{G}$ 's, and denoting by $\pi$ the projection of each $\mathscr{G}$ onto its $H$, we get the diagram:

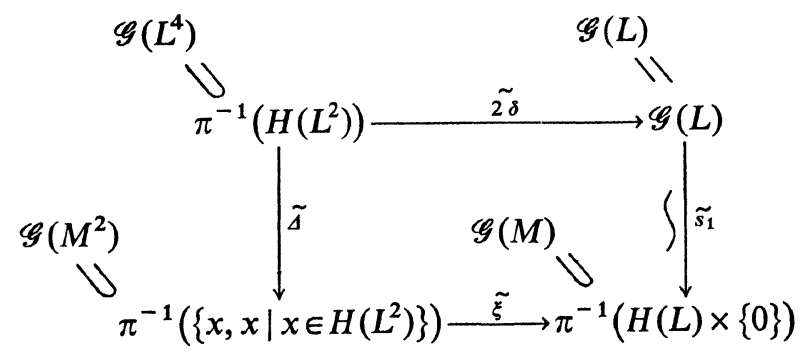


Here the tildas indicate homomorphisms naturally induced by the corresponding homomorphism of abelian varieties. $\tilde{\xi}$ is nothing but the restriction of $\tau$ to the subgroup written out above - which corresponds in $\mathscr{G}(2 \delta)^{*}$ to the group of elements $(\alpha, x, x, l, l)$ : Therefore this diagram is just suited to determine $\tau$, or $T$, on this particular subgroup.

Now start with an element $z=(x, \varphi) \in \mathscr{G}\left(L^{2}\right)$. Then

$$
p_{1}^{*} \varphi \otimes p_{2}^{*} \varphi: \quad M^{2} \stackrel{\sim}{\longrightarrow} T_{(x, x)}^{*} M^{2}
$$

is an element of $\mathscr{G}\left(M^{2}\right)$ in the subgroup $\pi^{-1}\left(\left\{x, x \mid x \in H\left(L^{2}\right)\right\}\right)$. But this isomorphism restricts on the diagonal to the isomorphism

And therefore

$$
\varphi^{2}: M^{4} \stackrel{\sim}{\longrightarrow} T_{x}^{*} M^{4} .
$$

Moreover, if

$$
\left((x, x), p_{1}^{*} \varphi \otimes p_{2}^{*} \varphi\right)=\tilde{\Delta}\left[\left(x, \varphi^{2}\right)\right] .
$$

$$
\psi: L \stackrel{\sim}{\longrightarrow} T_{2 x}^{*} L
$$

is the isomorphism such that $(2 \delta)^{*} \psi=\varphi^{2}$, then

$$
(2 x, \psi)=\tilde{2 \delta}\left[\left(x, \varphi^{2}\right)\right] .
$$

But then, referring back to the definition of $\eta_{2}$, this means that

$$
(2 x, \psi)=\eta_{2}[(x, \varphi)] .
$$

Putting all this together we get a commutative diagram:

$$
\mathscr{G}\left(L^{2}\right) \stackrel{\eta_{2}}{\longrightarrow} \mathscr{G}(L)
$$

where $\alpha[(x, \varphi)]=\left((x, x), p_{1}^{*} \varphi \otimes p_{2}^{*} \varphi\right)$. Now go over to the standard groups $\mathscr{G}(\delta), \mathscr{G}(2 \delta)$, etc. by means of our symmetric system of isomorphisms. We get a diagram:

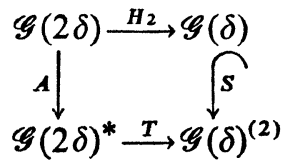

One checks immediately that

and that

$$
A[(\alpha, x, l)]=\left(\alpha^{2}, x, x, l, l\right)
$$

$$
S[(\alpha, x, l)]=(\alpha, x, 0, l, 0) .
$$


Therefore, we calculate:

$$
\begin{aligned}
T[(1, x, x, l, l)] & =S\left[H_{2}[(1, x, l)]\right] \\
& =(1,2 x, 0, \bar{l}, 0)
\end{aligned}
$$

and since $2 * \bar{l}=2 l$, this is the asserted formula.

The proof of the lemma for elements of the form $(1, x,-x, l,-l)$ is very similar, only based on the diagram:

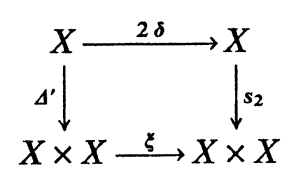

where $\Delta^{\prime}(x)=(x,-x)$, and $s_{2}(x)=(0, x)$ for closed points $x \in X$. This part of the proof is omitted. Q.E.D.

This marvelously simple formula is the basis of the whole theory which follows. The most striking thing about it is that, as promised, it does not involve the moduli of $X$ itself - i.e., it shows, in some sense, that the same addition formula is valid for all abelian varieties. This is something of a cheat, however, as we have only given the map $\varphi$ induced by $\xi$ from $\Gamma(X, L) \otimes \Gamma(X, L) \rightarrow \Gamma\left(X, L^{2}\right) \otimes \Gamma\left(X, L^{2}\right)$. To have the whole story, we need also the canonical map

$$
\Gamma(X, L) \otimes \Gamma(X, L) \rightarrow \Gamma\left(X, L^{2}\right)
$$

given by tensoring a pair of sections. For $L$ sufficiently ample, this will be surjective and hence the addition formula can be written entirely in terms of the one vector space $\Gamma(X, L)$, i.e., in terms of the homogeneous coordinates in one canonical projective embedding. However, the remarkable fact is that this second map is a special case of the first, so that we can pull ourselves up by our bootstraps. In fact, consider the diagram:

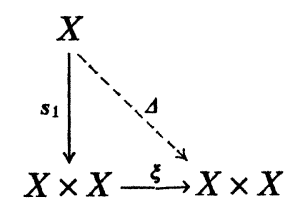

where $\Delta(x)=(x, x), s_{1}(x)=(x, 0)$ for closed points $x \in X$. Then $s_{1}^{*} M^{2} \cong L^{2}$ canonically, and if $t, t^{\prime}$ are two sections of $L$,

$$
s_{1}^{*}\left[\varphi\left(p_{1}^{*} t \otimes p_{2}^{*} t^{\prime}\right)\right]=t \otimes t^{\prime} \in \Gamma\left(X, L^{2}\right) .
$$


Passing over to the representation of these sections by functions on $K$ 's, this gives the diagram:

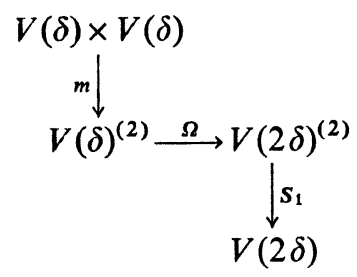

where $S_{1}$ is obtained by carrying over $s_{1}^{*}$, and $m$ takes the pair of functions $f, f^{\prime}$ into the function $g$ of two variables

$$
g(x, y)=f(x) \cdot f^{\prime}(y)
$$

The composition is the "multiplication" of sections of $L$ :

Definition. If $t, t^{\prime} \in \Gamma(X, L)$ and $f=\beta_{1}(t), f^{\prime}=\beta_{1}\left(t^{\prime}\right)$, then let

$$
f * f^{\prime}=\beta_{2}\left(t \otimes t^{\prime}\right) \text {. }
$$

What is $S_{1}$ ? It can be given explicitly if we introduce the null-value function $q_{L^{2}}$ as in $\S 1$. Choose an isomorphism $\lambda_{0}^{(2)}: L(0)^{2} \stackrel{\sim}{\longrightarrow} k-$ actually we already did this implicitly when we identified $s_{1}^{*} M^{2}$ with $L^{2}$. Then there is a natural "evaluation" of sections at 0 :

$$
t \mapsto \lambda_{0}^{(2)}[t(0)], \quad t \in \Gamma\left(X, L^{2}\right) .
$$

In terms of the isomorphism $\beta_{2}$, this gives a unique function $q_{L^{2}}$ on $K(2 \delta)$ such that:

$$
\lambda_{0}^{(2)}[t(0)]=\sum_{z \in K(2 \delta)} \beta_{2} t(z) \cdot q_{L^{2}}(z) .
$$

Now then, say $p_{1}^{*} t \otimes p_{2}^{*} t^{\prime}$ is a given section of $M^{2}$. Then

$$
s_{1}^{*}\left[p_{1}^{*} t \otimes p_{2}^{*} t^{\prime}\right]=\lambda_{0}^{(2)}\left[t^{\prime}(0)\right] \cdot t
$$

(if the identification of $s_{1}^{*} M^{2}$ with $L^{2}$ is chosen properly - otherwise a constant must be put in). In terms of the $V$ 's, this means

if

$$
\begin{gathered}
{\left[S_{1}(g)\right](x)=\left[\sum_{y \in K(2 \delta)} f^{\prime}(y) \cdot q_{L^{2}}(y)\right] \cdot f(x)} \\
g(x, y)=f(x) \cdot f^{\prime}(y) \in V(2 \delta)^{(2)} .
\end{gathered}
$$

Since $S_{1}$ is linear, this means that for any $g \in V(2 \delta)^{(2)}$,

$$
\left[S_{1}(g)\right](x)=\sum_{y \in K(2 \delta)} g(x, y) \cdot q_{L^{2}}(y) .
$$

Putting this and the addition formula together, we conclude: 
Multiplication Formula. If $f, f^{\prime} \in V(\delta)$, then

$$
\left(f * f^{\prime}\right)(x)=\sum_{y \in x+K(\delta)} f(x+y) \cdot f^{\prime}(x-y) \cdot q_{L^{2}}(y) \text { for all } x \in K(2 \delta) .
$$

Another application of the fundamental addition formula is to the duplication and inverse formulas. To obtain the first, we choose symmetric 9-structures now on $L, L^{2}$ and $L^{4}$ such that those for the pair $\left(L, L^{2}\right)$ and for the pair $\left(L^{2}, L^{4}\right)$ are compatible. Then we obtain 3 isomorphisms:

$$
\begin{aligned}
& \beta_{1}: \quad \Gamma(X, L) \stackrel{\sim}{\longrightarrow} V(\delta) \\
& \beta_{2}: \quad \Gamma\left(X, L^{2}\right) \stackrel{\sim}{\longrightarrow}(2 \delta) \\
& \beta_{4}: \quad \Gamma\left(X, L^{4}\right) \stackrel{\sim}{\longrightarrow}(4 \delta) .
\end{aligned}
$$

The endomorphism $2 \delta: X \rightarrow X$ then gives the diagram:

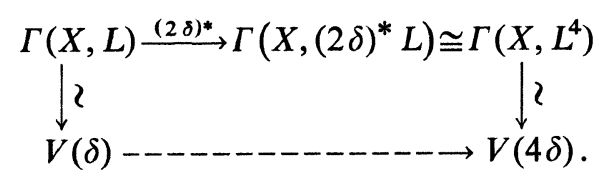

Let the dotted arrow be denoted [2]: this is the duplication homomorphism. Then the result is:

Duplication Formula. If $f \in V(\delta)$, then

$$
\begin{aligned}
& ([2] f)(x)=0 \quad \text { if } \quad x \notin K(2 \delta) \\
& =f(2 x) \text { if } x \in K(2 \delta) \\
& \text { for all } x \in K(4 \delta) \text {. }
\end{aligned}
$$

Proof. We use the fact that $\xi \circ \xi=2 \delta \times 2 \delta$. This implies that if $f_{1}, f_{2} \in V(\delta)$, and if $f_{1} \otimes f_{2}$ is the induced function in $V(\delta)^{(2)}$, then

In other words,

$$
\Omega\left(\Omega f_{1} \otimes f_{2}\right)=[2] f_{1} \otimes[2] f_{2} .
$$

$$
\left(\Omega\left(\Omega f_{1} \otimes f_{2}\right)\right)(x, y)=[2] f_{1}(x) \cdot[2] f_{2}(y)
$$

for all $x, y \in K(4 \delta)$. (Here the first $\Omega$ is for the map $\xi^{*}: M^{2} \rightarrow M^{4}$, and the second $\Omega$ is for the map $\xi^{*}: M \rightarrow M^{2}$.) Substituting the addition formula, the duplication formula follows immediately (at least up to \pm 1 : but this disappears if we correctly normalize the $\beta$ 's). Q.E.D.

The second formula requires only a symmetric $\vartheta$-structure on $L$, hence

$$
\beta_{1}: \quad \Gamma(X, L) \stackrel{\sim}{\longrightarrow}(\delta) .
$$


Using $l$, we get a diagram

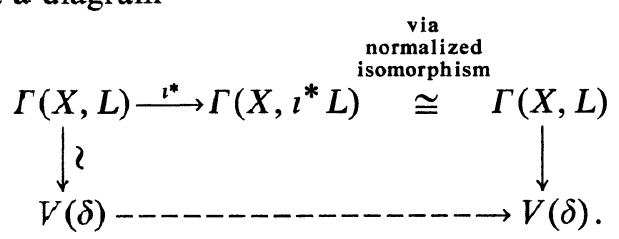

Let the dotted arrow be denoted [-1]. The result is:

Inverse Formula. If $f \in V(\delta)$, then

$$
([-1] f)(x)=f(-x), \text { for all } x \in K(\delta) .
$$

Proof. Up to a constant, the result is quite easy: we use the fact that $\imath=p_{2} \circ \xi \circ s_{2}$, while $1_{X}=p_{1} \circ \xi \circ s_{2}$. Therefore, if $s, t \in \Gamma(X, L)$

$$
s_{2}^{*} \xi^{*}\left[p_{1}^{*} s \otimes p_{2}^{*} t\right] \cong s \otimes \imath^{*} t .
$$

In terms of functions, if $f, f^{\prime} \in V(\delta)$, and $g(x, y)=f(x) \cdot f^{\prime}(y)$ then

$$
\left(f *[-1] f^{\prime}\right)(x)=\sum_{y \in K(2 \delta)}(\Omega g)(y, x) \cdot q_{L^{2}}(y) .
$$

This gives the formula immediately. To get the correct constant (this is meaningful in this case because we are dealing with an automorphism of $\Gamma(X, L))$, note first that $[-1] \circ[-1]=$ identity. Therefore the true formula is either $([-1] f)(x)=f(-x)$ or $-f(-x)$. Since we have used a normalized isomorphism of $\imath^{*} L$ and $L$ to define [-1], it follows that

$$
\sum_{x \in K_{(\delta)}}([-1] f)(x) \cdot q_{L}(x)=\sum_{x \in K(\delta)} f(x) \cdot q_{L}(x) .
$$

In other words, $q_{L}(x)$ is an even function in the first case, and an odd function in the second case. But look at formula (A) below: it is invariant when you interchange $u, v$ and replace $x, y$ by $x,-y$. Therefore $q_{L}$ is even and our formula is correct. Q.E,D.

To conclude this section, we want to show what restrictions these formulas place on the null-functions $q$, and particularly how RiEMANN's theta-relation can be deduced. One word of caution: this relation will not be exactly the same as in the classical case, because our $q$ 's are not the same as the usual theta-null values. But it can be shown that they are related by a non-singular linear transformation with roots of unity as coefficients: so the formulas are trivial modifications of each other. As before, we start by choosing an isomorphism

$$
\lambda_{0}: L(0) \stackrel{\sim}{\longrightarrow} k
$$


This induces

and

$$
\lambda_{0}^{(2)}: L^{2}(0) \stackrel{\sim}{\longrightarrow}
$$

$$
\lambda_{0}^{(4)}: \quad L^{4}(0) \stackrel{\sim}{\longrightarrow} k
$$

$\left(\lambda_{0}^{(2)}\right.$ may as well be the same as the map we used before). Then sections of $L, L^{2}, L^{4}$ have "values" at 0 and we define the $q$ 's via:

Also, $\lambda_{0}$ induces

$$
\begin{array}{cl}
\lambda_{0}[t(0)]=\sum_{z \in K(\delta)} \beta_{1} t(z) \cdot q_{L}(z), & t \in \Gamma(X, L) \\
\lambda_{0}^{(2)}[t(0)]=\sum_{z \in K(2 \delta)} \beta_{2} t(z) \cdot q_{L^{2}}(z), & t \in \Gamma\left(X, L^{2}\right) \\
\lambda_{0}^{(4)}[t(0)]=\sum_{z \in K(4 \delta)} \beta_{4} t(z) \cdot q_{L^{4}}(z), & t \in \Gamma\left(X, L^{4}\right) .
\end{array}
$$

$$
\mu_{0}^{(i)}: \quad M^{i}(0) \stackrel{\sim}{\longrightarrow}, \quad i=1,2,4,
$$

and one checks immediately that:

$$
\mu_{0}^{(i)}[t(0)]=\sum_{x, y \in \mathbf{K}(\delta)} \beta_{i}^{(2)} t(x, y) \cdot q_{L^{i}}(x) \cdot q_{L^{i}}(y)
$$

for all $t \in \Gamma\left(X \times X, M^{i}\right), i=1,2$ or 4 [i.e., check it first for $t$ of the form $p_{1}^{*} t_{1} \otimes p_{2}^{*} t_{2}$ and then use the linearity of both sides]. But there is a constant $\kappa$ such that

$$
\mu_{0}^{(2)}\left[\xi^{*} t(0)\right]=\kappa \cdot \mu_{0}^{(1)}(t(0))
$$

all $t \in \Gamma(X \times X, M)$. And if $\xi^{*}$ is replaced by $\kappa^{-1} \cdot \xi^{*}$, we may as well assume $\kappa=1$. This means that for $f \in V(\delta)^{(2)}$,

$$
\sum_{x, y \in K(\delta)} f(x, y) \cdot q_{L}(x) \cdot q_{L}(y)=\sum_{x, y \in K(2 \delta)} \Omega f(x, y) \cdot q_{L^{2}}(x) \cdot q_{L^{2}}(y) .
$$

Substituting our addition formula into this, and using the fact that this is true for any $f$, we conclude immediately:

(A)

$$
q_{L}(x) \cdot q_{L}(y)=\sum_{\substack{u, v \in K(2 \delta) \\ \text { suth that } \\ u+v=x \\ u=v=y}} q_{L^{2}}(u) \cdot q_{L^{2}}(v) .
$$

Now recall that according to Cor. 4, Prop. 6, § 2,

$$
X_{2} \subset H(L) \subset H\left(L^{2}\right) \subset H\left(L^{4}\right)
$$

where $X_{2}$ is the group of points of order 2 on $X$. Therefore, the 2-torsion subgroups of $K(\delta), K(2 \delta)$, and $K(4 \delta)$ are equal: call this common subgroup $Z_{2}$. Then (A) can be re-written: 
(A') For all $u, v \in K(2 \delta)$ such that $u+v \in K(\delta)$,

$$
q_{L}(u+v) \cdot q_{L}(u-v)=\sum_{\eta \in Z_{2}} q_{L^{2}}(u+\eta) \cdot q_{L^{2}}(v+\eta) .
$$

We can obtain an even simpler relation between $q_{L}$ and $q_{L^{4}}$ either by applying (A) to both of the pairs $\left(L, L^{2}\right)$ and $\left(L^{2}, L^{4}\right)$ and eliminating $q_{L^{2}}$, or by proceeding directly using the duplication formula instead of the addition formula. Following the latter procedure, first note that there is a constant $\kappa$ such that

$$
\lambda_{0}^{(4)}\left[(2 \delta)^{*} t(0)\right]=\kappa \cdot \lambda_{0}(t(0))
$$

for all $t \in \Gamma(X, L)$. If the homomorphism $(2 \delta)^{*}: L \rightarrow L^{4}$ is chosen suitably, the $\kappa$ again disappears. Then the formula goes over on the $V(\delta)$-level to:

$$
\sum_{x \in K(\delta)} f(x) \cdot q_{L}(x)=\sum_{x \in K(4 \delta)}[2] f(x) \cdot q_{L^{4}}(x)
$$

for all $f \in V(\delta)$. Substituting the duplication formula into this, we get out: (B) For all $x \in K(2 \delta)$

$$
q_{L}(2 x)=\sum_{\eta \in Z_{2}} q_{L^{4}}(x+\eta) .
$$

To get a formula involving $q_{L}$ alone, first choose elements $x, y \in K(2 \delta)$ such that $x+y \in K(\delta)$. Then for all homomorphisms $l: Z_{2} \rightarrow\{ \pm 1\}$, we obtain:

$$
\begin{aligned}
\sum_{\zeta \in Z_{2}} l(\zeta) & q_{L}(x+y+\zeta) q_{L}(x-y+\zeta) \\
= & \sum_{\substack{\zeta \in Z_{2} \\
u, v \in K(2 \delta) \\
u+v=x+y+\zeta \\
u-v=x-y+\zeta}} l(\zeta) q_{L^{2}}(u) q_{L^{2}}(v) \\
= & \sum_{\substack{\zeta 1 \\
\zeta_{2} \in Z_{2}}} l\left(\zeta_{1}+\zeta_{2}\right) q_{L^{2}}\left(x+\zeta_{1}\right) q_{L^{2}}\left(y+\zeta_{2}\right) \\
= & {\left[\sum_{\eta \in Z_{2}} l(\eta) q_{L^{2}}(x+\eta)\right] \cdot\left[\sum_{\eta \in Z} l(\eta) q_{L^{2}}(y+\eta)\right] . }
\end{aligned}
$$

Therefore, if $x, y, u, v \in K(2 \delta)$ and $x+y, x+u$, and $x+v \in K(\delta)$, (so that $y+u, y+v, u+v \in K(\delta)$ too), then

$$
\begin{aligned}
& {\left[\sum_{\zeta \in \mathbf{Z}_{2}} l(\zeta) q_{L}(x+y+\zeta) q_{L}(x-y+\zeta)\right] \cdot\left[\sum_{\zeta \in \mathbf{Z}_{2}} l(\zeta) q_{L}(u+v+\zeta) q_{L}(u-v+\zeta)\right]} \\
& =\left[\sum_{\zeta \in \mathbf{Z}_{2}} l(\zeta) q_{L}(x+u+\zeta) q_{L}(x-u+\zeta)\right] \cdot\left[\sum_{\zeta \in Z_{2}} l(\zeta) q_{L}(y+v+\zeta) q_{L}(y-v+\zeta)\right] .
\end{aligned}
$$

Writing these 2 terms together and summing over $l$, we finally obtain:

$$
\text { (C) } \begin{aligned}
\sum_{\zeta \in Z_{2}} q_{L}(x+y+\zeta) q_{L}(x-y+\zeta) q_{L}(u+v+\zeta) q_{L}(u-v+\zeta) \\
=\sum_{\eta \in Z_{2}} q_{L}(x+u+\eta) q_{L}(x-u+\eta) q_{L}(y+v+\eta) q_{L}(y-v+\eta) .
\end{aligned}
$$


To complement this relation, there is the key symmetry property of $q_{L}$ :

$$
q_{L}(-x)=q_{L}(x) \text {. }
$$

This follows as in the proof of the inversion formula. To understand the structure of RIEMANN's theta formula (C) a bit better, note that the variables $x \pm y, u \pm v$ are just 4 arbitrary elements of $K(\delta)$, and that the condition $x+u \in K(\delta)$ amounts to

$$
(x+y)+(x-y)+(u+v)+(u-v) \in 2 K(\delta) .
$$

After observing this, it is easy to rearrange the variables in (C) a bit so as to obtain:

(C') For all $x, y, u, v, z \in K(\delta)$ such that $x+y+u+v=-2 z$,

$$
\begin{aligned}
& \sum_{\eta \in Z_{2}} q_{L}(x+\eta) q_{L}(y+\eta) q_{L}(u+\eta) q_{L}(v+\eta) \\
& \quad=\sum_{\eta \in Z_{2}} q_{L}(x+z+\eta) q_{L}(y+z+\eta) q_{L}(u+z+\eta) q_{L}(v+z+\eta) .
\end{aligned}
$$

We shall give some examples of this in $\S 5$.

The more familiar form of RIEMANN's theta formula is obtained by making a partial Fourier transformation of the function $q_{L}$. Suppose $Z$ is a subgroup of $K(\delta)$ such that:

$$
Z_{2} \subset Z \subset 2 K(\delta) .
$$

Let $H=K(\delta) \times \hat{Z}$, and define a function $\vartheta$ on $H$ by

$$
\vartheta(x)=\sum_{\eta \in \mathbf{Z}} l(\eta) q_{L}(a+\eta)
$$

if $x=(a, l) . \vartheta$ has an obvious periodicity with respect to $Z \times\{0\}$; hence $\vartheta$ is "essentially" a function of $(K(\delta) / Z) \times \hat{Z}$. Let

$$
\begin{aligned}
H_{2} & =\frac{1}{2} Z \times(\hat{Z})_{2} \\
& =\left\{x \in H \mid \begin{array}{c}
x \text { is 2-torsion modulo } \\
Z \times\{0\}
\end{array}\right\} .
\end{aligned}
$$

(C') For all $x, y, u, v, z \in H$ such that $x+y+u+v=-2 z$,

$$
\begin{aligned}
& \vartheta(x) \vartheta(y) \vartheta(u) \vartheta(v) \\
& =\frac{1}{\#\left(\frac{1}{2} Z\right)} \sum_{\xi \in H_{2}} A_{\xi} \cdot \vartheta(x+z+\xi) \vartheta(y+z+\xi) \vartheta(u+z+\xi) \vartheta(v+z+\xi),
\end{aligned}
$$

where if $\xi=(\zeta, m)$ and $z=(e, r), A_{\xi}=(r+m)(2 \zeta)$. 
To obtain $\left(\mathrm{C}^{\prime \prime}\right)$ from $\left(\mathrm{C}^{\prime}\right)$, write the left-hand side out, assuming that

We get

$$
\begin{aligned}
& x=(a, l) \\
& y=(b, k) \\
& u=(c, p) \\
& v=(d, q) \\
& z=(e, r) .
\end{aligned}
$$

$\sum l\left(\eta_{1}\right) k\left(\eta_{2}\right) p\left(\eta_{3}\right) q\left(\eta_{4}\right) q_{L}\left(a+\eta_{1}\right) q_{L}\left(b+\eta_{2}\right) q_{L}\left(c+\eta_{3}\right) q_{L}\left(d+\eta_{4}\right)$. Since $l+k+p+q=-2 r$, this character kills $Z_{2}$. Therefore, the above sum decomposes into a sum of terms of the type

$$
\sum_{\eta \in Z_{2}} q_{L}\left(a^{\prime}+\eta\right) q_{L}\left(b^{\prime}+\eta\right) q_{L}\left(c^{\prime}+\eta\right) q_{L}\left(d^{\prime}+\eta\right)
$$

all of which can be rewritten using $\left(\mathrm{C}^{\prime}\right)$. We get

$$
\begin{aligned}
\frac{1}{2^{g}} \sum_{\substack{\eta_{1}, \ldots, \eta_{4} \in Z \\
\zeta \in \frac{2}{2} Z}} l\left(\eta_{1}\right) k\left(\eta_{2}\right) p\left(\eta_{3}\right) q\left(\eta_{4}\right) & \cdot q_{L}\left(a+e+\zeta+\eta_{1}\right) \cdot q_{L}\left(b+e+\zeta+\eta_{2}\right) \times \\
\eta_{1}+\eta_{2}+\eta_{3}+\eta_{4}=-2 \zeta & \times q_{L}\left(c+e+\zeta+\eta_{3}\right) \cdot q_{L}\left(d+e+\zeta+\eta_{4}\right)
\end{aligned}
$$

which is the same as:

$$
\begin{aligned}
& \frac{1}{2^{g} \cdot \# Z} \sum_{\eta_{1}, \eta_{2}, \eta_{3}, \eta_{4} \in Z} m\left(\eta_{1}+\eta_{2}+\eta_{3}+\eta_{4}+2 \zeta\right) \cdot l\left(\eta_{1}\right) \cdot k\left(\eta_{2}\right) \cdot p\left(\eta_{3}\right) \cdot q\left(\eta_{4}\right) \times
\end{aligned}
$$

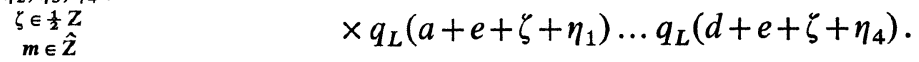

But for fixed $m$, this sum is zero unless $2 m+l+k+p+q=0$, i.e., $m \in r+(\hat{Z})_{2}$. Therefore, the sum works out to be

$$
\begin{aligned}
& \frac{1}{2^{g} \cdot \# Z} \sum_{m \in(\hat{\mathbf{Z}})_{2}}(l+r+m)\left(\eta_{1}\right) \cdot q_{L}\left(a+e+\zeta+\eta_{1}\right) \times
\end{aligned}
$$

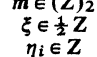

$$
\begin{aligned}
& \times(k+r+m)\left(\eta_{2}\right) \cdot q_{L}\left(b+e+\zeta+\eta_{2}\right) \cdot(p+r+m)\left(\eta_{3}\right) \cdot q_{L}\left(c+e+\zeta+\eta_{3}\right) \times \\
& \times(q+r+m)\left(\eta_{4}\right) \cdot q_{L}\left(d+e+\zeta+\eta_{4}\right) \cdot(r+m)(2 \zeta) .
\end{aligned}
$$

This is exactly the right-hand side of $\left(\mathrm{C}^{\prime \prime}\right)$.

We omit the proof that $\left(\mathrm{C}^{\prime \prime}\right) \Rightarrow\left(\mathrm{C}^{\prime}\right)$ which is similar.

23 Invent. math., Bd. 1 


\section{§4. Structure of the Homogeneous Coordinate Ring}

Let $X$ be an abelian variety, and let $L$ be a totally symmetric ample invertible sheaf of separable type. Of course, char $(k) \neq 2$. In this section we shall study

$$
R=\bigoplus_{n=0}^{\infty} \Gamma\left(X, L^{n}\right),
$$

the homogeneous coordinate ring associated to $L$. In particular, we would like criteria for the properties:

(a) $R$ is generated by

$$
R_{1}=\Gamma(X, L)
$$

(b) If $S^{*} R_{1}$ is the symmetric algebra on $R_{1}$ over $k$, and $I$ is the kernel:

$$
0 \rightarrow I \rightarrow S^{*} R_{1} \rightarrow R
$$

then $I$ is generated, as $S^{*} R_{1}$-module, by the quadratic relations $I_{2}$.

In the first place, there are very general methods, based on CASTELNUOVo's lemma, that give results of this type. I can prove:

Proposition 1. If $L$ is replaced by $L^{n}$, where $n \geqq \operatorname{dim} X+2$, then both (a) and (b) are true.

This is not very useful, however, for the present theory. In the theorems that we want, we shall assume much less about the sheaf. However, as a consequence of our somewhat peculiar methods, we only obtain criteria for:

(a) $: R_{\left(2^{n}\right)}$ generated by the $\left(2^{n}\right)$-th symmetric power of $R_{1}$, for all $n$.

(b) $I_{\left(2^{n}\right)}$ generated by $I_{2}$ times $S^{2^{n-2}}\left(R_{1}\right)$ for all $n$.

Although these are strange conditions, still if they are true in some case, then the following also hold:

(a) ${ }^{\prime \prime}: R_{m}$ is generated by the $m$-th symmetric power of $R_{1}$, for all $m$ sufficiently large.

(b) ${ }^{\prime \prime}: I_{m}$ is generated by $I_{2}$ times $S^{m-2}\left(R_{1}\right)$ for all $m$ sufficiently large. Incidentally, (a)" is equivalent to the condition that $L$ be very ample.

Lemma. (a)' $\Rightarrow(a)^{\prime \prime}$,

$$
\text { (b) }{ }^{\prime} \Rightarrow(\mathrm{b})^{\prime \prime} \text {. }
$$

Proof. Assume (a)'. For all integers $m$, let

$$
R(m)=\bigoplus_{k=0}^{\infty} R_{m k}
$$


It is well known that for all large $m, R(m)$ is generated by $R(m)_{1}=R_{m}$. In particular, for large $n, R\left(2^{n}\right)$ is generated by $R_{\left(2^{n}\right)}$ and hence by $S^{*} R_{1}$. On the other hand, $R$ is an $R\left(2^{n}\right)$-module of finite type for all $n$. Therefore, a fortiori, $R$ is an $S^{*} R_{1}$-module of finite type. Now let $M=R / \operatorname{Im} S^{*} R_{1}$. Consider $M$ as an $S^{*} R_{1}$-module. It is of finite type since $R$ is; and $M_{\left(2^{n}\right)}=(0)$ for all $n$. But, given any graded $R$-module $N$ of finite type, either $N_{n} \neq(0)$ for all large $n$, or $N_{n}=(0)$ for all large $n$. Hence (a) $)^{\prime \prime}$ holds.

Assume (b)'. Let $J \subset I$ be the ideal generated by $I_{2}$. Then $I / J$ is an $S^{*} R_{1}$-module of finite type such that $(I / J)_{\left(2^{n}\right)}=(0)$ for all $n$. As above, this implies that $(I / J)_{m}=(0)$ for all large $m$, hence (b)" holds. Q.E.D.

Conditions (a)' and (b)' are exactly what we can get hold of by the theory of $\S 3$. To set this up, first choose for every integer $n$ a symmetric $\vartheta$-structure on $L^{2 n}$ :

$$
f_{n}: \mathscr{G}\left(L^{2^{n}}\right) \stackrel{\sim}{\longrightarrow} \mathscr{G}\left(2^{n} \delta\right),
$$

such that $\left(f_{n}, f_{n+1}\right)$ is always a symmetric $\vartheta$-structure for $\left(L^{2 n}, L^{2 n+1}\right)$. This is possible by Proposition 7 of $\S 2$. This induces isomorphisms

$$
\varphi_{n}: \Gamma\left(X, L^{2^{n}}\right) \stackrel{\sim}{\longrightarrow} V\left(2^{n} \delta\right)=\left\{\begin{array}{c}
\text { vector space of } \\
\text { functions on } \\
K\left(2^{n} \delta\right)
\end{array}\right\}
$$

for all $n$, unique up to scalars. Moreover, for all $n$, let the function $q_{n}(x)$ on $K\left(2^{n} \delta\right)$ correspond to the linear functional on $\Gamma\left(X, L^{2 n}\right)$ defined by evaluating sections at 0 . Using only the functions $q_{n}$, we can define the following ring:

$R^{*}$ : graded polynomial ring generated by $\oplus_{n=0}^{\infty} V\left(2^{n} \delta\right)$, where elements of $V\left(2^{n} \delta\right)$ are assigned degree $2^{n}$, modulo the relations

$$
f \cdot f^{\prime}-g
$$

for all $\left\{\begin{aligned} f, f^{\prime} \in V\left(2^{n} \delta\right) \\ \quad g \in V\left(2^{n+1} \delta\right), \text { some } n \text { such that }\end{aligned}\right.$

$$
g(x)=\sum_{y \in x+K\left(2^{n} \delta\right)} f(x+y) \cdot f^{\prime}(x-y) \cdot q_{n+1}(y) .
$$

[Here, as in $\S 3$, we identify the groups $K\left(2^{n} \delta\right)$ as subgroups of each other:

It follows that

$$
\left.K(\delta) \subset K(2 \delta) \subset \cdots \subset K\left(2^{n} \delta\right) \subset \cdots .\right]
$$

$$
R_{2^{n}}^{*}=V\left(2^{n} \delta\right)
$$

23* 
and if any integer $N$ is written as a sum

$$
N=2^{n_{1}}+2^{n_{2}}+\cdots+2^{n_{k}}
$$

with $n_{1}, n_{2}, \ldots, n_{k}$ distinct, then

$$
R_{N}^{*} \cong V\left(2^{n_{1}} \delta\right) \otimes \cdots \otimes V\left(2^{n_{k}} \delta\right) /(\text { something) } .
$$

In any case, there is a canonical map

$$
\Phi: \quad R^{*} \rightarrow R
$$

defined by extending the maps $\varphi_{n}$ to a homomorphism of all of $R^{*}$.

Lemma. i) $R^{*}$ is a finitely generated k-algebra and the kernel of $\Phi$ is finite-dimensional,

ii) $\Phi_{N}: R_{N}^{*} \rightarrow R_{N}$ is an isomorphism if $N$ is a power of 2 ,

iii) if $L$ is very ample, then $\Phi_{N}$ is an isomorphism for all but a finite number of $N$.

Proof. First of all, (ii) is obvious. Since $L$ is ample, $R_{2^{n}}$ is spanned by $R_{n} \otimes R_{n}$ for all sufficiently large $n$. Therefore, $R_{2^{n+1}}^{*}$ is spanned by $R_{2^{n}}^{*} \otimes R_{2^{n}}^{*}$ for all sufficiently large $n$. Therefore $R^{*}$ is finitely generated. If $J=\operatorname{ker}(\Phi)$, then $J$ is a finitely generated $R^{*}$-module; since $J$ is zero in degrees $2^{n}$, it follows that $J$ is finite-dimensional. As for (iii), if $L$ is very ample, $R_{1}$ generates all but a finite-dimensional piece of $R$. Therefore the subring of $R^{*}$ generated by $R_{1}^{*}$ already goes onto all but a finite-dimensional piece of $R$. Q.E.D.

This lemma means that if $L$ is very ample, the abstract ring $R^{*}$ defined using only the family of null-value functions $q_{n}$, is essentially isomorphic to $R$. We can therefore calculate in $R^{*}$ to obtain results about the ring $R$. The first result of this type is:

Theorem 1. If $H(L)$ contains $X_{4}$, the group of points of order 4 , then $\Gamma\left(X, L^{2}\right)$ is spanned by $S^{2}\{\Gamma(X, L)\}$.

Proof. We will show that every function on $K(2 \delta)$ is a linear combination of functions $f * f^{\prime}$, where $f, f^{\prime}$ are functions on $K(\delta)$. (Notation as in $\S 3)$. It will be convenient to work with the delta functions on $K(\delta), K(2 \delta)$ : we write $\delta_{a}$ for the function:

$$
\begin{array}{rlll}
\delta_{a}\left(a^{\prime}\right)=1 & \text { if } & a^{\prime}=a \\
=0 & \text { if } & a^{\prime} \neq a .
\end{array}
$$

There should be no cause for confusion with the use of $\delta$ in "level $\delta$ ". We let $Z_{2}$ denote the subgroup of $K(\delta)$ of points of order 2 . 
First, we calculate $\delta_{a+b} * \delta_{a-b}$, where $a, b \in K(2 \delta)$ and $a+b \in K(\delta)$

$$
\left(\delta_{a+b} * \delta_{a-b}\right)(x)=\sum_{z \in x+K(\delta)} \delta_{a+b}(x+z) \cdot \delta_{a-b}(x-z) \cdot q_{1}(z) .
$$

The term inside is 0 unless

$$
\begin{aligned}
& x+z=a+b \\
& x-z=a-b .
\end{aligned}
$$

These imply that $x \in a+Z_{2}$. Conversely, if $x \in a+Z_{2}$, then the sum has exactly one non-zero term, viz., $q_{1}(a+b-x)$. Therefore:

$$
\begin{aligned}
\delta_{a+b} * \delta_{a-b} & =\sum_{x \in a+\mathrm{Z}_{2}} q_{1}(a+b-x) \cdot \delta_{x} \\
& =\sum_{\eta \in Z_{2}} q_{1}(b+\eta) \cdot \delta_{a+\eta} .
\end{aligned}
$$

These functions certainly span the image of $S^{2}[V(\delta)]$. However, it is convenient to use a slightly different basis. Let $l$ be a homomorphism from $Z_{2}$ to $\{ \pm 1\}$. Then

$$
\begin{aligned}
\sum_{\eta \in Z_{2}} l(\eta) \delta_{a+b+\eta} * \delta_{a-b+\eta} & =\sum_{\eta_{1}, \eta_{2} \in Z_{2}} l\left(\eta_{1}\right) \cdot q_{1}\left(a+b+\eta_{1}-\left(a+\eta_{2}\right)\right) \cdot \delta_{a+\eta_{2}} \\
& =\left[\sum_{\eta \in Z_{2}} l(\eta) \cdot q_{1}(b+\eta)\right] \cdot\left[\sum_{\eta \in Z_{2}} l(\eta) \cdot \delta_{a+\eta}\right] .
\end{aligned}
$$

If the image of $S^{2}[V(\delta)]$ contains all the functions $\sum l(\eta) \delta_{a+\eta}$, then it contains everything. Therefore, the Theorem is true if we can prove:

$(*)$

$$
\left\{\begin{array}{l}
\text { For every } a \in K(2 \delta) \text { and every homomorphism } l: Z_{2} \rightarrow\{ \pm 1\} \\
\text { there is an element } b \in a+K(\delta) \text { such that }
\end{array}\right.
$$

To prove this, set

$$
\sum_{\eta \in Z_{2}} l(\eta) \cdot q_{1}(b+\eta) \neq 0 .
$$

and

$$
F=\sum_{\eta \in Z_{2}} \delta_{2 a+\eta}
$$

Now calculate $F * G$ :

$$
G=\sum_{\eta \in Z_{2}} l(\eta) \delta_{\eta}
$$

$$
(F * G)(x)=\sum_{z \in x+K(\delta)} F(x+z) \cdot G(x-z) \cdot q_{1}(z) .
$$

The inside term is zero unless:

$$
\begin{aligned}
& x+z=2 a+\eta_{1} \\
& x-z=\eta_{2}, \quad \eta_{1}, \eta_{2} \in Z_{2} .
\end{aligned}
$$


These imply $4 x=4 a$. Let $Z_{4}$ be the group of elements of $K(2 \delta)$ of order 4 . By our hypothesis on $L, Z_{4}$ is a subgroup of $K(\delta)$. Now suppose $x \in a+Z_{4}$. Then the sum works out to be:

Therefore:

$$
\begin{aligned}
& =\sum_{\eta_{2} \in Z_{2}} F\left(2 x+\eta_{2}\right) \cdot G\left(\eta_{2}\right) \cdot q_{1}\left(x+\eta_{2}\right) \\
& =\sum_{\eta \in Z_{2}} l(\eta) \cdot q_{1}(x+\eta) .
\end{aligned}
$$

$$
F * G=\sum_{x \in a+Z_{4}}\left(\sum_{\eta \in Z_{2}} l(\eta) \cdot q_{1}(x+\eta)\right) \cdot \delta_{x} .
$$

Now assume (*) is false. Since $Z_{4} \subset K(\delta)$, it follows that all the coefficients $\sum l(\eta) q_{1}(x+\eta)$ are zero. Therefore $F * G=0$. But $F \neq 0$ and $G \neq 0$, and the homogeneous coordinate ring of an abelian variety is well known to be an integral domain! This contradiction shows that (*) must hold. Q.E.D.

Corollary. If $H(L) \supset X_{4}$, then $R^{*}$ is generated by $R_{1}^{*}$ and (a)' and (a)" are true for the homogeneous coordinate ring

$$
\bigoplus_{n=0}^{\infty} \Gamma\left(X, L^{n}\right)
$$

In particular, $L$ is very ample.

The inequality proven about $q_{1}$ in the course of this proof will be quite useful in the sequel. To bring out the general picture I want to state the analogous fact about the values of $q$ when expanded in characters of an arbitrary subgroup $Z$ :

As above, let

$$
Z_{2} \subset Z \subset 2 K(2 \delta) \text {. }
$$

$$
\vartheta(a, l)=\sum_{\eta \in Z} l(\eta) \cdot q_{1}(a+\eta), \quad \text { if } \quad l \in \hat{Z}, \quad a \in K(2 \delta) .
$$

The result is:

a) for all $a \in K(2 \delta), l \in \hat{Z}$, there exists an element $\zeta \in \frac{1}{2} Z$ such that

$$
\vartheta(a+\zeta, l) \neq 0,
$$

b) for all $a \in K(2 \delta), l \in \hat{Z}$, there exists an element $m \in(\hat{Z})_{2}$, i.e., a character of $Z / 2 Z$, such that

$$
\vartheta(a, l+m) \neq 0 \text {. }
$$

These facts are proven by an obvious generalization of the argument used to prove $(*)$. 
The second result which we get by calculating in $R^{*}$ is this:

Theorem 2. Let

$$
\begin{aligned}
& I_{2}=\operatorname{Ker}\left\{S^{2} R_{1} \rightarrow R_{2}\right\} \\
& I_{4}=\operatorname{Ker}\left\{S^{4} R_{1} \rightarrow R_{4}\right\} .
\end{aligned}
$$

Assume $H(L)$ contains $X_{4}$. Then $I_{4}$ is generated by $R_{2} \otimes I_{2}$.

Proof. First of all, note that by Theorem $1, R_{2}$ and $R_{4}$ are quotients of $S^{2} R_{1}$ and $S^{4} R_{1}$, respectively. To compute $R_{2} \otimes I_{2}$, we use the obvious remark:

Lemma. Let $V$ be a $k$-vector-space, and let $I \subset S^{2} V$ be any subspace. For all elements $a, b \in V$, let

Then

$$
x_{a, b}=\text { image of } a \cdot b \text { in } S^{2} V / I .
$$

$$
S^{4} V / S^{2} V \cdot I \cong S^{2}\left[S^{2} V / I\right] /\left\{\begin{array}{c}
\text { span of the elements } \\
x_{a, b} \cdot x_{c, d}-x_{a, c} \cdot x_{b, d}
\end{array}\right\} \text {. }
$$

For all $a, b \in R_{1}$, let $x_{a, b}$ denote the product of $a$ and $b$ in $R_{2}$. Then the lemma tells us that

$$
S^{4} R_{1} / R_{2} \cdot I_{2}=S^{2} R_{2} /\left\{\begin{array}{l}
\text { span of the elements } \\
x_{a, b} \cdot x_{c, d}-x_{a, c} \cdot x_{b, d}
\end{array}\right\} .
$$

Therefore it suffices to prove that the canonical map:

is injective.

$$
S^{2} R_{2} /\left\{\begin{array}{l}
\text { span of the elements } \\
x_{a, b} \cdot x_{c, d}-x_{a, c} \cdot x_{b, d}
\end{array}\right\} \rightarrow R_{4}
$$

In order to do this, the key point is to compute in terms of the most carefully chosen bases of $R_{1}, R_{2}$ and $R_{4}$.

(I) In $R_{1}$, we use the functions $\delta_{a}, a \in K(\delta)$.

(II) In $R_{2}$, we use the functions

$$
Y_{a, l}=\sum_{\eta \in Z_{2}} l(\eta) \cdot \delta_{a+\eta}
$$

where $Z_{2}=$ subgroup of $K(\delta)$ of points of order 2 and $l \in \hat{Z}_{2}$.

(III) In $R_{4}$, we use the functions

$$
Z_{a, l}=\sum_{\eta \in Z_{4}} l(\eta) \cdot \delta_{a+\eta}
$$

where $Z_{4}=$ subgroup of $K(\delta)$ of points of order 4 and $l \in \hat{Z}_{4}$. 
Moreover, set

$$
\begin{aligned}
& q_{1}(a, l)=\sum_{\eta \in Z_{2}} l(\eta) \cdot q_{1}(a+\eta), \quad l \in \hat{Z}_{2}, \quad a \in K(2 \delta) \\
& q_{2}(a, l)=\sum_{\eta \in Z_{4}} l(\eta) \cdot q_{2}(a+\eta), \quad l \in \hat{Z}_{4}, \quad a \in K(4 \delta) .
\end{aligned}
$$

Now if $a, b \in K(2 \delta)$ and $a+b \in K(\delta)$, calculate

$$
\begin{aligned}
\sum_{l \in \hat{Z}_{2}} q_{1}(b, l) \cdot Y_{a, l} & =\sum_{\substack{l \in \hat{Z}_{2} \\
\eta, \eta^{\prime} \in Z_{2}}} l(\eta) \cdot l\left(\eta^{\prime}\right) \cdot q_{1}(b+\eta) \cdot \delta_{a+\eta^{\prime}} \\
& =2^{g} \sum_{\eta \in Z_{2}} q_{1}(b+\eta) \cdot \delta_{a+\eta} \\
& =2^{g} \delta_{a+b} * \delta_{a-b}
\end{aligned}
$$

(cf. proof of Theorem 1). In $S^{2} R_{2}$, denote the symmetric product by $\odot$. Then the problem is to show that:

$$
S^{2} R_{2} /\left\{\begin{array}{c}
\text { span of the elements: } \\
\sum_{l} q_{1}(b, l) \cdot Y_{a, l} \odot \sum_{l} q_{1}(d, l) \cdot Y_{c, l}- \\
-\sum_{l} q_{1}(a+e, l) \cdot Y_{-b-e, l} \odot \sum_{l} q_{1}(c+e, l) \cdot Y_{-d-e, l} \\
\text { for all } a, b, c, d, e \in K(2 \delta) \text { such that } \\
a+b+c+d=-2 e \\
a+b, c+d \in K(\delta)
\end{array}\right\}
$$

is isomorphic to $R_{4}$. Let $L$ be the span of the elements inside the braces. Then take a typical generator of $L$, and write

$$
\begin{aligned}
a & =A+B \\
c & =A-B \\
-b-e & =A+C \\
-d-e & =A-C
\end{aligned}
$$

with $A, B, C \in K(4 \delta)$. It follows that $L$ is the span of the elements

$$
\begin{aligned}
\sum_{l, l^{\prime} \in \hat{Z}_{2}} q_{1}(A+C+e, l) \cdot q_{1}\left(A-C+e, l^{\prime}\right) \cdot Y_{A+B, l} \odot Y_{A-B, l^{\prime}}- \\
-\sum_{l, l^{\prime} \in \hat{\mathrm{Z}}_{2}} q_{1}(A+B+e, l) \cdot q_{1}\left(A-B+e, l^{\prime}\right) \cdot Y_{A+C, l} \odot Y_{A-C, l^{\prime}}
\end{aligned}
$$

for all $A, B, C, e \in K(4 \delta)$ such that

and

$$
A+B, A+C, e \in K(2 \delta)
$$

$$
B-C+e \in K(\delta)
$$


Next, we replace these by another set of generators of $L$ obtained by a partial Fourier transformation: in a typical generator, replace

$$
\begin{array}{lll}
A & \text { by } & A+\zeta \\
B & \text { by } & B+\zeta^{\prime} \\
C & \text { by } & C+\zeta^{\prime \prime} \\
e & \text { by } & e-\zeta
\end{array}
$$

where $\zeta, \zeta^{\prime}, \zeta^{\prime \prime} \in Z_{4}$. This is $0 K$, since $Z_{4} \subset K(\delta)$. Let $m, m^{\prime}, m^{\prime \prime}$ be elements of $\hat{Z}_{4}$. Multiply by $m(\zeta) \cdot m^{\prime}\left(\zeta^{\prime}\right) \cdot m^{\prime \prime}\left(\zeta^{\prime \prime}\right)$ and sum over $\zeta, \zeta^{\prime}, \zeta^{\prime \prime}$. This gives us generators for $L$ of the form:

$$
\begin{aligned}
\sum_{l, l^{\prime} \in \hat{Z}_{2}}\left[\sum_{\zeta^{\prime \prime} \in Z_{4}} m^{\prime \prime}\left(\zeta^{\prime \prime}\right) \cdot q_{1}\left(A+C+e+\zeta^{\prime \prime}, l\right) \cdot q_{1}\left(A-C+e-\zeta^{\prime \prime}, l^{\prime}\right)\right] \times \\
\times\left[\sum_{\zeta, \zeta^{\prime} \in Z_{4}} m(\zeta) \cdot m^{\prime}\left(\zeta^{\prime}\right) \cdot Y_{A+B+\zeta+\zeta^{\prime}, l} \odot Y_{A-B+\zeta-\zeta^{\prime}, l^{\prime}}\right]- \\
-\sum_{l, l^{\prime} \in \hat{Z}_{2}}\left[\sum_{\zeta^{\prime} \in Z_{4}} m^{\prime}\left(\zeta^{\prime}\right) \cdot q_{1}\left(A+B+e+\zeta^{\prime}, l\right) \cdot q_{1}\left(A-B+e-\zeta^{\prime}, l^{\prime}\right)\right] \times \\
\times\left[\sum_{\zeta, \zeta^{\prime \prime} \in Z_{4}} m(\zeta) \cdot m^{\prime \prime}\left(\zeta^{\prime \prime}\right) \cdot Y_{A+C+\zeta+\zeta^{\prime \prime}, l} \odot Y_{A-C+\zeta-\zeta^{\prime \prime}, l^{\prime}}\right] .
\end{aligned}
$$

This expression simplifies a great deal. The terms in the 1 st sum over $l$ and $l^{\prime}$ are 0 unless $l$ and $l^{\prime}$ satisfy:

$$
\begin{aligned}
& l(2 x)=\left(m+m^{\prime}\right)(x), \text { all } x \in Z_{4} \\
& l^{\prime}(2 x)=\left(m-m^{\prime}\right)(x), \text { all } x \in Z_{4} \\
& m^{\prime \prime}(x)=\left(l+l^{\prime}\right)(x), \quad \text { all } x \in Z_{2} \text {. }
\end{aligned}
$$

In particular, the whole 1 st term is 0 unless $m, m^{\prime}$ and $m^{\prime \prime}$ are equal on $Z_{2}$. Similarly, in the 2 nd term, we get 0 unless

$$
\begin{aligned}
l(2 x) & =\left(m+m^{\prime \prime}\right)(x), & \text { all } & x \in Z_{4} \\
l^{\prime}(2 x) & =\left(m-m^{\prime \prime}\right)(x), & \text { all } & x \in Z_{4} \\
m^{\prime}(x) & =\left(l+l^{\prime}\right)(x), & \text { all } & x \in Z_{2} .
\end{aligned}
$$

Therefore the whole expression is 0 unless $m, m^{\prime}$ and $m^{\prime \prime}$ are equal on $Z_{2}$. We may as well set

$$
\begin{aligned}
& m=k+k^{\prime} \\
& m^{\prime}=k-k^{\prime} \\
& m^{\prime \prime}=k-k^{\prime}+2 k^{*}
\end{aligned}
$$


for suitable elements $k, k^{\prime}, k^{*} \in \hat{Z}_{4}$. Then, up to a constant, the expression reduces to:

$$
\begin{aligned}
& {\left[\sum_{\zeta \in Z_{4}}\left(k-k^{\prime}+2 k^{*}\right)(\zeta) \cdot q_{1}(A+C+e+\zeta, k) \cdot q_{1}\left(A-C+e-\zeta, k^{\prime}\right)\right] \times} \\
& \times\left[\sum_{\zeta \in Z_{4}}\left(k+k^{\prime}\right)(\zeta) \cdot Y_{A+B+\zeta, k} \odot Y_{A-B+\zeta, k^{\prime}}\right]- \\
& -\left[\sum_{\zeta \in Z_{4}}\left(k-k^{\prime}\right)(\zeta) \cdot q_{1}\left(A+B+e+\zeta, k+k^{*}\right) \cdot q_{1}\left(A-B+e-\zeta, k^{\prime}-k^{*}\right)\right] \times \\
& \quad \times\left[\sum_{\zeta \in Z_{4}}\left(k+k^{\prime}\right)(\zeta) \cdot Y_{A+C+\zeta, k+k^{*}} \odot Y_{A-C+\zeta, k^{\prime}-k^{*}}\right] .
\end{aligned}
$$

The 1st and the 3rd bracketed expressions can be written in terms of $q_{2}$ : let $a, b \in K(4 \delta)$ and let $l, l \in \hat{Z}_{4}$ such that $a+b \in K(2 \delta)$. Then

$$
\begin{aligned}
\sum_{\zeta \in Z_{4}} & \left(l+l^{\prime}\right)(\zeta) \cdot q_{1}(a+b+\zeta, l) \cdot q_{1}\left(a-b-\zeta, l^{\prime}\right) \\
& =\sum_{\substack{\zeta \in Z_{4} \\
\eta, \eta^{\prime} \in Z_{2}}} l(\zeta+\eta) \cdot l^{\prime}\left(\zeta+\eta^{\prime}\right) \cdot q_{1}(a+b+\zeta+\eta) \cdot q_{1}\left(a-b-\zeta-\eta^{\prime}\right) \\
& =\sum_{\zeta_{1}, \zeta_{2} \in Z_{4}} l\left(\zeta_{1}+\zeta_{2}\right) \cdot l^{\prime}\left(\zeta_{1}-\zeta_{2}\right) \cdot q_{1}\left(a+b+\zeta_{1}+\zeta_{2}\right) \cdot q_{1}\left(a-b+\zeta_{2}-\zeta_{1}\right) \\
& =\sum_{\zeta_{1}, \zeta_{2} \in Z_{4}} l\left(\zeta_{1}+\zeta_{2}\right) \cdot l^{\prime}\left(\zeta_{1}-\zeta_{2}\right) \cdot q_{2}\left(a+\zeta_{2}\right) \cdot q_{2}\left(b+\zeta_{1}\right)
\end{aligned}
$$

(cf. §3)

$$
=q_{2}\left(a, l-l^{\prime}\right) \cdot q_{2}\left(b, l+l^{\prime}\right) \text {. }
$$

Substituting this formula, we find that $L$ is spanned by the elements

$$
\begin{aligned}
& q_{2}\left(A+e, k+k^{\prime}+2 k^{*}\right) \times \\
& \quad \times\left\{\begin{array}{l}
q_{2}\left(C, k-k^{\prime}+2 k^{*}\right) \cdot \sum_{\zeta \in Z_{4}}\left(k+k^{\prime}\right)(\zeta) \cdot Y_{A+B+\zeta, k} \odot Y_{A-B+\zeta, k^{\prime}}- \\
-q_{2}\left(B, k-k^{\prime}\right) \cdot \sum_{\zeta \in Z_{4}}\left(k+k^{\prime}\right)(\zeta) \cdot Y_{A+C+\zeta, k+k^{*}} \odot Y_{A-C+\zeta, k^{\prime}-k^{*}}
\end{array}\right\} .
\end{aligned}
$$

What happens to these sums when they are pushed from $S^{2} R_{2}$ into $R_{4}$ ? We calculate

$$
\begin{aligned}
\sum_{\zeta \in Z_{4}}\left(k+k^{\prime}\right)(\zeta) \cdot Y_{A+B+\zeta, k} * Y_{A-B+\zeta, k^{\prime}} \\
=\sum_{\substack{\zeta \in Z_{4} \\
\eta, \eta^{\prime} \in Z_{2}}} k(\zeta+\eta) \cdot k^{\prime}\left(\zeta+\eta^{\prime}\right) \cdot \delta_{A+B+\zeta+\eta} * \delta_{A-B+\zeta+\eta^{\prime}} \\
=\sum_{\zeta_{1}, \zeta_{2} \in Z_{4}} k\left(\zeta_{1}+\zeta_{2}\right) \cdot k^{\prime}\left(\zeta_{1}-\zeta_{2}\right) \cdot \delta_{A+B+\zeta_{1}+\zeta_{2}} * \delta_{A-B+\zeta_{1}-\zeta_{2}} \\
=\sum_{\zeta_{1}, \zeta_{2} \in Z_{4}} k\left(\zeta_{1}+\zeta_{2}\right) \cdot k^{\prime}\left(\zeta_{1}-\zeta_{2}\right) \cdot q_{2}\left(B+\zeta_{2}\right) \cdot \delta_{A+\zeta_{1}}
\end{aligned}
$$


(cf. proof of Theorem 1)

$$
=q_{2}\left(B, k-k^{\prime}\right) \cdot Z_{A, k+k^{\prime}} .
$$

Therefore, in order to check that $L$ is big enough, it is necessary and sufficient, for every pair of terms

$$
T_{i}=\sum_{\zeta \in Z_{4}}\left(k_{i}+k_{i}^{\prime}\right)(\zeta) \cdot Y_{A_{i}+B_{i}+\zeta, k_{i}} \odot Y_{A_{i}-B_{i}+\zeta, k_{i}^{\prime}}, \quad i=1,2
$$

[here $\left.A_{i}, B_{i} \in K(4 \delta), A_{i}+B_{i} \in K(2 \delta), k_{i}, k_{i}^{\prime} \in \hat{Z}_{4}\right]$ for which $A_{1}=A_{2}$ and $k_{1}+k_{1}^{\prime}=k_{2}+k_{2}^{\prime}$, to show that $c_{1} T_{1}+c_{2} T_{2}$ is in $L$ for some non-zero pair $\left(c_{1}, c_{2}\right)$ of constants. [One must check at this point that $S^{2} R_{2}$ is itself spanned by these terms, but this is nearly obvious.]

Now set

$$
\begin{aligned}
\Sigma(A, & \left.B, C ; k, k^{\prime}, k^{*}\right) \\
= & q_{2}\left(C, k-k^{\prime}+2 k^{*}\right) \cdot \sum_{\zeta \in Z_{4}}\left(k+k^{\prime}\right)(\zeta) \cdot Y_{A+B+\zeta, k} \odot Y_{A-B+\zeta, k^{\prime}}- \\
& -q_{2}\left(B, k-k^{\prime}\right) \cdot \sum_{\zeta \in Z_{4}}\left(k+k^{\prime}\right)(\zeta) \cdot Y_{A+C+\zeta, k+k^{*}} \odot Y_{A-C+\zeta, k^{\prime}-k^{*}} .
\end{aligned}
$$

I claim it is enough to show that for every $A, B, C \in K(4 \delta), k, k^{\prime}, k^{*} \in \hat{Z}_{4}$ such that $A+B, A+C \in K(2 \delta)$, the expression $\Sigma\left(A, B, C ; k, k^{\prime}, k^{*}\right)$ is in $L$. Namely, if $T_{1}$ and $T_{2}$ are any pair of terms as above, then

$$
\Sigma\left(A_{1}, B_{1}, B_{2} ; k_{1}, k_{1}^{\prime}, k_{2}-k_{1}\right)
$$

is clearly of the form $c_{1} T_{1}+c_{2} T_{2}$. The only problem is that $c_{1}$ and $c_{2}$ might be 0. But $T_{1}$ is mapped into $c_{2} Z_{A_{1}, k_{1}+k_{1}^{\prime}}$ in $R_{4}$ and $T_{2}$ is mapped into $c_{1} Z_{A_{1}, k_{1}+k_{1}^{\prime}}$ in $R_{4}$. Therefore $c_{1}=c_{2}=0$ only if $T_{1}$ and $T_{2}$ go to 0 in $R_{4}$. But since $S^{2} R_{2}$ is mapped onto $R_{4}$ by Theorem 1 , for every term $T_{1}$, there is some term $T_{3}$ with $A_{3}=A_{1}, k_{3}+k_{3}^{\prime}=k_{1}+k_{1}^{\prime}$ such that $T_{3}$ is mapped to a non-zero multiple of $Z_{A_{1}, k_{1}+k_{1}}$. Suppose all the $\Sigma$ 's are in $L$. Then

$$
\begin{aligned}
& \Sigma\left(A_{1}, B_{1}, B_{3} ; k_{1}, k_{1}^{\prime}, k_{3}-k_{1}\right) \in L \\
& \Sigma\left(A_{2}, B_{2}, B_{3} ; k_{2}, k_{2}^{\prime}, k_{3}-k_{2}\right) \in L .
\end{aligned}
$$

In other words, $L$ contains expressions of the form $d_{1} T_{1}+d_{3}^{\prime} T_{3}$, with $d_{1} \neq 0$; and $d_{2} T_{2}+d_{3}^{\prime \prime} T_{3}$ with $d_{2} \neq 0$. Therefore $L$ contains an expression $c_{1} T_{1}+c_{2} T_{2}$ with $c_{1}$ or $c_{2} \neq 0$.

The identity:

$$
\begin{aligned}
q_{2}\left(C, k-k^{\prime}+2 k^{*}\right) \cdot \Sigma & \left(A, B, D ; k, k^{\prime}, l^{*}\right)+ \\
& +q_{2}\left(B, k-k^{\prime}\right) \cdot \Sigma\left(A, D, C ; k+l^{*}, k^{\prime}-l^{*}, k^{*}-l^{*}\right) \\
= & q_{2}\left(D, k-k^{\prime}+2 l^{*}\right) \cdot \Sigma\left(A, B, C ; k, k^{\prime}, k^{*}\right)
\end{aligned}
$$


is easy to check. [Here $A, B, C, D$ are in $K(4 \delta), k, k^{\prime}, k^{*}, l^{*} \in \hat{Z}_{4}$ and $A+B, A+C, A+D \in K(2 \delta)$.] We have proven, that for all $A, B, C \in K(4 \delta)$ and $k, k^{\prime}, k^{*} \in \hat{Z}_{4}$ such that $A+B, A+C \in K(2 \delta)$, then

$$
q_{2}\left(A+e, k+k^{\prime}+2 k^{*}\right) \cdot \Sigma\left(A, B, C ; k, k^{\prime}, k^{*}\right) \in L
$$

for any element $e \in K(4 \delta)$ such that

$$
e \in B-C+K(\delta)
$$

This embarrassing factor $q_{2}\left(A+e, k+k^{\prime}+2 k^{*}\right)$ is the crux of the problem at this point. The $e$ here gives us very little flexibility: if it is varied by an element of $Z_{4}$ (which is permitted), the $q_{2}$ is multiplied only by a root of 1 . So if one of these vanishes, all of them do. And since we have only assumed that $Z_{4} \subset K(\delta)$, this is really the only variation possible in $e$. We may as well set $e=B-C$ therefore; this gives us the fact:

$$
q_{2}\left(A+B-C, k+k^{\prime}+2 k^{*}\right) \cdot \Sigma\left(A, B, C ; k, k^{\prime}, k^{*}\right) \in L .
$$

But now the cocycle identity gives us also the fact:

$$
\begin{aligned}
& q_{2}\left(A+B-D, k+k^{\prime}+2 l^{*}\right) \cdot q_{2}\left(A+D-C, k+k^{\prime}+2 k^{*}-2 l^{*}\right) \times \\
& \times q_{2}\left(D, k-k^{\prime}+2 l^{*}\right) \cdot \Sigma\left(A, B, C ; k, k^{\prime}, k^{*}\right) \\
= & q_{2}\left(C, k-k^{\prime}+2 k^{*}\right) \cdot q_{2}\left(A+D-C, k+k^{\prime}+2 k^{*}-2 l^{*}\right) \times \\
& \times\left[q_{2}\left(A+B-D, k+k^{\prime}+2 l^{*}\right) \cdot \Sigma\left(A, B, D ; k, k^{\prime}, l^{*}\right)\right]+ \\
& +q_{2}\left(B, k-k^{\prime}\right) \cdot q_{2}\left(A+B-D, k+k^{\prime}+2 l^{*}\right) \times \\
& \times\left[q_{2}\left(A+D-C, k+k^{\prime}+2 k^{*}-2 l^{*}\right) \cdot \Sigma\left(A, D, C, k+l^{*}, k^{\prime}-l^{*}, k^{*}-l^{*}\right)\right] \\
\in & L,
\end{aligned}
$$

for all $l^{*} \in \hat{Z}_{4}, D \in A+K(2 \delta)$. The product of $3 q_{2}$ 's at the beginning of this equation can be made non-zero by an appropriate choice of $l^{*}$ and $D$ ! This is the content of:

Triple Vanishing Lemma. Let $A_{1}, A_{2}, A_{3}$ be any three elements of $K(4 \delta)$; let $k_{1}, k_{2}, k_{3}$ be any three elements of $\hat{Z}_{4}$. Then there exists

such that

$$
\begin{aligned}
& \zeta \in \mathrm{Z}_{\mathbf{8}} \quad(=\text { points of order } 8 \text { in } K(2 \delta)) \\
& l \in 2 \hat{Z}_{4}
\end{aligned}
$$

$$
\prod_{i=1}^{3} q_{2}\left(A_{i}+\zeta, k_{i}+l\right) \neq 0 .
$$


Proof. To show this, we first establish the following general formula:

Lemma. Let $L$ be a totally symmetric ample invertible sheaf on $X$ of separable type $\delta$. Choose compatible $\vartheta$-structures for $L, L^{2}, L^{4}$. Let $A_{1}, A_{2}, A_{3}, A_{4} \in K(\delta)$, and let $q_{1} \in V(\delta)$ correspond to evaluation at 0 . Then

$\sum_{\eta \in Z_{2}} \delta_{A_{1}+\eta} * \delta_{A_{2}+\eta} * \delta_{A_{3}+\eta} * \delta_{A_{4}+\eta}$

$=\sum_{\substack{E \in K(4 \delta) \\ 4 E=A_{1}+A_{2}+A_{3}+A_{4}}} q_{1}\left(A_{1}+A_{2}-2 E\right) \cdot q_{1}\left(A_{1}+A_{3}-2 E\right) \cdot q_{1}\left(A_{1}+A_{4}-2 E\right) \cdot \delta_{E}$.

Proof. Write

$$
\begin{aligned}
& A_{1}=\alpha+\delta+\beta \\
& A_{2}=\alpha+\delta-\beta \\
& A_{3}=-\alpha+\delta+\gamma \\
& A_{4}=-\alpha+\delta-\gamma
\end{aligned}
$$

where $\beta, \gamma \in K(2 \delta), \alpha, \delta \in K(4 \delta)$. Then combining the 1 st 2 and last 2 terms by the formula in Theorem 1, we find

$$
\begin{aligned}
\sum_{\eta \in Z_{2}} \delta_{A_{1}+\eta} & * \delta_{A_{2}+\eta} * \delta_{A_{3}+\eta} * \delta_{A_{4}+\eta} \\
& =\sum_{\eta, \eta_{1}, \eta_{2} \in Z_{2}} q_{2}\left(\beta+\eta_{1}\right) \cdot q_{2}\left(\gamma+\eta_{2}\right) \cdot \delta_{\alpha+\delta+\eta+\eta_{1}} * \delta_{-\alpha+\delta+\eta+\eta_{2}} .
\end{aligned}
$$

Combining them again by the same formula and rearranging one finds:

$$
=\sum_{\substack{\eta \in Z_{2} \\ \zeta_{1}, \zeta_{2} \in Z_{4} \\ \zeta_{1}+\zeta_{2} \in Z_{2}}} q_{2}\left(\beta+\zeta_{1}+\zeta_{2}+\eta\right) \cdot q_{2}\left(\gamma+\zeta_{1}-\zeta_{2}+\eta\right) \cdot q_{3}\left(\alpha+\zeta_{2}\right) \cdot \delta_{\zeta_{1}+\delta} \cdot
$$

Using the relations among $q_{1}, q_{2}, q_{3}$ established in $\S 2$, this reduces to:

$$
=\sum_{\zeta \in Z_{4}} q_{1}(\beta+\gamma+2 \zeta) \cdot q_{1}(\beta-\gamma+2 \zeta) \cdot q_{1}(2 \alpha+2 \zeta) \cdot \delta_{\zeta+\delta} .
$$

With $E=\zeta+\delta$, this is exactly what we were to prove. Q.E.D.

To apply the lemma in our situation, we consider the sections of $L^{16}$, which are described by functions on $K(16 \delta)$. Let $Z_{8}$ be the group of points of order 8 in $K(16 \delta)$ (or in $K(2 \delta)$ ). For all $a \in K(16 \delta), l \in \hat{Z}_{8}$, set

$$
W_{a, l}=\sum_{\eta \in Z_{8}} l(\eta) \cdot \delta_{a+\eta} .
$$


Now suppose $A_{1}, A_{2}, A_{3}, A_{4} \in K(16 \delta)$ and $l_{1}, l_{2}, l_{3}, l_{4} \in \hat{Z}_{8} \quad$ satisfy $A_{1}+A_{2}+A_{3}+A_{4}=0, l_{1}+l_{2}+l_{3}+l_{4}=0$. We use the lemma to compute

$W_{A_{1}, l_{1}} * W_{A_{2}, l_{2}} * W_{A_{3}, l_{3}} * W_{A_{4}, l_{4}}$ $=\sum_{\substack{\eta_{1}, \eta_{2}, \eta_{3}, \eta_{4} \in Z_{8} \\ \zeta \in Z_{32} \\ 4 \zeta=\eta_{1}+\eta_{2}+\eta_{3}+\eta_{4}}} l_{1}\left(\eta_{1}\right) \cdot l_{2}\left(\eta_{2}\right) \cdot l_{3}\left(\eta_{3}\right) \cdot l_{4}\left(\eta_{4}\right) \cdot q_{4}\left(A_{1}+A_{2}+\eta_{1}+\eta_{2}-2 \zeta\right) \times$

$\times q_{4}\left(A_{1}+A_{3}+\eta_{1}+\eta_{3}-2 \zeta\right) \cdot q_{4}\left(A_{1}+A_{4}+\eta_{1}+\eta_{4}-2 \zeta\right) \cdot \delta_{\zeta}$

$=\frac{1}{8^{g}} \sum_{\eta \in \hat{Z}_{8}} \sum_{\eta_{1}, \ldots, \eta_{4} \in Z_{8}} m\left(4 \zeta-\eta_{1}-\eta_{2}-\eta_{3}-\eta_{4}\right) \cdot l_{1}\left(\eta_{1}\right) \cdot l_{2}\left(\eta_{2}\right) \times$

$\times l_{3}\left(\eta_{3}\right) \cdot l_{4}\left(\eta_{4}\right) \cdot q_{4}\left(A_{1}+A_{2}+\eta_{1}+\eta_{2}-2 \zeta\right) \times$

$\times q_{4}\left(A_{1}+A_{3}+\eta_{1}+\eta_{3}-2 \zeta\right) \cdot q_{4}\left(A_{1}+A_{4}+\eta_{1}+\eta_{4}-2 \zeta\right) \cdot \delta_{\zeta}$

where $g=\operatorname{dim} X$, (i.e., $8^{g}=\operatorname{Card}\left(Z_{8}\right)$ ). If, as usual, we let

$$
q_{4}(a, l)=\sum_{\eta \in Z_{8}} l(\eta) \cdot q_{4}(a+\eta)
$$

then this works out to be

$$
\begin{aligned}
= & \frac{1}{8^{g}} \sum_{\substack{m \in \hat{Z}_{8} \\
\zeta \in Z_{32}}}\left(\sum_{\eta_{1} \in Z_{8}} l_{1}\left(\eta_{1}\right)^{2} \cdot m\left(\eta_{1}\right)^{+2}\right) \cdot m(4 \zeta) \cdot q_{4}\left(A_{1}+A_{2}-2 \zeta, l_{2}-m\right) \times \\
& \times q_{4}\left(A_{1}+A_{3}-2 \zeta, l_{3}-m\right) \cdot q_{4}\left(A_{1}+A_{4}-2 \zeta, l_{4}-m\right) \cdot \delta_{\zeta} \\
= & \sum_{\substack{k \in 4 \\
\zeta \in \hat{Z}_{32}}} l_{1}(4 \zeta)^{-1} \cdot k(4 \zeta)^{-1} \cdot q_{4}\left(A_{1}+A_{2}-2 \zeta, l_{1}+l_{2}+k\right) \times \\
& \times q_{4}\left(A_{1}+A_{3}-2 \zeta, l_{1}+l_{3}+k\right) \cdot q_{4}\left(A_{1}+A_{4}-2 \zeta, l_{1}+l_{4}+k\right) \cdot \delta_{\zeta} .
\end{aligned}
$$

Now since the homogeneous coordinate ring of $X$ is an integral domain, it follows that for all $A$ 's and $l$ 's as above, there are elements $k \in 4 \hat{Z}_{8}$ and $\zeta \in Z_{16}$ such that

$$
\prod_{i=2}^{4} q_{4}\left(A_{1}+A_{i}+\zeta, l_{1}+l_{i}+k\right) \neq 0
$$

Now the triple $\left(A_{1}+A_{2}, A_{1}+A_{3}, A_{1}+A_{4}\right)$ is an arbitrary triple of elements $\left(B_{1}, B_{2}, B_{3}\right)$ in $K(16 \delta)$ such that $B_{1}+B_{2}+B_{3} \in K(8 \delta)$. Similarly the triple $\left(l_{1}+l_{2}, l_{1}+l_{3}, l_{1}+l_{4}\right)$ is an arbitrary triple of elements $\left(k_{1}, k_{2}, k_{3}\right)$ in $\hat{Z}_{8}$ such that $k_{1}+k_{2}+k_{3} \in 2 \hat{Z}_{8}$. Therefore, for any 
triples

$$
\begin{gathered}
\left\{\begin{array}{l}
B_{1}, B_{2}, B_{3} \in K(8 \delta) \\
k_{1}, k_{2}, k_{3} \in 2 \hat{Z}_{8}
\end{array}\right. \\
\prod_{i=1}^{3} q_{4}\left(B_{i}+\zeta, k_{i}+k\right) \neq 0
\end{gathered}
$$

for some $\zeta \in Z_{16}, k \in 4 \hat{Z}_{8}$. But by the results of $\S 3$ on the $q$ 's,

$$
q_{2}(2 B, k)=q_{4}(B, 2 * k)
$$

for any $B \in K(8 \delta), k \in \hat{\mathrm{Z}}_{4}$ [where $2 * k$ is the character $(2 * k)(x)=k(2 x)$ as before]. This proves the triple vanishing lemma. Q.E.D.

Corollary. If $X_{4} \subset H(L)$, then (b)' and (b)' are true for the homogeneous coordinate ring

$$
\bigoplus_{n=0}^{\infty} \Gamma\left(X, L^{n}\right) .
$$

In particular, if $\varphi: X \rightarrow \boldsymbol{P}_{n}$ is the embedding defined by the complete linear system $\Gamma(X, L)$, then $\varphi(X)$, as a subscheme of $\boldsymbol{P}_{n}$, is an intersection of quadric hypersurfaces.

\section{§ 5. Examples}

We shall consider 4 special cases of the preceding theory:

a) $\operatorname{dim} X=1, \delta=(2), \quad$ char $\neq 2$

b) $\operatorname{dim} X=1, \delta=(3), \quad \operatorname{char} \neq 3$

c) $\operatorname{dim} X=1, \delta=(4), \quad \operatorname{char} \neq 2$

d) $\operatorname{dim} X=2, \delta=(2,2)$, char $\neq 2$.

If $e \in X$ is the origin, then in the first 3 cases, we shall take $L$ to be $o_{X}(2 e), o_{X}(3 e)$, and $o_{X}(4 e)$, respectively. As is well known, these linear systems define maps:

a) $\varphi: X \rightarrow P_{1}$ (a double covering),

b) $\varphi: X \hookrightarrow P_{2}$ (image a non-singular cubic curve),

c) $\varphi: X \hookrightarrow P_{3}$ (image a non-singular quartic curve, which is the complete intersection of 2 quadrics),

d) $\varphi: X \rightarrow P_{3}$ ( $X$ a double covering of $\varphi X, \varphi X$ a quartic surface with 16 nodes).

The fact that in case $\mathrm{c}), \varphi(X)$ is a complete intersection of 2 quadrics is the simplest example of Theorem 2 of $\S 4$. Incidentally, note that in cases b) and c), $\oplus \Gamma\left(X, L^{n}\right)$ is actually generated by $\Gamma(X, L)$ : at least in these cases, Theorem 1 is not the best possible result. 
Case a. $H(L)=X_{2}$, and the action of the group of translations by points of $X_{2}$ extends to $\boldsymbol{P}_{1}$ so as to make $\varphi$ commute with these actions. This means that the group $Z / 2 Z \oplus Z / 2 Z$ acts on $\boldsymbol{P}_{1}$, and this action can be normalized by a suitable choice of coordinates in $\boldsymbol{P}_{1}$ so that it is given by the matrices:

$$
\left(\begin{array}{ll}
1 & 0 \\
0 & 1
\end{array}\right), \quad\left(\begin{array}{cc}
1 & 0 \\
0 & -1
\end{array}\right), \quad\left(\begin{array}{ll}
0 & 1 \\
1 & 0
\end{array}\right), \quad\left(\begin{array}{cc}
0 & 1 \\
-1 & 0
\end{array}\right) .
$$

This normalizes the map $\varphi$ among projectively equivalent maps. Since the 4 branch points must be permuted by the action of this group, they have coordinates:

$$
(\lambda,-\lambda, 1 / \lambda,-1 / \lambda) \text {. }
$$

Here $\lambda$ is the coordinate of $\varphi(e)$, and it can be anything (except for degenerate values $0, \pm 1, \pm i, \infty)$ : moreover, the abelian variety $X$ is determined by $\lambda$.

Case b. $H(L)=X_{3}$, and the action of the group of translations by points of $X_{3}$ extends to $\boldsymbol{P}_{2}$. Therefore, the group $\boldsymbol{Z} / 3 \boldsymbol{Z} \oplus \boldsymbol{Z} / 3 \boldsymbol{Z}$ acts on $\boldsymbol{P}_{2}$, and this action can be normalized by a suitable choice of coordinates in $\boldsymbol{P}_{2}$ so that it is given by the matrices:

$$
\begin{aligned}
& \left(\begin{array}{lll}
1 & 0 & 0 \\
0 & 1 & 0 \\
0 & 0 & 1
\end{array}\right), \quad\left(\begin{array}{ccc}
1 & 0 & 0 \\
0 & \omega & 0 \\
0 & 0 & \omega^{2}
\end{array}\right), \quad\left(\begin{array}{ccc}
1 & 0 & 0 \\
0 & \omega^{2} & 0 \\
0 & 0 & \omega
\end{array}\right) \\
& \left(\begin{array}{lll}
0 & 1 & 0 \\
0 & 0 & 1 \\
1 & 0 & 0
\end{array}\right), \quad\left(\begin{array}{ccc}
0 & 1 & 0 \\
0 & 0 & \omega \\
\omega^{2} & 0 & 0
\end{array}\right), \quad\left(\begin{array}{ccc}
0 & 1 & 0 \\
0 & 0 & \omega^{2} \\
\omega & 0 & 0
\end{array}\right) \\
& \left(\begin{array}{lll}
0 & 0 & 1 \\
1 & 0 & 0 \\
0 & 1 & 0
\end{array}\right), \quad\left(\begin{array}{ccc}
0 & 0 & 1 \\
\omega & 0 & 0 \\
0 & \omega^{2} & 0
\end{array}\right), \quad\left(\begin{array}{ccc}
0 & 0 & 1 \\
\omega^{2} & 0 & 0 \\
0 & \omega & 0
\end{array}\right) .
\end{aligned}
$$

The image cubic must be invariant under this group, and must be contained in the open set

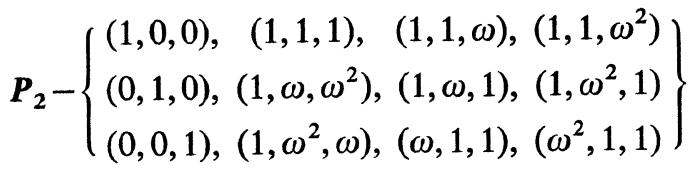

where $\boldsymbol{Z} / 3 \boldsymbol{Z} \oplus \boldsymbol{Z} / 3 \boldsymbol{Z}$ acts freely. This implies that the image cubic is given by an equation:

$$
X^{3}+Y^{3}+Z^{3}-3 \mu X Y Z, \quad \mu \neq 1, \omega, \omega^{2}, \infty .
$$


Moreover, $\varphi(e)$ must be a point of inflexion of this curve, and it turns out that the 9 points of inflexion are the 9 base points of this pencil of cubics:

$$
\begin{aligned}
& (0,1, \quad-1) \\
& (0,1,-\omega) \\
& \left(0,1,-\omega^{2}\right) \\
& (1, \quad 0, \quad-1) \\
& \left(\begin{array}{lll}
1, & 0, & -\omega) \\
(1, & 0, & -\omega^{2}
\end{array}\right) \\
& (1,-1, \quad 0) \\
& (1,-\omega, \quad 0) \\
& \left(1,-\omega^{2}, \quad 0\right) .
\end{aligned}
$$

So in this case, $\varphi(e)$ is completely determined (up to a finite choice) and its coordinates do not determine $X$. In other words, the function $q_{L}$ on $K(\delta)=Z / 3 Z$ is independent of $X$ in this case: but since $L$ is not totally symmetric, this restriction cannot come out of our theory.

Case c. $H(L)=X_{4}$, and we get, exactly as before, a group isomorphic to $\boldsymbol{Z} / 4 \boldsymbol{Z} \oplus \boldsymbol{Z} / 4 \boldsymbol{Z}$ acting on $\boldsymbol{P}_{3}$. Again, in suitable coordinates, it is the group generated by

$$
U=\left(\begin{array}{llll}
0 & 1 & 0 & 0 \\
0 & 0 & 1 & 0 \\
0 & 0 & 0 & 1 \\
1 & 0 & 0 & 0
\end{array}\right) \text { and } V=\left(\begin{array}{cccc}
1 & 0 & 0 & 0 \\
0 & i & 0 & 0 \\
0 & 0 & -1 & 0 \\
0 & 0 & 0 & -i
\end{array}\right)
$$

In this case, it will be easier to write everything down if we spell out the connection with our previous notation more precisely. First of all, the group generated by $U, V$ and scalar matrices is exactly $\mathscr{G}(\delta)$, if we let $U$ correspond to $(1 ; 1,0)$ and let $V$ correspond to $(1 ; 0, \chi)$ where $\chi \in \operatorname{Hom}\left(\boldsymbol{Z} / 4 \boldsymbol{Z}, k^{*}\right)$ is defined by $\chi(1)=i$. Moreover, the affine space $k^{4}$ on which $U, V$ act is exactly $V(\delta)$ if we let $\left(\alpha_{0}, \alpha_{1}, \alpha_{2}, \alpha_{3}\right) \in k^{4}$ correspond to the function $f$ :

$$
f(i)=\alpha_{i}
$$

[One should check that the action of $\mathscr{G}(\delta)$ on $V(\delta)$ goes over to matrix multiplication.] Regard $\boldsymbol{P}_{3}$ as the set of hyperplanes in $k^{4}$ (á la GROTHENDIECK), so that the following are isomorphic:

$$
V(\delta) \cong k^{4} \cong \Gamma\left(\boldsymbol{P}_{3}, \boldsymbol{o}(1)\right) \cong \Gamma(X, L) .
$$

Here homogeneous coordinates $X_{0}, X_{1}, X_{2}, X_{3}$ in $\boldsymbol{P}_{3}$ correspond to the points $(1,0,0,0),(0,1,0,0),(0,0,1,0)$, and $(0,0,0,1)$ in $k^{4}$ and to the 24 Invent. math., Bd. 1 
functions $\delta_{0}, \delta_{1}, \delta_{2}, \delta_{3}$ in $V(\delta)$. And the 4-tuple $\left(q_{L}(0), q_{L}(1), q_{L}(2), q_{L}(3)\right)$ is a set of homogeneous coordinates of $\varphi(e) \in \boldsymbol{P}_{3}$. To determine the quadratic equations satisfied by the coordinates $X_{i}$ on $\varphi(X)$, we refer back to the proof of Theorem $1, \S 4$, where the quadratic expressions in the functions $\delta_{a}$ were expressed in terms of a basis of $\Gamma\left(X, L^{2}\right)$ : or, more precisely, in terms of functions on $K(2 \delta) \cong Z / 8 Z$. We find

Therefore:

$$
\begin{array}{r}
\delta_{0} * \delta_{0}+\delta_{2} * \delta_{2}=\left(q_{L^{2}}(0)+q_{L^{2}}(4)\right) \cdot\left(\delta_{0}+\delta_{4}\right) \\
\delta_{0} * \delta_{1}+\delta_{2} * \delta_{3}=\left(q_{L^{2}}(1)+q_{L^{2}}(5)\right) \cdot\left(\delta_{1}+\delta_{5}\right) \\
2 \delta_{0} * \delta_{2}=\left(q_{L^{2}}(2)+q_{L^{2}}(6)\right) \cdot\left(\delta_{2}+\delta_{6}\right) \\
\delta_{0} * \delta_{3}+\delta_{2} * \delta_{1}=\left(q_{L^{2}}(3)+q_{L^{2}}(7)\right) \cdot\left(\delta_{3}+\delta_{7}\right) \\
\delta_{1} * \delta_{1}+\delta_{3} * \delta_{3}=\left(q_{L^{2}}(0)+q_{L^{2}}(4)\right) \cdot\left(\delta_{2}+\delta_{6}\right) \\
2 \delta_{1} * \delta_{3}=\left(q_{L^{2}}(2)+q_{L^{2}}(6)\right) \cdot\left(\delta_{0}+\delta_{4}\right) \\
\delta_{0} * \delta_{0}-\delta_{2} * \delta_{2}=\left(q_{L^{2}}(0)-q_{L^{2}}(4)\right) \cdot\left(\delta_{0}-\delta_{4}\right) \\
\delta_{0} * \delta_{1}-\delta_{2} * \delta_{3}=\left(q_{L^{2}}(1)-q_{L^{2}}(5)\right) \cdot\left(\delta_{1}-\delta_{5}\right) \\
\delta_{0} * \delta_{3}-\delta_{2} * \delta_{1}=\left(q_{L^{2}}(3)-q_{L^{2}}(7)\right) \cdot\left(\delta_{3}-\delta_{7}\right) \\
\delta_{1} * \delta_{1}-\delta_{3} * \delta_{3}=\left(q_{L^{2}}(0)-q_{L^{2}}(4)\right) \cdot\left(\delta_{2}-\delta_{6}\right)
\end{array}
$$

$$
\begin{aligned}
\left(q_{L^{2}}(2)+q_{L^{2}}(6)\right) \cdot\left[\delta_{0} * \delta_{0}+\delta_{2} * \delta_{2}\right] & =\left(q_{L^{2}}(0)+q_{L^{2}}(4)\right) \cdot\left[2 \delta_{1} * \delta_{3}\right], \\
\left(q_{L^{2}}(0)+q_{L^{2}}(4)\right) \cdot\left[2 \delta_{0} * \delta_{2}\right] & =\left(q_{L^{2}}(2)+q_{L^{2}}(6)\right) \cdot\left[\delta_{1} * \delta_{1}+\delta_{3} * \delta_{3}\right] .
\end{aligned}
$$

Note that $q_{L^{2}}(2)+q_{L^{2}}(6)=2 q_{L^{2}}(2)$ is not equal to 0 , or else the above equations do not define an integral domain. Set $\lambda=\left(q_{L^{2}}(0)+q_{L^{2}}(4)\right) / 2 q_{L^{2}}(2)$. Then $\varphi(X)$ is given by the 2 equations:

$$
\begin{aligned}
& X_{1}^{2}+X_{3}^{2}=2 \lambda X_{0} X_{2} \\
& X_{0}^{2}+X_{2}^{2}=2 \lambda X_{1} X_{3} .
\end{aligned}
$$

Conversely, for any $\lambda \neq 0, \pm 1, \pm i, \infty$, this defines an elliptic curve invariant under $\mathscr{G}(\delta)$. Now consider the coordinates of $\varphi(e)$ : by symmetry, $q_{L}(1)=q_{L}(3)$, i.e., $\varphi(e)$ lies on the plane $X_{1}=X_{3}$. Therefore it satisfies:

$$
\begin{aligned}
q_{L}(1)^{2} & =\lambda q_{L}(0) \cdot q_{L}(2) \\
q_{L}(0)^{2}+q_{L}(2)^{2} & =2 \lambda q_{L}(1)^{2} .
\end{aligned}
$$

Therefore, $\lambda$ can be calculated from the coordinates of $\varphi(e)$ by

$$
\lambda=\frac{q_{L}(1)^{2}}{q_{L}(0) \cdot q_{L}(2)}
$$


and the $q_{L}$ 's themselves also satisfy:

$$
q_{L}(0)^{3} \cdot q_{L}(2)+q_{L}(0) \cdot q_{L}(2)^{3}=2 \cdot q_{L}(1)^{4} .
$$

This equation is the one equation to which RiemanN's theta relations reduce in this case: it is a simple transformation of the ancient theta relation of JACOBI. Moreover, one can show that this is the only equation satisfied by the $q_{L}$ 's, aside from the inequalities:

$$
\begin{aligned}
& q_{L}(n) \neq 0, \text { any } n \\
& q_{L}(0) \neq \pm q_{L}(2) .
\end{aligned}
$$

JACOBI's theta relation comes out by using the usual basis for the vector space of theta-null values:

$$
\begin{aligned}
& \vartheta\left[\begin{array}{l}
0 \\
0
\end{array}\right]=q(0)+q(2) \\
& \vartheta\left[\begin{array}{l}
0 \\
1
\end{array}\right]=q(1)+q(3)=2 q(1) \\
& \vartheta\left[\begin{array}{l}
1 \\
0
\end{array}\right]=q(0)-q(2) \\
& \vartheta\left[\begin{array}{l}
1 \\
1
\end{array}\right]=q(1)-q(3)=0 .
\end{aligned}
$$

(Compare the comments at the end of $\S 3$.) We get:

and

$$
\vartheta\left[\begin{array}{l}
0 \\
0
\end{array}\right]^{4}=\vartheta\left[\begin{array}{l}
0 \\
1
\end{array}\right]^{4}+\vartheta\left[\begin{array}{l}
1 \\
0
\end{array}\right]^{4}
$$

$$
\vartheta\left[\begin{array}{l}
0 \\
0
\end{array}\right], \quad \vartheta\left[\begin{array}{l}
0 \\
1
\end{array}\right], \quad \vartheta\left[\begin{array}{l}
1 \\
0
\end{array}\right] \neq 0 .
$$

Case d. $H(L)=X_{2} \cong Z / 2 Z \oplus Z / 2 Z \oplus Z / 2 Z \oplus Z / 2 Z$. This group acts on the $P_{3}$ ambient to $\varphi(X)$. In suitable coordinates, it is the group $\mathscr{G}(2,2)$ generated by the matrices:

$$
\begin{aligned}
& \left(\begin{array}{llll}
0 & 1 & 0 & 0 \\
1 & 0 & 0 & 0 \\
0 & 0 & 0 & 1 \\
0 & 0 & 1 & 0
\end{array}\right), \quad\left(\begin{array}{llll}
0 & 0 & 1 & 0 \\
0 & 0 & 0 & 1 \\
1 & 0 & 0 & 0 \\
0 & 1 & 0 & 0
\end{array}\right), \\
& \left(\begin{array}{cccc}
1 & 0 & 0 & 0 \\
0 & -1 & 0 & 0 \\
0 & 0 & 1 & 0 \\
0 & 0 & 0 & -1
\end{array}\right), \quad\left(\begin{array}{cccc}
1 & 0 & 0 & 0 \\
0 & 1 & 0 & 0 \\
0 & 0 & -1 & 0 \\
0 & 0 & 0 & -1
\end{array}\right) \text {. }
\end{aligned}
$$

24* 
Then $\varphi X$ is a quartic surface, invariant under this group and containing only a finite number of points fixed under non-trivial transformations in this group. This implies that $\varphi X$ is given by the zeroes of an equation:

$$
\begin{aligned}
F(x, y, z, w)=A\left(x^{4}+y^{4}\right. & \left.+z^{4}+w^{4}\right)+B(x y z w)+C\left(x^{2} y^{2}+z^{2} w^{2}\right)+ \\
& +D\left(x^{2} w^{2}+y^{2} z^{2}\right)+E\left(x^{2} z^{2}+y^{2} w^{2}\right)=0 .
\end{aligned}
$$

These equations define a 4-dimensional family of quartics with no base points: the generic member of this family is non-singular so it could not be equal to $\varphi X$. But as soon as a surface in this family has 1 node, it acquires 16 of them: namely the images of this node under the group $\mathscr{G}(2,2)$. Such a node exists if and only if

$$
\Delta(A, B, C, D, E)=0
$$

where $\Delta$ is the discriminant of $F$. The nodes are the points $\varphi(a), a \in X_{2}$, and they may exist anywhere at all in $\boldsymbol{P}_{3}$, except on a degenerate subvariety that we will not describe. Any quartic of the above type with exactly 16 nodes and no higher singularities is of the type $\varphi X$ for some abelian surface $X$.

\section{References}

[1] BAILy, W.jr.: On the theory of $\vartheta$-functions, the moduli of abelian varieties, and the moduli of curves. Annals of Math. 75, 342-381 (1962).

[2] CARTIER, P.: Unitary representations and theta functions. Proc. of 1965 AMS Summer Institute in Boulder, to appear.

[3] Grothendieck, A.: Séminaire de géométrie algébrique. Inst. des Hautes Études Sci., 1960-61 (Mimeographed).

[4] - Séminaire: Schemas en groupes. Inst. des Hautes Études Sci., 1963-64 (Mimeographed).

[5] -, et J. DieudonNÉ: Éléments de la géométrie algébrique. Publ. de l'Inst. des Hautes Études Sci., No 4, 8, 11, ...

[6] IguSA, J.-I.: On the graded ring of theta-constants. Am. J. Math. 86, 219-246 (1964); 88, 221 - 236 (1966).

[7] LANG, S.: Abelian varieties. New York: Interscience 1958.

[8] MaCkey, G.: On a theorem of Stone and von Neumann. Duke Math. J. 16, 313-340 (1949).

[9] Mumford, D.: Geometric invariant theory. Berlin-Heidelberg-New York: Springer 1965.

[10] - Curves on an algebraic surface (to appear in Annals of Math. Studies).

[11] Siegel, C.: Moduln abelscher funktionen. Nachrichten der Akad. Göttingen, 1964.

[12] WeIL, A.: Sur certaines groupes d'operateurs unitaire. Acta Math. 111, 145-211 (1964).

\section{Department of Mathematics \\ Harvard University}

Cambridge, Massachussetts

(Received January 17, 1966) 